\title{
Linear and Nonlinear Rossby Waves in Basins both with and without a Thin Meridional Barrier by
}

Juli Atherton

B.S., McGill University (1997)

Submitted to the Joint Program in Physical Oceanography in partial fulfillment of the requirements for the degree o Master of Science
at the
MASSACHUSETTS INSTITUTE OF TECHNOLOGY
and the
WOODS HOLE OCEANOGRAPHIC INSTITUTION

MEL/WHOI LIRRARY

Woods Hole, MA Marine Eiological labofathy Woods Hole Oceanomonphte Institution

February 2002

(C) Juli Atherton, MMII. All rights reserved.

The author hereby grants to MIT and WHOI permission to reproduce and distribute publicly paper and electronic copies of this thesis document in whole or in part.

Author .........................

Joint Program in Physical Oceanography Nov 20, 2001

Certified by . .

Joseph Pedlosky

Senior Scientist

Henry L. and Grace Doherty Oceanographer Thesisy Supervisor

Accepted by

Carl Wunsch

Cecil and Ida Green Professor of Physical Oceanography Chairman, Joint Committee for Physical Oceanography 


\title{
Linear and Nonlinear Rossby Waves in Basins both with and without a Thin Meridional Barrier
}

\author{
by \\ Juli Atherton \\ Submitted to the Joint Program in Physical Oceanography \\ on Nov 20, 2001, in partial fulfillment of the \\ requirements for the degree of \\ Master of Science
}

\begin{abstract}
The linear and nonlinear Rossby wave solutions are examined in homogeneous square basins on the $\beta$-plane both with and without a thin meridional barrier. In the presence of the meridional barrier the basin is almost partitioned into two; only two small gaps of equal width, $d$, to the north and south of the barrier allow communication between the eastern. and western sub-basins.

Solutions are forced by a steady periodic wind forcing applied over a meridional strip near the eastern side. Bottom friction is present to allow the solutions to reach equilibrium. The linear solution for the basin containing the barrier is determined analytically and the nonlinear solutions for both basins are found numerically.

In the linear solution with the barrier present, particular attention was paid to the resonant solutions. We examined the effects of varying the symmetry of the forcing about the mid-latitude, the frequency of the periodic forcing and the strength of the bottom friction. For each solution we focus on how the no net circulation condition, which is central to any solution in a barrier basin, is satisfied.

The nonlinear solutions were studied for both basin configurations. In each case the transition from the weakly nonlinear solution to the turbulent solution was examined, as the forcing frequency and forcing strength were varied. Only integer multiples of the forcing frequency are present in the weakly nonlinear solutions. The turbulent solutions were accompanied by the appearance of many other frequencies whose exact origins are unknown, but are probably the result of instabilities.

A hysteresis was found for the turbulent solutions of both the barrier-free and barrier basins.

In the weakly nonlinear solutions of the barrier basin it was predicted and confirmed that there is never a steady net flow from sub-basin to sub-basin. It was also shown that with a symmetric forcing all modes oscillating with an odd multiple of the forcing frequency are symmetric and all modes oscillating with even multiples of the forcing frequency are antisymmetric.
\end{abstract}


Thesis Supervisor: Joseph Pedlosky

Title: Senior Scientist

Henry L. and Grace Doherty Oceanographer 


\section{Acknowledgments}

I would like to thank my advisor, Joe Pedlosky, for introducing me to the the problem of studying Rossby modes for various basin configurations and for helping me publish my pregenerals research (presented in chapter 3 of this thesis) in Il Nuovo Cimento. I am especially grateful for the way Joe has made himself readily available for discussions and for his promptness in returning my work and providing feedback.

I would also like to thank my committee members Mike Spall, Joe LaCasce, Nelson Hogg, and Glenn Flierl. In particular, I thank Mike Spall for giving me.his modified version of MICOM when I began my research.

Special thanks are due to thank my classmate Pablo Zurita who has been a good friend and has helped me immensely in the last few years. Through numerous discussions we have had, I have learnt a great deal me much about computers and numerical computation. This not only helped me to get MICOM running, but has provided me with skills that will be very helpful in the future. I am also grateful to Pablo for allowing me to run MICOM and store my results on his computer.

I am indebted to Brian Arbic who directed the computer Huron my way when he went to Princeton. Huron has been a great benefit to me this last year. I would alsolike to thank Richard Wardle, Francis Poulin, and Samar Khatiwala for interesting discussions regarding this thesis.

Last, but not least, I would like to thank my parents, sister, and Adrian for all their encouragement. 


\section{Contents}

1 Introduction $\quad 15$

2 Basin Modes $\quad 18$

2.1 Barrier-Free Basin . . . . . . . . . . . . . . . . . . . . . 19

2.2 Barrier Basin - Containing Meridional Barrier . . . . . . . . . 20

2.3 Inner Product of Modes . . . . . . . . . . . . . . . . . . 22

2.4 Projection of Solution Onto the Basin Modes . . . . . . . . . . 23

3 Linear Barrier Basin Problem $\quad 25$

3.1 Analytical Solution . . . . . . . . . . . . . . . 25

3.2 Results . . . . . . . . . . . . . . . . . . . . . . . . 31

3.2 .1 Symmetric Forcing . . . . . . . . . . . . . . . . 32

3.2 .2 Antisymmetric Forcing . . . . . . . . . . . . . . . 34

3.2 .3 Asymmetric Forcing . . . . . . . . . . . . . . . . 34

$3.2 .4 \quad$ Bottom Friction . . . . . . . . . . . . . . . . . . . . . 35

3.3 Analysis - Another Look at the QG Equation ... . . . . . 37

4 Numerical Model $\quad 39$

4.1 MICOM Model . . . . . . . . . . . . . . . . . . . . . 39

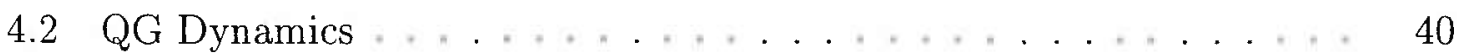

4.3 Numerical Stability and Lateral Friction . . . . . . . . . . . . . 43

4.4 Scaling for the Numerical Runs . . . . . . . . . . . . . . 44 
5 Nonlinear Solution for the Barrier-Free Basin

5.1 Previous Weakly Nonlinear Results for the Periodically Forced Barrier Basin . . . . . . . . . . . . . . . . . . . . . . 4 48

5.1.1 Bottom Friction and Forcing Larger than the Nonlinearity . . 49

5.1.2 Nonlinearity Larger than the Bottom Friction and Forcing . . 50

5.2 Numerical Results. . . . . . . . . . . . . . . . . . . . . 51

5.3 Description of Results . . . . . . . . . . . . . . . 53

5.4 Analysis of Results . . . . . . . . . . . . . . . 56

6 Nonlinear Solution for the Barrier Basin 62

6.1 Numerical Results . . . . . . . . . . . . . . . . . . . . . . 62

6.2 Description of Results . . . . . . . . . . . . . . 62

6.3 Analysis of Results . . . . . . . . . . . . . . . . 66

7 Conclusions $\quad 72$

$\begin{array}{ll}\text { A Tables } & 76\end{array}$

$\begin{array}{ll}\text { B Figures } & 78\end{array}$

C Analytical Expression for Variance in the Linear Barrier Basin Problem 


\section{List of Figures}

B-1 Sketch of the square barrier basin with the meridional barrier placed at $x_{I}$ and the forcing along the dotted line at $x_{F}$. There are two gaps of width $d$ to the north and south of the barrier.

B-2 Semilog plots of $\left|\phi_{I}\right|$ (- - ) and (a) full basin variance, (b) eastern subbasin variance and (c) western sub-basin variance vs forcing frequency $\omega_{F_{n . d .}}$ for the case where $x_{I}=0.3, x_{F}=0.7, d=0.05, \gamma=10^{-8}$ and the meridional structure of the forcing is symmetric about $y=0.5$.

B-3 Contour plots of the absolute value of the envelope function, $|\phi(x, y)|$, for the case where $x_{I}=0.3, x_{F}=0.7, d=0.05, \gamma=10^{-8}$, and the meridional structure of the forcing is symmetric about $y=0.5$. The forcing frequencies, shown under each plot, correspond to the first 7 peaks in the full basin variance graph in Figure B-2 (a). The value EC shown to the left of each forcing frequency is the circulation on the eastern side of the barrier (see appendix $\mathrm{C}$ for calculation of $\mathrm{EC}$ ) ...

B-4 Semilog plots of (a) full basin variance, (b) eastern sub-basin variance vs forcing frequency $\omega_{F_{n . d .}}$. Plots (c) and (d) are contours of the absolute value of the envelope function, $|\phi(x, y)|$, at frequencies corresponding to the first two peaks of the full variance. $x_{I}=0.3$, $x_{F}=0.7, d=0.05, \gamma=10^{-8}$ and antisymmetric meridional structure of the forcing about $y=0.5 \ldots \ldots \ldots \ldots \ldots$ 
B-5 Semilog plots of $\left|\phi_{I}\right|$ (- - ) and (a) full basin variance, (b) eastern subbasin variance and (c) western sub-basin variance vs forcing frequency $\omega_{F_{n . d .} .}$ for $x_{I}=0.3, x_{F}=0.7, d=0.05, \gamma=10^{-8}$, and the asymmetric meridional structure of the forcing. . . . . . . . . . . .

B-6 Contour plots for $x_{I}=0.3, x_{F}=0.7, d=0.05, \gamma=10^{-8}$, and the asymmetric meridional structure of the forcing. The forcing frequencies, under each plot, correspond to the first nine peaks in the full basin variance graph in Figure 5 (a). The value EC shown next to the forcing frequency is the circulation on the east side of the barrier (see appendix $\mathrm{C}$ for calculation of $\mathrm{EC}) . \ldots \ldots \ldots$

B-7 Semilog plots of $\left|\phi_{I}\right|$ (- - ) and full basin variance vs forcing frequency $\omega_{F_{n . d .}}$ for $x_{I}=0.3, x_{F}=0.7, d=0.05$, and asymmetric meridional structure of the forcing, and varying friction (a) $\gamma=0.0001$, (b) $\gamma=$ 0.001, (c) $\gamma=0.0025$, (d) $\gamma=0.005$, (e) $\gamma=0.01$, (f) $\gamma=0.0167$.

B-8 The (a) full basin, (b) eastern sub-basin and (c) western sub-basin variance response vs the bottom friction parameter $\gamma$ for $\omega_{F n, d,}=0.08$ for the barrier basin analytical solution. . . . .

B-9 Contours of the envelope (i.e. absolute value $|\phi(x, y)|$ ) for $\omega_{F_{n . d .}}=0.08$ and certain values of the bottom friction parameter $\gamma$ in the linear barrier basin solution. (a) $\gamma=0.005$, (b) $\gamma=0.01$, (c) $\gamma=0.015$, (d) $\gamma=0.02$

B-10 Amplitude response curve calculated using the method presented in [3] The parameters $k=0, m=2, n=1, f=1$, and, $\delta=0.126$ correspond to our first numerical run where $\left(\frac{\delta_{I}}{L}\right)_{W N}^{2}=0.002$.

B-11 Mean integrated kinetic energy vs forcing frequency, $\omega_{F_{n . d .}}$, for the series of numerical runs with increasing nonlinearity. Notice how the peaks lean towards higher frequencies and then ultimately disintegrate. Also, note the transition in the solution occurring at the nondimensional frequencies $\omega_{F_{n . d .}}, 0.0775,0.08,0.0875$, and 0.085 for the wind forcing strengths $\left(\frac{\delta_{I}}{L}\right)_{W N}^{2}=0.004,0.006,0.008$, and 0.01 , respectively. 
B-12 Envelopes calculated over one forcing period demonstrating that the weakly nonlinear solution is dominated by the $(2,1)$ mode just before the transition which occurs when the forcing frequency is decreased. (a) $\left(\frac{\delta_{I}}{L}\right)_{W N}^{2}=0.004$ and $\omega_{F_{n . d .}}=0.0775$, (b) $\left(\frac{\delta_{I}}{L}\right)_{W N}^{2}=0.006$ and $\omega_{F_{n . d .}}=0.0825,(\mathrm{c})\left(\frac{\delta_{I}}{L}\right)_{W N}^{2}=0.008$ and $\omega_{F_{n . d .}}=0.085$, and $(\mathrm{d})$ $\left(\frac{\delta_{r}}{L}\right)_{W N}^{2}=0.01$ and $\omega_{F_{n . d .}}=0.0875$.

B-13 Envelope plots taken over one forcing period showing the solution just after the transition to the very nonlinear solution when the forcing frequency is decreased.(a) $\left(\frac{\delta_{I}}{L}\right)_{W N}^{2}=0.004$ and $\omega_{F_{n . d .}}=0.0775$, (b) $\left(\frac{\delta_{I}}{L}\right)_{W N}^{2}=0.006$ and $\omega_{F_{n . d .}}=0.0825$, (c) $\left(\frac{\delta_{I}}{L}\right)_{W N}^{2}=0.008$ and $\omega_{F_{n . d .}}=$ 0.085 , and $(\mathrm{d})\left(\frac{\delta_{r}}{L}\right)_{W N}^{2}=0.01$ and $\omega_{F_{n . d .}}=0.0875 \ldots \ldots$

B-14 Contours of the stream function, $\Psi$, at 2 day intervals for the solution $\left(\frac{\delta_{I}}{L}\right)_{W N}^{2}=0.004$ and $\omega_{F n \text {.d. }}=0.0725$. The contours span 24 days of the 25.08 day forcing period. Solid lines correspond to positive stream lines and dotted lines correspond to negative streamlines. The contours document the westward propagation of the solution during one forcing period. Note $N L$ in the figure denotes $\left(\frac{\delta_{I}}{L}\right)_{W N}^{2}$.

B-15 Contours of the stream function, $\Psi$, at 2 day intervals for the solution $\left(\frac{\delta_{I}}{L}\right)_{W N}^{2}=0.01$ and $\omega_{F n . d .}=0.0725$. The contours span 24 days of the 25.08 day forcing period. Solid lines correspond to positive stream lines and dotted lines correspond to negative streamlines. The contours document the westward propagation of the solution during one forcing period. Note $N L$ in the figure denotes $\left(\frac{\delta_{I}}{L}\right)_{W N}^{2} \ldots \ldots \ldots$

B-16 The maximum zonal and meridional velocities plotted vs the nondimensional forcing frequencies, $\omega_{F_{n . d .}}$, for each set of nonlinear runs. Note $N L$ in the figure denotes $\left(\frac{\delta_{I}}{L}\right)_{W N}^{2} \ldots \ldots \ldots \ldots$

B-17 Fourier transform of the zonal velocity at the point $(800 \mathrm{~km}, 800 \mathrm{~km})$ for the nonlinear solutions with a forcing frequency of $\omega_{F n \text {.d. }}=0.0725$. Note $N L$ in the figure denotes $\left(\frac{\delta_{I}}{L}\right)_{W N}^{2} \ldots \ldots \ldots$ 
B-18 Contour plots of the absolute values of the spatial coefficients of the terms in a Fourier time series which oscillate at frequencies that are integral multiples of the forcing frequencies. Note $N L$ in the figure denotes $\left(\frac{\delta_{I}}{L}\right)_{W N}^{2}$

B-19 Fourier transform of the zonal velocity for different points in the basin at mid-latitude over 1200 days (a) east side, (b) middle, (c) west side. $\left(\frac{\delta_{r}}{L}\right)_{W N}^{2}=0.004$ and $\omega_{F n . d .}=0.0725$, note higher frequencies appearing as we proceed west. . . . . . . . . . . .

B-20 Finite difference calculation of the model output for the terms in the QG equation excluding the forcing term. $\left(\frac{\delta_{I}}{L}\right)_{W N}^{2}=0.008$ and $\omega_{F n . d .}=$ 0.085. This is just before the solution transitions to the turbulent regime at $\omega_{F n \text {.d. }}=0.0825$. Note $N L$ in the figure denotes $\left(\frac{\delta_{I}}{L}\right)_{W N}^{2}$. .

B-21 Finite difference calculation of the model output for the terms in the QG equation excluding the forcing term. The solution has a wind forcing strength $\left(\frac{\delta_{I}}{L}\right)_{W N}^{2}=0.008$ and a forcing frequency $\omega_{F n . d .}=$ 0.0825 . This is just after the transition to the turbulent regime. Note $N L$ in the figure denotes $\left(\frac{\delta_{I}}{L}\right)_{W N}^{2} \ldots \ldots \ldots \ldots \ldots$

B-22 Mean integrated kinetic energy vs nondimensional forcing frequency, $\omega_{F_{n . d .}}$. The results plotted by squares indicates that the solution was initialized with zero initial conditions. The results plotted with the asterisk indicate that each run was initialized with the results of the previous run forced at the lower forcing frequency. A hysteresis is observed for the forcing frequencies $\omega_{F_{n . d .}}=0.085$ for zero initial conditions and $\omega_{F_{n . d .}}=0.09$ for initial conditions of lower frequency run.

B-23 (a) is the RMS of the stream function just before the transition and (b) is the RMS of the stream function just after the transition when the solution is in its weakly nonlinear state. These runs have been initialized with solutions forced at a lower frequency. Note $N L$ in the figure denotes $\left(\frac{\delta_{I}}{L}\right)_{W N}^{2}$. 
B-24 Mean integrated kinetic energy over the area of the barrier basin vs forcing frequency for runs with forcing strengths of $\left(\frac{\delta_{I}}{L}\right)_{W N}^{2}=0.004$ and 0.01 respectively. All runs were done with zero initial conditions.

B-25 The contours of the envelope function for all the solutions forced with a strength $\left(\frac{\delta_{I}}{L}\right)_{W N}^{2}=0.004$ and zero initial conditions. . . . . . . 103

B-26 The contours of the envelope function for all the solutions forced with a strength $\left(\frac{\delta_{I}}{L}\right)_{W N}^{2}=0.01$ and zero initial conditions. Note the slight change in the nonlinearity of the solution in the eastern basin between the frequencies $\omega_{F_{n . d .}}=0.075$ and $\omega_{F_{n . d .}}=0.0775$. The solution forced with a frequency of $\omega_{F_{n . d .}}=0.0775$ looks more linear than the the solution forced at $\omega_{F_{n . d .}}=0.075$.

B-27 Contours of the stream function, $\Psi$, at 2 days intervals for the solution $\left(\frac{\delta_{I}}{L}\right)_{W N}^{2}=0.004$ and $\omega_{F_{n . d .}}=0.0725$ in the barrier basin. The contours span 24 days of the 25.08 day forcing period. Solid lines correspond to positive stream lines and dotted lines correspond to negative stream lines. The contours document the westward propagation of the solution during one forcing period. . . . . . . . . . . . . . . . . 105

B-28 Contours of the stream function, $\Psi$, at 2 days intervals for the solution $\left(\frac{\delta_{I}}{L}\right)_{W N}^{2}=0.01$ and $\omega_{F_{n . d .}}=0.0725$ in the barrier basin. The contours span 24 days of the 25.08 day forcing period. Solid lines correspond to positive stream lines and dotted lines correspond to negative stream lines. The contours document the westward propagation of the solution during one forcing period. . . . . . . . . . . 106

B-29 Contour plots of $\Psi$ at two day intervals for the solution $\left(\frac{\delta_{I}}{L}\right)_{W N}^{2}=0.01$ and $\omega_{F_{n . d .}}=0.0725$. The barrier is placed at $1420 \mathrm{~km}$ to the east and the wind forcing is applied along a meridional strip from $200 \mathrm{~km}$ to $400 \mathrm{~km}$. 
B-30 Fourier transform of the zonal velocity at points along the mid-latitude. (a), (c), and (e) are the solutions for $\left(\frac{\delta_{l}}{L}\right)_{W N}^{2}=0.01$ and $\omega_{F_{n . d .}}=$ 0.0725 , while (b), (d), and (f) are the solutions for $\left(\frac{\delta_{I}}{L}\right)_{W N}^{2}=0.004$ and $\omega_{F_{n . d .}}=0.0725$. (a) and (b) are the Fourier transforms of velocities in the west of the basin, (c) and (d) are Fourier transforms in the middle, and (e) and (f) are Fourier transforms in the west of the basin.

B-31 Fourier transforms of the zonal velocity for solutions forced at 0.0725 and increasing wind strength. Figures (a), (b) and (c) indicate that as the forcing strength increases the first non-integer multiple of the forcing frequency to appear is at about 0.1 which is close to $\frac{3}{2}$ times $\omega_{F_{n . d} \text {. }}$ or the natural frequency of the first resonant mode in the barrier basin.

B-32 Envelope plot for the spatial coefficients associated with the peaks labeled 1,2,3,4,5, and 6 in Figure B-30 (a) corresponding to the solution $\left(\frac{\delta_{r}}{L}\right)_{W N}^{2}=0.004$ and $\omega_{F_{n . d .}}=0.0725 \ldots \ldots \ldots \ldots$ B-33 (a) plots the Fourier transform of the zonal velocity at a point. The peak labels, 0, 2, 4, and 6, correspond to the labels in Figure B-30 (b). (b), (c), (d), and (e) above are the absolute values of the spatial coefficients corresponding to peaks $0,2,4$, and 6 labeled in (a). . . 111

B-34 (a) plots the Fourier transform of the zonal velocity at a point. The peak are labeled correspond their relation to the forcing frequency, $\omega_{F_{n . d .}}$ (b), (c), and (d) above are the absolute values of the spatial coefficients corresponding to peaks labeled in (a). . . . . . . .

B-35 Mean integrated kinetic energy for the solutions forced with a strength of $\left(\frac{\delta_{I}}{L}\right)_{W N}^{2}=0.01$ in the barrier basin. The six pointed star represents solutions initialized with zero initial conditions and the circle represents the solution forced at $\omega_{F_{n . d .}}=0.0775$ initialized with the solution of $\omega_{F_{n . d .}}=0.075$. 
B-36 The asterisks, ${ }^{*}$, represent the mean integrated kinetic energy of the solution initialized with zero initial conditions for a wind forcing of $\left(\frac{\delta_{I}}{L}\right)_{W N}^{2}=0.01$ and bottom friction $\frac{\delta_{S}}{L}=0.001$. The circles, (o), represent the mean integrated kinetic energy of the solution forced with a nondimensional frequency of $\omega_{F_{n . d .}}=0.0775$ and initialized with the solution which was forced at $\omega_{F_{n . d .}}=0.075 . \ldots \ldots \ldots 114$

B-37 The dotted line plots the mean integrated kinetic energy vs the time in model days of the run forced with $\omega_{F_{n . d .}}=0.0775,\left(\frac{\delta_{I}}{L}\right)_{W N}^{2}=0.01$ and $\frac{\delta_{S}}{L}=0.001$ and initialized with zero initial conditions. The solid line represents the corresponding run initialized with the solution from the lower forcing frequency $\omega_{F_{n . d .}}=0.075$.

B-38 Contour plots of envelope for solutions forced at $\left(\frac{\delta_{I}}{L}\right)_{W N}^{2}=0.01, \frac{\delta_{S}}{L}=$ 0.001 , and $\omega_{F_{n . d .}}=0.0775$. (a) initialized with zero initial conditions and (b) initialized with the solution forced at $\omega_{F_{n . d .}}=0.075$. 


\section{List of Tables}

A.1 Comparison of the nine resonant solutions in Figures B-5 and B-6 to the normal modes for a barrier-free basin to which they resemble. The resemblance is observed both in the structure of the solution and in the frequencies. For the case where the dominant response is in a sub-basin we consider a basin the same size. The quotation marks indicate that a sub-basin solution is symmetric and hence is part of the subset of full basin modes and not a part of the set of antisymmetric sub-basin modes. . . . . . . . . . . 


\section{Chapter 1}

\section{Introduction}

In this thesis we consider the propagation of barotropic Rossby waves past a thin meridional barrier in a square basin on a $\beta$-plane, see Figure B-1. The barrier nearly divides the basin into two, leaving only two small gaps, of width $d$, between the north and south tips of the barrier and the basin walls. The barrier is meant to represent anything in the ocean that is steep enough to impede the propagation of Rossby waves, including continents, islands, island chains, and ridge systems. The small gaps represent spaces between these topographies, such as fracture zones in the ridge systems.

By studying the Rossby modes present in this simplified basin, we gain insight into how the Rossby waves might propagate past the topographic features mentioned above. Also, as more is learned about this simple model, extra complexities, such as a baroclinic fluid and varying topography, can be added that more closely mimic the situation in the real ocean. Therefore, we can consider these simpler situations, which are sometimes difficult to justify, as a stepping stones to understanding the more complicated but more relevant models. It is interesting to note that a variation of this model, see Pedlosky [7], has offered insight to the apparent amplification of the first vertical baroclinic mode in the Pacific west of the ridge system. See Chelton and Schlax [10] for further details.

This basin model was first introduced by Pedlosky and Spall [4] in order to study Rossby wave propagation. They had been studying the steady circulation in such 
a basin, Pedlosky et al [8], to confirm Godfrey's Island rule, Godfrey [9], in the presence of nonlinearities. At one point in their investigations they studied a twolayer baroclinic fluid that became unstable and developed eddies in the eastern basin. Surprisingly, the eddies excited Rossby modes throughout the basin. This was particularly noticeable in layer two of the model. Hence, their first work, Pedlosky and Spall [4], on the subject of Rossby waves propagating past a meridional barrier focused on identifying the Rossby modes present in such a basin. To complete this goal, they solved the QG equation containing only the time-dependent relative vorticity term and the $\beta$-term for a barotropic fluid in such a basin. They found that the modes were composed of a set of full basin modes that were symmetric about the mid-latitude and a set of eastern sub-basin modes, as well as a set of western sub-basin modes that were both antisymmetric about the mid-latitude. These modes are presented in chapter 2 for reference and for use in the analysis of the results.

Early work I conducted considered the linear, homogeneous, periodically forced solution of the basin in Figure B-1 in the presence of bottom friction. Particular attention was paid to the resonant solutions and the effects of varying the meridional structure of the forcing and the strength of the bottom friction. The resemblance of the barrier basin mode to the barrier-free basin modes was investigated. This solution is presented in Atherton [1] and in chapter 3. Also included is an analysis of the solution in terms of the island modes given in Pedlosky and Spall [4].

Chapter 2 reviews the barrier-free basin modes presented in [5] and the barrier basin modes presented in [4]. The orthogonality and inner product for both sets of Rossby modes is derived and finally, in section 2.4, there is a short discussion of how the full solution projects onto the basin modes.

The MICOM model used for the calculation of the nonlinear solutions is presented in section 4.1. Section 4.2 discusses over what parameter range this shallow water model approximates the QG dynamics. The scaling method for the parameters used in the model is given in section 4.4 .

The nonlinear barrier-free basin solution is presented in chapter 5. Sections 5.1.1 and 5.1.2 give a brief discussion of the weakly nonlinear solution for this barrier-free 
basin. The nonlinear results are examined as the solutions enter turbulent regime. Close attention is paid to the frequencies which appear in this turbulent solution. A hysteresis is also observed for the turbulent solution.

Finally, in chapter 6 both the weakly nonlinear and turbulent barrier basin solutions are studied. An antisymmetric forcing for the weakly nonlinear solution is only capable of exciting the eastern basin modes. Interestingly, for a symmetric forcing about the mid-latitude the full basin modes only oscillate at the forcing frequency and at even multiples of the forcing frequency whilst the antisymmetric modes only oscillate at odd multiples of the forcing frequency. Hence, no matter what the meridional structure of the forcing is, the steady solution is always associated to the antisymmetric modes and therefore there is never any net circulation between the sub-basins.

Chapter 7 summarizes the results presented in this thesis. 


\section{Chapter 2}

\section{Basin Modes}

To begin, we will review and describe the basin modes for both the barrier-free basin (containing no barrier) and the barrier basin (containing a meridional barrier). We will then determine the inner product for which the spatial components of the modes are orthogonal and express the solution as a summation of eigenmodes.

The modes are found by solving the nondimensional $Q G$ equation with a rigid lid without friction or forcing, see Pedlosky [5],

$$
\nabla^{2} \Psi_{t}+\Psi_{x}=0
$$

for both basin configurations with no normal flow boundary conditions. The spatial coordinates are nondimensionalized by $L$, the length of the basin, and the time is nondimensionalized by $\beta L$. In this thesis $\Psi$ will denote the nondimensional stream function and $\psi$ will denote the dimensional steam function.

The solutions can be found in Pedlosky [5] for the barrier-free basin, and Pedlosky and Spall [4] for the barrier basin. We will present and discuss these solutions in the next four sections for future reference. 


\subsection{Barrier-Free Basin}

As mentioned above, the modes in the absence of the barrier are presented in Pedlosky [5]. The problem is separable in the time, the meridional, and the zonal coordinates $t$, $x$, and $y$. Each mode has a time-dependent steady periodic component $e^{i \omega_{m n} t}$, where $\omega_{m n}$ is the eigenfrequency nondimensionalized by $\beta L$. The eigenfrequency, $\omega_{m n}$, is equal to $\frac{1}{2 \pi \sqrt{m^{2}+n^{2}}}$ where $m, n=1,2,3 \ldots$ The spatial structure of the modes is

$$
\Phi_{m n}=e^{i k_{m n} x} \sin m \pi x \sin n \pi y
$$

The full solution corresponding to the eigenfrequency $\omega_{m n}$ is

$$
\Psi_{m n}=e^{i\left(\omega_{m n} t+k_{m n} x\right)} \sin m \pi x \sin n \pi y
$$

and is composed of a westward traveling wave, $e^{i\left(\omega_{m n} t+k_{m n} x\right)}$, along with an envelope function, $\sin m \pi x \sin n \pi y$. The envelope, $\phi$, is composed of cells, the number of which are determined by the integers $m$ and $n$ and satisfies the boundary conditions of no normal flow.

Modes for which $\omega_{m n}=\omega_{p q}$ are degenerate; two different eigenmodes, $\Psi_{m n}$ and $\Psi_{p q}$, with different spatial structures, $\Phi_{m n}$ and $\Phi_{p q}$, can correspond to the same eigenfrequencies. For our square basin this will occur when $m^{2}+n^{2}=p^{2}+q^{2}$. A basin with sides $L_{x}$ and $L_{y}$ such that $\frac{L_{x}}{L_{y}}$ is not rational will lack this degeneracy.

Throughout, we will denote the spatial structure of the mode by a capital $\Phi$ and the envelope of the mode by a small $\phi$. The spatial structure excludes the time dependent part of the mode and the envelope excludes the westward wave component. For the barrier-free basin modes the difference between the spatial component, $\Phi$, and the envelope component, $\phi$, is that the spatial component has an extra factor of $e^{i k_{m n} x}$. 


\subsection{Barrier Basin - Containing Meridional Barrier}

The basin modes in the presence of the barrier are given in Pedlosky and Spall [4]. As with the barrier-free basin, these eigenmodes are determined by solving the QG equation (2.1) with no normal flow conditions in the multiply connected domain of the barrier basin. The no normal flow condition is enforced by setting the spatial component of the stream function to be zero on the basin walls and the stream function to be $\Psi_{I}=e^{i \omega t} \phi_{I}$ on the barrier, where $\phi_{I}$ is a constant (not to be confused with the envelope function $\phi(x, y))$ and where $\omega$ is the forcing frequency. For this basin configuration with a barrier there can be either full basin or sub-basin modes. The circulation constraint of no circulation about the barrier, which is derived by integrating the tangential component of the momentum equation about its perimeter, is used as another boundary condition and the flow through the gaps is assumed to be uniform across the gap. The circulation constraint will be derived in detail in section 3.1 where we solve for the linear, periodically forced, solution with the presence of bottom friction in the barrier basin.

The envelope function, $\phi$, for the full basin, western sub-basin and eastern subbasin modes is presented below. Note that $x_{I}$ is the zonal position of the barrier.

Full Basin Modes

$$
\begin{aligned}
\phi_{W E S T}(x, y) & =\sum_{n=0}^{\infty} B_{n} \frac{\sin \alpha_{n} x}{\sin \alpha_{n} x_{I}} \sin (n \pi y) \\
\phi_{E A S T}(x, y) & =\sum_{n=0}^{\infty} A_{n} \frac{\sin \left(\alpha_{n}(x-1)\right)}{\sin \left(\alpha_{n}\left(x_{I}-1\right)\right)} \sin (n \pi y)
\end{aligned}
$$

where

$$
\begin{aligned}
\alpha_{n} & =\sqrt{k^{2}-n^{2} \pi^{2}} \\
A_{n}=B_{n} & =-2 \phi_{I}\left((-1)^{n}-1\right)^{2} \frac{\sin (n \pi d)}{n^{2} \pi^{2} d}
\end{aligned}
$$

The coefficients $A_{n}$ and $B_{n}$ are determined using the assumptions that the stream function is linear in the gaps and equal to $\phi_{I}$ on the barrier. Note that $A_{n}$ and $B_{n}$ are 
non-zero only when the mode is symmetric about the mid-latitude (when $n$ is odd), and hence antisymmetric full basin modes do not exist when the gaps are placed symmetrically about the mid-latitude of the basin. The wavenumber $k$, in equation (2.6), is equal to $\frac{1}{2 \omega}$ and is determined from the following dispersion relation (2.8). Equation (2.8) is derived from the circulation constraint.

$$
\Phi_{I} \sum_{n=1}\left((-1)^{n}-1\right)^{2} \frac{\sin (2 n \pi d)}{n^{3} \pi^{3} d} \frac{\alpha_{n} \sin \alpha_{n}}{\sin \alpha_{n} x_{I} \sin \alpha_{n}\left(x_{I}-1\right)}=0
$$

It is interesting to note that the barrier basin modes and the barrier-free basin modes are often similar in structure and eigenfrequency. See Pedlosky and Spall [4], and Table A.1, for details.

The sub-basin modes are equivalent to barrier-free basin modes for a basin equal in size to the sub-basin with the stipulation that, due to the circulation condition, only sub-basin modes which are antisymmetric about the mid-latitude can exist. Hence we have the following solutions for the eastern and western sub-basin modes.

\section{Eastern Sub-Basin Modes}

$$
\begin{aligned}
\phi_{W E S T} & =0 \\
\phi_{E A S T} & =\sin \left(m \pi\left(\frac{x-1}{x_{I}-1}\right)\right) \sin (n \pi y) \\
\omega_{m n} & =\frac{1}{2 \pi \sqrt{n^{2}+\left(\frac{m}{x_{I}-1}\right)^{2}}} . \quad n=2,4,6, \ldots ; m=1,2,3, \ldots
\end{aligned}
$$

\section{Western Sub-Basin Modes}

$$
\begin{aligned}
\phi_{W E S T} & =\sin \left(m \pi \frac{x}{x_{I}}\right) \sin (n \pi y) \\
\phi_{E A S T} & =0 \\
\omega_{m n} & =\frac{1}{2 \pi \sqrt{n^{2}+\left(\frac{m}{x_{I}}\right)^{2}}} \quad n=2,4,6, \ldots ; m=1,2,3, \ldots
\end{aligned}
$$

To get the complete solutions for the full basin, western sub-basin and eastern subbasin modes we multiply the envelope functions above by the westward traveling wave 
component $e^{i\left(\omega t+k\left(x-x_{I}\right)\right)}$. Note that, because of the way the solution is constructed, the spatial structure, $\Phi$, and the envelope function, $\phi$, are equal to the island constant $\phi_{I}$ along the meridional barrier at $x_{I}$.

\subsection{Inner Product of Modes}

Following Pedlosky [5, pages 152-3], we determine the inner product for which these complete sets of modes are orthogonal. We let $\Psi_{m n}$ and $\Psi_{p q}$ denote either two barrierfree basin modes or two barrier basin modes. The form of these modes, as we have discussed in the previous two sections, is a time dependent component $e^{i \omega t}$ multiplied by the spatial component $\Phi$. Hence, substituting $\Psi_{m n}$ and $\Psi_{p q}$ into (2.1) and performing the time derivative and cancelling $e^{i \omega t}$, we obtain two equations for the spatial functions $\Phi_{m n}$ and $\Phi_{p q}$, where we have taken the complex conjugate of equation (2.10).

$$
\begin{aligned}
\omega_{m n} \nabla^{2} \Phi_{m n}-i \frac{\partial \Phi_{m n}}{\partial x} & =0 \\
\omega_{p q} \nabla^{2} \Phi_{p q}^{*}+i \frac{\partial \Phi_{p q}^{*}}{\partial x} & =0
\end{aligned}
$$

Multiplying equation (2.9) by $\Phi_{p q}^{*}$ and equation (2.10) by $\Phi_{m n}$ then subtracting, we obtain

$$
\omega_{m n} \Phi_{p q}^{*} \nabla^{2} \Phi_{m n}-\omega_{p q} \Phi_{m n} \nabla^{2} \Phi_{p q}^{*}-i \Phi_{p q}^{*} \frac{\partial \Phi_{m n}}{\partial x}-i \Phi_{m n} \frac{\partial \Phi_{p q}^{*}}{\partial x}=0
$$

Using the vector relationship $F \nabla^{2} G=\vec{\nabla} F \cdot \vec{\nabla} G-\vec{\nabla} \cdot(F \vec{\nabla} G)$ on the first two terms and the product rule on the third and fourth term, we obtain the following

$$
\left(\omega_{m n}-\omega_{p q}\right) \vec{\nabla} \Phi_{p q}^{*} \cdot \vec{\nabla} \Phi_{m n}-\omega_{m n} \vec{\nabla} \cdot\left(\Phi_{p q}^{*} \vec{\nabla} \Phi_{m n}\right)+\omega_{p q} \vec{\nabla} \cdot\left(\Phi_{m n} \vec{\nabla} \Phi_{p q}^{*}\right)-i \frac{\partial\left(\Phi_{p q}^{*} \Phi_{m n}\right)}{\partial x}=0
$$

Next we integrate equation (2.12) over the area of the basin $\mathrm{D}$, excluding the barrier if $\Psi_{m n}$ and $\Psi_{p q}$ are barrier basin modes. Using the divergence theorem, no normal flow conditions, and in the case of barrier basin modes, the circulation con- 
straint about the barrier, $\oint \vec{u} \cdot d \vec{s}=0$, we obtain

$$
\left(\omega_{m n}-\omega_{p q}\right) \int_{D} \vec{\nabla} \Phi_{m n} \cdot \vec{\nabla} \Phi_{p q}^{*} d x d y=0
$$

which implies that if $\omega_{m n} \neq \omega_{p q}$ then the modes $\Psi_{m n}$ and $\Psi_{p q}$ are spatially orthogonal in the inner product defined by

$$
<\nabla f: \nabla g>=\int_{D} \vec{\nabla} f \cdot \vec{\nabla} g^{*} d x d y
$$

Using the divergence theorem and the appropriate boundary conditions we can show that the inner product (2.13) is equivalent to the inner product (2.15)

$$
\int_{D} \Phi_{m n} \nabla^{2} \Phi_{p q}^{*} d x d y
$$

which we define by

$$
<f: \nabla^{2} g>=\int_{D} f \cdot \nabla^{2} g^{*} d x d y
$$

\subsection{Projection of Solution Onto the Basin Modes}

Since we have a complete orthogonal set of eigenmodes and we know the inner product for which the spatial components of these modes are orthogonal, we can express any solution, $\Psi_{T O T A L}(x, y, t)$, to our forced basin problem as a series of normal modes.

For example, using the form of the inner product (2.15), we obtain

$$
\Psi_{\text {TOTAL }}(x, y, t)=\sum_{m=0}^{\infty} \sum_{n=0}^{\infty}\left(A_{m n} \Phi_{m n}+A_{m n}^{*} \Phi_{m n}^{*}\right)
$$

Equivalently,

$$
\Psi_{\text {TOTAL }}(x, y, t)=\sum_{m=0}^{\infty} \sum_{n=0}^{\infty} \Re\left(A_{m n} \Phi_{m n}\right)
$$

If $\Psi_{\text {TOT AL }}(x, y, t)$ were a solution for the barrier-free basin the $\Phi_{m n}$ would represent the spatial components of the barrier-free basin modes. Likewise, if $\Psi_{T O T A L}(x, y, t)$ were a solution for the barrier basin the $\Phi_{m n}$ would represent the spatial components 
of the barrier basin modes.

The series coefficients, $A_{m n}$, are time dependent and equal to the projection of the solution onto the spatial mode $\Phi_{m n}$ normalized by the norm of $\Phi_{m n}$. That is

$$
A_{m n}=\frac{\left\langle\Psi_{T O T A L}(x, y, t): \nabla^{2} \Phi_{m n}>\right.}{\left.<\Phi_{m n}: \nabla^{2} \Phi_{m n}\right\rangle}
$$




\section{Chapter 3}

\section{Linear Barrier Basin Problem}

Our first results concern the analytical solution to the linear, steady periodic forced barrier basin problem on the $\beta$-plane. The forcing is contained in the eastern subbasin along a meridional line at the coordinate $x_{F}$. A small amount of bottom friction is present. The solution is examined as the meridional structure of the forcing and forcing frequency are varied and as the bottom friction is increased. This work has been published in Atherton [1].

\subsection{Analytical Solution}

The linear, quasi-geostrophic potential vorticity equation on the $\beta$-plane for a barotropic fluid with forcing and bottom friction is, see Pedlosky [5],

$$
\nabla^{2} \psi_{t}(x, y, t)+\beta \psi_{x}(x, y, t)=\frac{\vec{\nabla} \times \vec{\tau}(x, y)}{\rho H} e^{i \omega_{F} t}-r \nabla^{2} \psi(x, y, t)
$$

There is no vortex stretching term since the Rossby radius of deformation is assumed to be large in comparison to the horizontal length scale of the flow, assumed to be the length scale of the basin, $L$. This is equivalent to a rigid lid boundary condition.

The stream function is related to the pressure, $P$, by $\psi=\frac{1}{f_{o} \rho_{o}} P$, where $f_{o}$ is the reference value of the Coriolis parameter and $\rho_{o}$ is the constant density. The Coriolis parameter is given by $f=f_{o}+\beta y$ in the $\beta$-plane approximation. 
The forcing is periodic in time with a frequency $\omega_{F}$. For our present analysis we will let $F(x, y, t)=\frac{\vec{\nabla} \times \vec{\tau}(x, y, t)}{\rho H} e^{i \omega_{F} t}$. The variables are nondimensionalized as follows

$$
\begin{aligned}
t & =(\beta L)^{-1} t^{\prime} \\
(x, y) & =L\left(x^{\prime}, y^{\prime}\right) \\
F(x, y, t) & =F_{o} T\left(x^{\prime}, y^{\prime}, t^{\prime}\right) \\
\psi(x, y, t) & =\frac{F_{o} L \Psi\left(x^{\prime}, y^{\prime}, t^{\prime}\right)}{\beta}
\end{aligned}
$$

and the nondimensionalized form of $(3.1)$ is

$$
\nabla^{2} \Psi_{t}\left(x^{\prime}, y^{\prime}, t^{\prime}\right)+\Psi_{x}\left(x^{\prime}, y^{\prime}, t^{\prime}\right)=T\left(x^{\prime}, y^{\prime}, t^{\prime}\right)-\gamma \nabla^{2} \Psi\left(x^{\prime}, y^{\prime}, t^{\prime}\right)
$$

where $\gamma=\frac{r}{B L}$ is the dimensionless friction parameter. Precisely, $\gamma$ is equal to the Stommel layer $\frac{r}{\beta}$ divided by the length scale of the basin. The magnitude of the forcing is given by $F_{o}$. All of the dimensionless variables $\left(x^{\prime}, y^{\prime}, t^{\prime}\right)$ are of order one. For clarity, from this point on the prime superscripts will be omitted. As in chapter $2, \Psi$ represents the nondimensional stream function and $\psi$ represents the dimensional stream function.

Since we are solving for the solution after all transients have decayed it will oscillate at the forcing frequency. Hence, we make the following substitutions

$$
\begin{aligned}
\Psi(x, y, t) & =e^{-i\left(\omega_{F_{n . d .}}{ }^{\left.t+k\left(x-x_{I}\right)\right)} \phi(x, y)\right.} \\
T(x, y, t) & =e^{-i \omega_{F_{n . d .}}{ }^{t} T(x, y)}
\end{aligned}
$$

where $k=\frac{1}{2\left(\omega_{F_{n . d .}}+i \gamma\right)}$ and $x_{I}$ is the zonal position of the thin meridional barrier. Since the $\mathrm{QG}$ equation has been nondimensionalized, we have denoted the nondimensional forcing frequency as $\omega_{F_{n . d .}}=\frac{\omega_{F}}{\beta L}$.

The substitutions (3.4) and (3.5) result in the inhomogeneous Helmholtz equation 
(3.6) for the envelope function $\phi(x, y)$

$$
\nabla^{2} \phi(x, y)+k^{2} \phi(x, y)=\frac{i}{\Omega} e^{i k\left(x-x_{I}\right)} T(x, y)
$$

where $\Omega=\omega_{F_{n . d .}}+i \gamma$.

The forcing is assumed to be a delta function in the zonal direction at $x=x_{F}$. By insisting that the forcing be limited to $x=x_{F}$, we isolate it to the eastern subbasin, thereby allowing us to concentrate on how the Rossby wave propagates past the barrier into the western sub-basin. The flexibility in choosing $T_{n}$, see equation (3.7) below, allows us to excite modes with various meridional structures. The forcing, $T(x, y)$, and the complete structure of the envelope, $\phi(x, y)$, are represented by a Fourier sine series in $y$ as follows

$$
\begin{aligned}
\phi(x, y) & =\sum_{n=1}^{\infty} \phi_{n}(x) \sin (n \pi y) \\
T(x, y) & =\delta\left(x-x_{F}\right) \sum_{n=1}^{\infty} T_{n} \sin (n \pi y)
\end{aligned}
$$

The Fourier coefficients, $\phi_{n}(x)$, in equation (3.7) are functions of $x$ to be determined. The boundary condition of no normal flow at $y=0$ and $y=1$ is now satisfied since $\phi(x, y)$ has been expressed as a Fourier sine series, and hence vanishes at $y=0$ and $y=1$.

The above substitutions for $\phi(x, y)$ and $T(x, y)$ lead to the following second order ordinary differential equation in $x$ for each Fourier coefficient $\phi_{n}(x)$

$$
\frac{d^{2} \phi_{n}(x)}{d x^{2}}-\alpha_{n}^{2} \phi_{n}(x)=\frac{i}{\Omega} e^{i k\left(x-x_{I}\right)} T_{n} \delta\left(x-x_{F}\right)
$$

where $\alpha_{n}^{2}=n^{2} \pi^{2}-k^{2}$. The solution to this equation in the basin interior involves solving the second order homogeneous equation

$$
\frac{d^{2} \phi_{n}(x)}{d x^{2}}-\alpha_{n}^{2} \phi_{n}(x)=0
$$


in the three regions

$$
\begin{aligned}
0 & <x<x_{I} \\
x_{I} & <x<x_{F} \\
x_{F} & <x<1
\end{aligned}
$$

leading to a total of six unknown constants. Therefore, six boundary conditions are required to determine these constants. The condition of no normal flow at $x=0$ and $x=1$ requires the stream function to be zero at the basin walls. Recall that the no normal flow boundary conditions at $y=0$ and $y=1$ have already been satisfied by the sine series (3.7). Another two boundary conditions arise from matching the solution at the zonal position of the delta function of the forcing $x=x_{F}$. The stream function is continuous at this point and so provides the first of these conditions; integrating (3.9) from $x_{F}-\epsilon$ to $x_{F}+\epsilon$ and taking the limit as $\epsilon$ goes to zero yields the second matching condition for the first derivative of $\phi_{n}(x)$. The last two unknown coefficients are determined by assuming that the total stream function has the form

$$
\Psi\left(x_{I}, y, t\right)=e^{-i \omega_{F_{n . d .}}{ }^{t}}\left\{\begin{array}{crrr}
\frac{y}{d} \phi_{I} & \text { for } & 0<y<d \\
\phi_{I} & \text { for } & d<y<1-d \\
\frac{1-y}{d} \phi_{I} & \text { for } & 1-d<y<1
\end{array}\right.
$$

at $x=x_{I}$, where $\phi_{I}$ is the barrier constant such that the full stream function on the barrier is $\Psi\left(x_{I}, t\right)=e^{-i \omega_{F_{n . d .}}{ }^{t}} \phi_{I}$. A no normal flow condition at the barrier dictates that $\phi_{I}$ must be independent of $y$. It should be noted that $\phi_{I}$ is a function of the meridional structure of the forcing, the forcing frequency, and the friction parameter and must be determined as part of the solution. The form (3.11) holds for $d<<1$ and assumes the flow is zonal, independent of $y$ and time periodic in the region of the narrow gaps. See Pedlosky and Spall [4] for a comparison of this linear assumption to numerical results, without the presence of friction. The linear assumption is valid as long as the Stommel boundary layer is small compared to the gap width.

The assumed form for the stream function at $x=x_{I}$, see (3.11), is expanded in a Fourier sine series, and its Fourier coefficient functions, $\phi_{n}\left(x_{I}\right)$, are matched to the solutions of (3.10) in the regions $0<x<x_{I}$ and $x_{I}<x<x_{F}$ on the line $x=x_{I}$. 
At this point the Fourier coefficients, $\phi_{n}(x)$, are determined in terms of the forcing and $\phi_{I}$. The solutions for the Fourier coefficients of the envelope function are In the region $0<x<x_{I}$

$$
\phi_{n}(x)=E_{n} \sinh \alpha_{n}(x)
$$

In the region $x_{I}<x<x_{F}$

$$
\phi_{n}(x)=C_{n} \sinh \alpha_{n}\left(x-x_{I}\right)+D_{n} \cosh \alpha_{n}\left(x-x_{I}\right)
$$

In the region $x_{F}<x<1$

$$
\phi_{n}(x)=A_{n} \sinh \alpha_{n}(x-1)
$$

with

$$
\begin{gathered}
D_{n}=\frac{4 \phi_{I} \sin (n \pi d) M(n)}{n^{2} \pi^{2} d} \\
M(n)= \begin{cases}0 & \text { if } \mathrm{n}=\text { even } \\
1 & \text { if } \mathrm{n}=\text { odd }\end{cases} \\
C_{n}=\frac{1}{\alpha_{n} \sinh \alpha_{n}\left(1-x_{I}\right)}\left(\tilde{T}_{n} \sinh \alpha_{n}\left(x_{F}-1\right)-D_{n} \alpha_{n} \cosh \alpha_{n}\left(1-x_{I}\right)\right) \\
A_{n}=\frac{1}{\sinh \alpha_{n}\left(x_{F}-1\right)}\left(C_{n} \sinh \alpha_{n}\left(x_{F}-x_{I}\right)+D_{n} \cosh \alpha_{n}\left(x_{F}-x_{I}\right)\right) \\
E_{n}=\frac{D_{n}}{\sinh \alpha_{n} x_{I}}
\end{gathered}
$$

In equation (3.17), $\tilde{T}_{n}=\frac{i}{\Omega} e^{i\left(x_{F}-x_{I}\right)} T_{n}$. Finally, to determine the value of $\phi_{I}$, the circulation condition is found by integrating the tangential component of the horizontal momentum equation

$$
\frac{\partial \vec{u}}{\partial t}+(\zeta+f) \hat{k} \times \vec{u}=-\nabla\left(\frac{p}{\rho}+\frac{|u|^{2}}{2}\right)-r \vec{u}+\vec{F}
$$

about the barrier. Here we assume there is no forcing tangent to the barrier.

The integral of the second term on the left side is equal to zero due to the boundary 
condition of no normal flow. Note that, although we are solving a linear problem, the nonlinear part of the second term will also integrate to zero, and hence the circulation condition applies to nonlinear problems as well. The integral of the first term on the right side is also zero since it is the integral of a perfect differential. The two remaining terms lead to the ordinary differential equation (3.21) for the circulation integral, which is Kelvin's theorem applied on a contour encircling the barrier.

$$
\left(\frac{\partial}{\partial t}+r\right) \oint \vec{u} \cdot d \vec{s}=0
$$

The transient solutions decay exponentially in time leaving a steady periodic solution. Therefore, after the transients have decayed away, the circulation condition is

$$
\oint \vec{u} \cdot d \vec{s}=0
$$

Since the geometry of the barrier is a thin meridional line, the above integral becomes

$$
\int_{d}^{1-d} v_{+}\left(x_{I}\right) d y=\int_{d}^{1-d} v_{-}\left(x_{I}\right) d y
$$

where $v_{+}\left(x_{I}\right)=\left.\sum_{n=1}^{\infty} \frac{\partial \Psi_{+}}{\partial x}\right|_{x=x_{I}} \sin (n \pi y)$ and $v_{-}\left(x_{I}\right)=\left.\sum_{n=1}^{\infty} \frac{\partial \Psi}{\partial x}\right|_{x=x_{I}} \sin (n \pi y)$. The positive subscript refers to the solution just east of the barrier; likewise, the negative subscript refers to the solution just west of the barrier. After making the substitutions for the meridional velocities, a solution for the time independent part of the stream function on the barrier is obtained.

$$
\phi_{I}=\frac{\sum_{n=0 \mathrm{dd}}^{\infty} \frac{2 \tilde{T}_{n} \sinh \alpha_{n}\left(x_{F}-1\right) \cos (n \pi d)}{\pi n \sinh \alpha_{n}\left(1-x_{I}\right)}}{\sum_{n=\mathrm{odd}}^{\infty} \frac{\alpha_{n} \sin (n \pi d) \cos (n \pi d) \sinh \left(\alpha_{n}\right)}{n^{3} \pi^{3} d \sinh \alpha_{n}\left(1-x_{I}\right) \sinh \alpha_{n}\left(x_{I}\right)}}
$$

For the even components the equation is trivially satisfied and provides no information about $\phi_{I}$. Considering the solution in the western basin (3.12), we see that the Fourier coefficient functions are zero when $n$ is even, implying that there is no transport between the basin and the barrier for these modes. No transport 
between the barrier tip and the basin wall is equivalent to $\phi_{I}=0$. The conclusion is that even components, corresponding to motions which are antisymmetric about the mid-latitude $y=0.5$, make no contribution to the value $\phi_{I}$.

If the odd Fourier components of the forcing, $T_{n}$, are zero, it follows that $\phi_{I}=0$. Therefore, a forcing which is antisymmetric about $y=0.5$ will yield no flow through the gaps. From (3.12), it then follows that there would be no response in the western basin.

The full basin, eastern sub-basin, and western sub-basin variance of the response is calculated by integrating the absolute value of the square of the stream function over the area of the full basin, eastern sub-basin, and western sub-basin, respectively

$$
\text { variance }=\int_{D} \phi \phi^{*} d x d y
$$

The final expression for the variance along with an expression for the integral of the meridional velocity $v_{+}$on the eastern side of the barrier alone,

$$
\mathrm{EC}=\int_{d}^{1-d} v_{+}\left(x_{I}\right) d y
$$

is shown in the appendix $\mathrm{C}$. The complete solution for the stream function is given by

$$
\Psi(x, y, t)=e^{-i\left(\omega_{F_{n . d .}} t+k\left(x-x_{I}\right)\right)} \sum_{n=1}^{\infty} \phi_{n} \sin (n \pi y)
$$

\subsection{Results}

When the forcing frequency approaches a natural frequency of the system we have resonance, where the solution is dominated by a single mode. Since there is a small amount of bottom friction present we will obtain a finite resonant response. For the results of sections $3.2 .1,3.2 .2$, and 3.2 .3 the bottom friction is $\gamma=10^{-8}$. This small value was chosen so that we could examine well separated resonant solutions corresponding to forcings at the natural frequencies. As mentioned in section 3.1, the forcing structure is a delta structure in the zonal direction and by choosing the 
forcing coefficients, $T_{n}$, in equation (3.8) we determine the meridional structure of the forcing. In this chapter the meridional structure of the forcing was chosen to be expressed in terms of approximate delta functions. This was accomplished by letting $T_{n}=2 \sin \left(n \pi y_{0}\right) e^{-(1 / 10) n^{2}}$, where $y_{0}$ is the center of the approximate delta function. The factor $e^{-(1 / 10) n^{2}}$ makes the function an approximate, smoothed delta function by eliminating the higher terms in the summation of the Fourier series.

\subsubsection{Symmetric Forcing}

This forcing is symmetric about the mid-latitude of the basin. It is represented by a delta function at $x_{F}=0.07$ and an approximate delta function at $y_{0}=0.5$. Figure B-2 (a), (b) and (c) show the variance of the full basin, eastern sub-basin and the western sub-basin, respectively. The absolute value of the barrier constant, $\left|\phi_{I}\right|$, which indicates the magnitude of the flux through the gaps, is also plotted in these Figures by a dotted line. The peaks in the variance occur where there is a resonant solution.

Figure B-3 (a), (b), (c), (d), (e), (f), and (g) are the contour plots of the absolute value of envelope function, $|\phi(x, y)|$, corresponding to the first seven resonant solutions as the frequency is decreased. Note that, since the forcing was symmetric, only the full basin modes presented in section 2.2 are excited and hence these resonant solutions are dominated by a single full basin mode.

\section{A Subset of Symmetric Sub-Basin Modes}

Consider the solutions forced at the frequencies $\omega_{F_{n . d .}}=0.0479$ and $\omega_{F_{n . d .}}=0.0356$, see Figures B-3 (d) and (g). As we have explained, these resonant solutions are dominated by a single symmetric full basin mode described in section 2.2. In fact, since the forcing is symmetric about the mid-latitude for this linear problem, all the modes contributing to the response are full basin modes. Why then do the two full basin modes dominating the solutions in Figures B-3 (d) and (g) have such a strong resemblance to sub-basin modes in both frequency and spatial structure? At a glance, the contour plots in Figures B-3 (d) and (g) indicate that the solution at $\omega_{F_{n . d}}=0.0479$ might be an eastern sub-basin mode and the solution at $\omega_{F_{n . d .}}=0.0356$ might be a western sub-basin mode. Both these modes have a $(1,3)$ structure in their 
respective sub-basins. Their resemblançe to sub-basin modes manifests itself again in their resonant frequencies, $\omega_{F_{n . d .}}=0.0479$ and $\omega_{F_{n . d .}}=0.0356$, which are equal to the resonant frequencies of the mode $(1,3)$ in barrier-free basins the size of the eastern and western sub-basins respectively.

Clearly, the dominant barrier modes at the forcing frequencies $\omega_{F_{n . d .}}=0.0479$ and $\omega_{F_{n . d .}}=0.0356$ have properties of sub-basin barrier modes. Yet, as explained by the theory developed in section 2.2 , we know that barrier basin modes which are purely symmetric about the mid-latitude can not possibly be sub-basin modes which are antisymmetric about the mid-latitude.

Another indication that the barrier modes with natural frequencies 0.0479 and 0.0356 are not sub-basin modes is that in Figures B-3 (d) and (g) we see a small circulation in the western and eastern sub-basin, respectively. It is necessary to have a small circulation in the adjoining basin to satisfy the circulation condition about the barrier. Since each of these modes consists of three cells stacked in the meridional direction along a side of the barrier, with the circulation in the middle cell opposite to the circulation in the end cells, it is clear that there is a weak net circulation along the barrier which must be compensated for in the adjoining basin. The net circulation along the east side of the barrier is calculated as $E C=889.1022$ for $\omega_{F_{n . d .}}=0.0479$ and as $E C=-81.4408$ for $\omega_{F_{n . d .}}=0.0356$, see Figure B-3 (d) and $(\mathrm{g})$.

In the case of the sub-basin modes which are purely antisymmetric about the mid-latitude, a total cancellation of the flow can occur in the sub-basin and there is no need for any flow in the adjoining basin to satisfy the circulation condition. We will show in section 3.3 that it is impossible to excite a pure west sub-basin mode with a forcing contained in the eastern sub-basin. Hence, this is another indication that the resonant mode at $\omega_{F_{n . d .}}=0.0356$ is indeed a full basin mode.

Since the responses for the solutions forced at $\omega_{F_{n . d .}}=0.0479$ and $\omega_{F_{n . d .}}=0.0356$ are contained primarily in the eastern sub-basin or the western sub-basin, respectively, the value of the barrier constant does not peak with the variance at these frequencies; the reason being that there is not very much transport through the gaps, $d$, associated with these full basin barrier modes, see Figures B-5 (a), (b), and (c). 
We conclude that these modes at $\omega_{F_{n . d .}}=0.0479$ and $\omega_{F_{n . d .}}=0.0356$ are not in the set of antisymmetric sub-basin modes in section 2.2 , but they do form a subset of sub-basin "like" modes in the set of full basin modes.

\subsubsection{Antisymmetric Forcing}

The antisymmetric forcing was constructed with a zonal delta function at $x_{F}=0.07$ and an approximate delta function at $y_{0}=0.75$ minus an approximate delta function at $y_{0}=0.25$. The western sub-basin variance and the barrier constant, $\phi_{I}$, were zero for all forcing frequencies since there is no flow through the gaps. Figures B-4 (a) and (b) show the full basin and eastern sub-basin variance, respectively. Figures B-4 (c) and (d) show the contours of the envelope function for the first two resonant frequencies as the forcing frequency decreases. Note that the resonant solutions are antisymmetric and contained only in the eastern sub-basin. Since the forcing is antisymmetric the solution is composed only of sub-basin modes. In section 3.3 we will show why western sub-basin modes can not be excited by a forcing contained in the eastern basin even though its structure is antisymmetric about the mid-latitude.

\subsubsection{Asymmetric Forcing}

The asymmetric forcing was created by an approximate delta function at $x_{F}=0.7$ and a delta function at $y=0.75$. The full basin, eastern sub-basin and western sub-basin variance along with the absolute value of the barrier constant, $\left|\phi_{I}\right|$, are presented in Figures B-5 (a), (b), and (c). When there is a resonant mode which involves a large flux through the gap the absolute value of the barrier constant peaks with the variance. When there is a resonant mode which does not involve much flow through the gap the absolute value of the barrier constant remains small while the variance is large. The contour plots of the absolute value of the envelope function corresponding to the resonant solutions are presented in Figures B-6 (a), (b), (c), (d), (e), (f), (g), (h), and (i). Since we have an asymmetric forcing, both symmetric full basin modes and antisymmetric sub-basin modes will contribute to our solution. 
Hence, with this forcing the resonant solution could be dominated by either a full basin or sub-basin mode. From the nine resonant solutions we have considered in Figure B-6 seven of them $((\mathrm{a}),(\mathrm{b}),(\mathrm{d}),(\mathrm{e}),(\mathrm{g}),(\mathrm{h})$, and (i)) are dominated by the same resonant full basin modes of the symmetric forcing in section 3.2 .1 ; two of them, (e) and (f), are dominated by the same resonant sub-basin modes in section 3.2.2. Comparing the values of the circulation along the eastern side of the barrier, EC, of

the asymmetric forced full basin modes, Figures B-6 (a), (b), (d), (e), (f), (g), (h), and (i) to the values of EC for the symmetrically forced modes Figures B-3, we see that the presence of antisymmetric modes in the asymmetric solutions has caused the circulation along the east side to decrease.

Almost all of these nine resonant frequencies are nearly equal to a barrier-free basin resonant frequency. In the case where the response is strongest in a sub-basin, the resonant frequency is equal to an eigenfrequency for a barrier-free basin the size of the sub-basin. Surprisingly, most of the barrier basin resonant solutions also resemble the barrier-free basin modes in terms of their structures. Table A.1 lists a comparison of the barrier modes and the corresponding barrier-free basin modes which they resemble. Note that we have two full barrier basin modes, at frequencies of $\omega_{F_{n . d}}=0.039$ and $\omega_{F_{n . d .}}=0.0381$, which do not correspond to any of the natural frequencies of the corresponding barrier-free basin. A closer look at their contour plots, see Figures B-6 (g) and (h), demonstrates that they also have no structural resemblance to any of the barrier-free modes.

\subsubsection{Bottom Friction}

When the bottom friction is increased the resonant solutions diminish in the magnitude of their response. The peaks in the variance associated with the resonant solutions broaden in frequency and eventually the peaks merge together and then disappear. These effects are demonstrated in Figures B-7 (a), (b), (c), (d), (e), and (f), which plot the full basin variance as the bottom friction parameter $\gamma$ is increased from $\gamma=0.0001$, in (a), up to $\gamma=0.0167$, in (f).

As one would probably expect, the effect of increasing the friction at a resonant 
forcing frequency is to diminish the response of the basin. This can be observed for the first full basin mode in Figures B-7 whose resonant peak diminishes in size each time the friction is increased. However, increasing the bottom friction can actually increase the response for certain non-resonant forcing frequencies. The nondimensional forcing frequency $\omega_{F_{n . d .}}=0.08$ with a symmetric forcing about the mid-latitude was chosen as an example to demonstrate this. Figures B-8 (a), (b), and (c) plot the full basin variance, eastern sub-basin variance, and the western sub-basin variance vs the bottom friction parameter $\gamma$, respectively. It is obvious, see Figure B-8 (a), that as the friction increases and the resonant peaks merge the response of the basin at this off resonant frequency increases up until $\gamma=0.002$. When the bottom friction increases further the response diminishes again. The reason for this is that the frequency $\omega_{F_{n} . d .}=0.08$ lies between the two resonant solutions of the first two full basin modes, $\omega_{n . d}=0.01057$ and $\omega_{n . d}=0.0686$. As the friction is increased and the resonant peaks, $\omega_{n . d .}=0.01057$ and $\omega_{n . d .}=0.0686$, become shorter and wider, the variance in the valley of frequencies about $\omega_{F_{n . d}}=0.08$ between these two peaks actually increases. After a certain point, $\gamma=0.002$, further increases in friction cause the overall solution to decrease.

Another interesting result, demonstrated in Figures B-8 (b) and (c), is that the response in the eastern sub-basin and western sub-basin differ as the friction is increased. The eastern sub-basin response diminishes as the friction is increased. Meanwhile the western sub-basin response initially increases in magnitude until $\gamma=0.002$ and then decreases thereafter. The different behaviors for the eastern and western sub-basins indicate that qualitative features of the solution are changing as the bottom friction is increased. Figure B-9 (a), (b), (c), and (d) demonstrates that this is indeed the case. It appears that when the bottom friction is low the response is dominated by the second full basin mode, see Figure B-9 (a). As the bottom friction is increased the response is dominated more by the first full basin mode, see Figure B-9 (d). This demonstrates that as friction is increased, resulting in the first two resonant peaks merging together, the dominant full basin mode is the first full basin mode. 


\subsection{Analysis - Another Look at the QG Equation}

We have solved the linear steady periodically forced problem for a barrier basin and have examined the results for various meridional forcing structures, forcing frequencies and increasing bottom friction. In this section we will analyze the results in terms of the basin modes we found in section 2.2. In particular, we will illuminate why it is impossible to excite a western sub-basin mode from a forcing in the eastern subbasin, and why symmetric forcing only excites the symmetric full basin modes and antisymmetric forcing only excites antisymmetric sub-basin modes. In section 2.4 we saw that a solution can be expressed as a series of eigenmodes, see equation (2.17). Substituting this series into the nondimensional QG equation we obtain

$$
\begin{array}{r}
\frac{\partial}{\partial t} \nabla^{2}\left(\sum_{m=0}^{\infty} \sum_{n=0}^{\infty}\left(A_{m n} \Phi_{m n}+A_{m n}^{*} \Phi_{m n}^{*}\right)\right)+\frac{\partial}{\partial x}\left(\sum_{m=0}^{\infty} \sum_{n=0}^{\infty}\left(A_{m n} \Phi_{m n}+A_{m n}^{*} \Phi_{m n}^{*}\right)\right)= \\
\frac{\vec{\nabla} \times \vec{\tau}}{\rho H} \mathrm{e}^{i \omega_{F_{n . d .}} t}-\gamma \nabla^{2}\left(\sum_{m=0}^{\infty} \sum_{n=0}^{\infty}\left(A_{m n} \Phi_{m n}+A_{m n}^{*} \Phi_{m n}^{*}\right)\right)
\end{array}
$$

Using the fact that the $\Psi_{m n}$ 's solve (2.1) exactly we have

$$
\begin{aligned}
-i \omega_{m n} \nabla^{2} \Phi_{m n} & =\frac{\partial}{\partial x} \Phi_{m n} \\
i \omega_{m n} \nabla^{2} \Phi_{m n}^{*} & =\frac{\partial}{\partial x} \Phi_{m n}^{*}
\end{aligned}
$$

where $\omega_{m n}=\frac{1}{2 k_{m n}}=\frac{1}{2 \pi \sqrt{m^{2}+n^{2}}}$. Substituting (3.28) and (3.29) into (3.27) and rearranging we obtain

$$
\begin{aligned}
\sum_{m=0}^{\infty} \sum_{n=0}^{\infty}\left(\left(\frac{\partial A_{m n}}{\partial t}-\left(i \omega_{m n}-\gamma\right) A_{m n}\right) \nabla^{2} \Phi_{m n}\right. & + \\
\left.\left(\frac{\partial A_{m n}^{*}}{\partial t}-\left(-i \omega_{m n}-\gamma\right) A_{m n}^{*}\right) \nabla^{2} \Phi_{m n}^{*}\right) & =\frac{\vec{\nabla} \times \vec{\tau}}{\rho H} \mathrm{e}^{i \omega_{F_{n . d .}} t}
\end{aligned}
$$

Taking the projection of equation (3.30) onto a particular mode $\Phi_{p q}$, we obtain an ordinary differential equation for the time evolution of the amplitude of the $(p, q)$ th mode $A_{p q}$. Therefore, multiplying the summation (3.30) by $\Phi_{p q}^{*}$ and integrating over 
the area of the basin, we have

$$
\frac{\partial A_{p q}}{\partial t}-\left(i \omega_{p q}-\gamma\right) A_{p q}=\frac{<\frac{\vec{\nabla} \times \vec{r}}{\rho H}: \Phi_{p q}>}{\left\langle\nabla^{2} \Phi_{p q}: \Phi_{p q}>\right.} e^{i \omega_{F} F_{n . d .}} t
$$

Equation (3.31) demonstrates that only modes which project onto the forcing structure will be excited. This explains why the symmetric forcing could only excite symmetric full basin modes and why the antisymmetric forcing could only excite the antisymmetric sub-basin modes. Since the forcing is only in the eastern sub-basin, this feature also explains why no western sub-basin modes could be excited even with an antisymmetric forcing. Western sub-basin modes are zero in the eastern sub-basin and the forcing is zero in the western sub-basin, hence the forcing simply does not project onto any western sub-basin modes.

Solving equation (3.31) we get the solution for the time evolution of a particular mode $A_{p q}$.

$$
\begin{aligned}
A_{p q}(t)= & \left(\frac{<\frac{\vec{\nabla} \times \vec{\tau}}{\rho H}: \Phi_{p q}>}{<\nabla^{2} \Phi_{p q}: \Phi_{p q}>} \frac{1}{\left(i\left(\omega_{F_{n . d .}}-\omega_{p q}\right)+\gamma\right)}\right) \mathrm{e}^{i \omega_{F_{n . d .}} t} \\
& +\left(A_{p q}(0)-\frac{<\frac{\vec{\nabla} \times \vec{\tau}}{\rho H}: \Phi_{p q}>}{<\nabla^{2} \Phi_{p q}: \Phi_{p q}>} \frac{1}{\left(i\left(\omega_{F}-\omega_{p q}\right)+\gamma\right)}\right) \mathrm{e}^{i \omega_{p q} t-\gamma t}
\end{aligned}
$$

As we can see, there is a particular, steady periodic, solution which oscillates at the forcing frequency and becomes very large when the forcing frequency, $\omega_{F n . d .}$, is close to the natural frequency of the system, $\omega_{p q}$. It is this particular solution that we are studying.

There is also the homogeneous, transient, solution, oscillating at $\omega_{p q}$ which is present to satisfy the initial conditions $A_{p q}(0)$, and which decays as $\mathrm{e}^{-\gamma t}$. Note that all the modes decay at the same rate. If we had used a higher order friction, such as lateral friction, the modes in the transient would have decayed at different rates depending on their length scales.

Also note that before the transient solution decays it will interact with the particular solution to create a beat phenomena, during the spin up portion of the problem. 


\section{Chapter 4}

\section{Numerical Model}

Before presenting the nonlinear model results we will discuss the model used for the computations and the parameters which were chosen for the runs.

\subsection{MICOM Model}

The nonlinear solutions have been computed numerically using the MICOM primitive equation isopycnal model. The hydrostatic approximation is used in MICOM. Hence, it is described as a "stacked shallow water model"; each layer is stacked above another, obeying the shallow water equations. Our homogeneous problem is modeled by a single layer of the MICOM model. The momentum and continuity equations are as follows

$$
\begin{aligned}
\frac{D \vec{u}}{D t}+f \hat{k} \times \vec{u} & =-g \vec{\nabla} h+\frac{\vec{\tau}}{\rho H}+A_{H} \nabla^{2} \vec{u}-r \vec{u} \\
\frac{\partial h}{\partial t}+\vec{\nabla} \cdot(h \vec{u}) & =0
\end{aligned}
$$

Here the total height of the fluid is given by $h=H+\eta$, where $H$ is the average height of the fluid and $\eta$ is the free surface height of the fluid. Equations (4.1) and (4.2) are solved numerically on a $\beta$-plane. Since we have taken the length of the basin to be $2000 \mathrm{~km}$, we are "pushing" the $\beta$-plane approximation. The discretization is an Arakawa "C" grid. 
To ensure that the model was operational with the inclusion of the barrier, its results of a very weakly nonlinear solution were matched to the analytical solution in chapter 3 .

\subsection{QG Dynamics}

We wish to examine our nonlinear problem in the context of QG dynamics with a rigid lid approximation. Considering the following dimensional shallow water potential vorticity equation (4.3), we will demonstrate how QG dynamics are approximated by MICOM. Equation (4.3) is derived by taking the curl of the momentum equation (4.1) and using the continuity equation (4.2) with the substitution of $-\frac{1}{h} \frac{D h}{D t}$ for $\nabla \cdot \vec{u}$.

$$
\frac{D}{D t}\left(\frac{\zeta+f \hat{k}}{h}\right)=\frac{A_{H}}{h}\left(\nabla^{2} \zeta\right)-\frac{r}{h} \zeta+\frac{(\vec{\nabla} \times \vec{\tau}) \cdot \hat{k}}{\rho H h}
$$

The relative vorticity is the vertical component of the curl of the horizontal velocities, $\zeta=(\vec{\nabla} \times \vec{u}) \cdot \hat{k}$. As one would expect, the total time derivative of the potential vorticity depends on the lateral friction, bottom friction and wind forcing. Following the assumptions of QG dynamics, we can approximate the shallow water potential vorticity, $\frac{\zeta+f \hat{k}}{h}$, by the $\mathrm{QG}$ potential vorticity. Assuming a small Rossby number, we have the geostrophic approximation for the first order velocity $f \hat{k} \times \vec{u} \sim \frac{1}{\rho} \vec{\nabla} P$, see Pedlosky [5]. Recalling that the fluid is hydrostatic, $P \sim g \rho h$, we scale the surface height accordingly, $\eta \sim O\left(\frac{f U L}{g}\right)$.

Let the height of the fluid be $h=H\left(1+\frac{N \eta^{\prime}}{H}\right)$, where $N$ scales the surface displacement $\eta$. Then we nondimensionalize the potential vorticity

$$
\frac{\zeta+f \hat{k}}{h} \sim \frac{f}{H}\left(\frac{R_{o} \zeta^{\prime}+1}{1+\frac{N \eta^{\prime}}{H}}\right)
$$

(here $R_{o} \sim O\left(\frac{U}{f L}\right)$ is the Rossby number, and ' indicates that a variable is nondimensional). Since $N<<H$, in $\mathrm{QG}$ dynamics, we can Taylor expand $\left(\frac{1}{1+\frac{N \eta^{\prime}}{H}}\right)$. Ignoring 
terms of order squared, we obtain

$$
\left(\frac{\zeta+f \hat{k}}{h}\right) \sim\left(\frac{f}{H}\right)\left(1+R_{0} \zeta^{\prime}-\frac{N \eta^{\prime}}{H}+\cdots\right)
$$

We have kept the planetary vorticity term, the relative vorticity term, and the vortex stretching term. Expression (4.4) multiplied by $H$ is the form the potential vorticity takes in the QG equation. To carry out the Taylor expansion it was necessary that $\frac{N}{H}$ be small. Now $\frac{N}{H}$ is a nondimensional parameter which can also be expressed in terms of the Rossby number, $R_{o}$, and the Froude number, $F_{r}$, since

$$
\frac{N}{H} \sim O\left(\frac{f U L}{g H}\right) \sim O\left(\frac{U}{f L} \frac{f^{2} L^{2}}{g H}\right) \sim R_{o} F_{r}
$$

The Froude number is equal to the length scale over the Rossby radius of deformation, $R_{D} \sim \frac{\sqrt{g \vec{H}}}{f}$, all squared, $\left(\frac{L}{R_{D}}\right)^{2}$.

\section{Rigid Lid}

Substituting (4.5) into (4.4), we have the QG vorticity as

$$
\frac{f}{H}\left(1+R_{o} \zeta^{\prime}-R_{o} F_{r} \eta^{\prime}+\ldots\right)
$$

To assume a rigid lid we need the term $R_{0} F_{r} \eta^{\prime}$ to be small. Specifically, it should be much smaller than $R_{o} \zeta^{\prime}$ and, hence, we need a small Froude number. Thus, we require $L<<R_{D}$ in order to approximate a rigid lid. In our model runs we have $L=2000 \mathrm{~km}$ and $R_{D}=\frac{\sqrt{\left(9.8\left[\mathrm{~m} / \mathrm{s}^{2}\right]\right)(16000[\mathrm{~m}])}}{0.5 \times 10^{-4}[1 / \mathrm{s}]} \sim 8000 \mathrm{~km}$. The Froude number remains small as we vary the Rossby number of the flow. Note that the ratio $\frac{\vec{\nabla} \cdot \vec{u}}{\zeta}$ scales as $\frac{N}{H}$, since $\zeta$ scales as $\frac{U}{L}$ and $\vec{\nabla} \cdot \vec{u}$ scales as $\frac{N U}{H L}$ from the continuity equation (as long as the time scale is not smaller than the advective time scale, $\frac{U}{L}$, and the geostrophic and hydrostatic approximations hold). Hence $\frac{\vec{\nabla} \cdot \vec{u}}{\zeta}$ also scales as $R_{o} F_{r}$.

To check the rigid lid approximation we consider the terms $\eta_{t}$ and $H(\vec{\nabla} \cdot \vec{u})$ from the continuity equation, which scale as $R_{o} F_{r}$ and $R_{o}$, respectively. Hence, $\frac{\eta_{t}}{H(\vec{\nabla} \cdot \vec{u})}$ scales as $F_{r}$. A small Froude number indicates that the rigid lid approximation holds.

Note that, since we have approximated a rigid lid, there is minimal change in the 
potential energy of the motion. Hence, we estimate the total energy to be the kinetic energy and have used the mean integrated kinetic energy as a measure of the variance of the flow.

Also, since there is a rigid lid, the flow in this one-layer model should be nondivergent (this of course has been checked from the output data) and, therefore, there is a stream function describing the flow. We integrate the meridional velocity to obtain this stream function, which is contoured in subsequent chapters to present our model results. The stream function exists even in our turbulent regime due to the fact that the flow is always non-divergent.

\section{QG Regime}

As we increase the forcing strength, or as we approach resonance, do we remain in the QG regime? To answer this, it is necessary to consider the Rossby number of the flow. Typically a Rossby number of less then 0.2 is considered to be in the QG regime of the shallow water equations.

As we will see in section 4.4, our parameter $\left(\frac{\delta_{I}}{L}\right)_{W N}^{2}$ is an accurate estimate for the relevant Rossby number of flow when the solution is weakly nonlinear and far from resonance. Since we change $\left(\frac{\delta_{I}}{L}\right)_{W N}^{2}$ from 0.002 to 0.01 we can safely say that we are in the QG regime for these solutions. However, as the forcing increases and the scales of motion change, $\left(\frac{\delta_{I}}{L}\right)_{W N}^{2}$ will no longer be an accurate estimate of the Rossby number. The question is whether or not the Rossby number is still small enough to be in the QG regime. Of course, the Rossby number will vary throughout the basin and the only way to get an estimate of the Rossby number for these solutions is to estimate it directly from the model output.

Note that when the solution is in the QG regime we would normally expect the stream function to be $\frac{g \eta}{f_{0}}$. However, we have such a large $\beta$-plane that it is likely that the variation in $f$ in the shallow water equations can not be ignored. Most likely we have $u=-\frac{g \partial \eta}{f(y) \partial y}$ and $v=\frac{g \partial \eta}{f(y) \partial x}$ and, hence, the stream function is not directly proportional to the surface height $\eta$.

Unlike the $\mathrm{QG}$ equation, the shallow water equations on the $\beta$-plane are not invariant with respect to $y$. Thus, we might see some asymmetry in the solution 
about the mid-latitude which we would not expect to see in the QG model.

\subsection{Numerical Stability and Lateral Friction}

When conducting the nonlinear experiments it is necessary, to ensure numerical stability, to include the higher order lateral friction. Unlike bottom friction, which is scale independent, lateral friction dissipates the smaller scales at a much higher rate. The value we have chosen is $A=800\left[\mathrm{~m} / \mathrm{s}^{2}\right]$. The simple calculation below shows that for grid sized motions the dissipation by lateral friction is actually greater than the bottom friction dissipation. Balancing $\frac{\partial \nabla^{2} \Psi}{\partial t} \sim-r \nabla^{2} \Psi$ and $\frac{\partial \nabla^{2} \Psi}{\partial t} \sim-\frac{A}{L^{2}} \nabla^{2} \Psi$ we find that the bottom friction diminishes $\nabla^{2} \Psi$ as $e^{-r t}$ and, likewise, lateral friction as $e^{-\frac{A}{L_{G}^{2}} t}$. The length scale, $L_{G}$, used is twice the grid size of $20 \mathrm{~km}$. We chose $r=8 \times 10^{-8}[1 / s]$ and $\frac{A}{L_{G}^{2}}=5 \times 10^{-7}[1 / s]$. This implies a time scale of 145 days for the bottom friction and about 23 days for the lateral friction. On the other hand, when considering basin sized motions, the bottom friction still has a time scale of 145 days and the lateral friction has a time scale of 57870 days.

Since we are studying the nonlinear solution for the first few Rossby modes (which have basin-sized scales), the solutions we are considering are of large scale. The lateral friction is used for numerical stability and the bottom friction is used to dissipate the initial conditions, see equation (3.32), thereby leaving only the steady periodic forced solution. We chose a value of bottom friction which was low enough to keep the resonant peaks well separated. That allows us to clearly see what role the nonlinearity played in the reduction of the peak.

The value of the lateral friction is very small. In addition, it is difficult to obtain an analytical solution when lateral friction, which raises the order of the QG equation, is included, even in the linear case. The model was run using no normal flow $(\vec{u} \cdot \hat{n}=0)$ and slip $(\zeta=0)$ boundary conditions. The lateral friction was kept constant for numerical stability and the effects of changing the magnitude of the lateral friction or varying the boundary conditions were not studied. The slip conditions were chosen to minimize the shear and, thus, minimize the production of eddies at the boundary. 
Therefore, we could focus on the eddies produced by the nonlinearities at the tips of the barrier.

\section{Circulation Condition}

By integrating the momentum equation, in the presence of the lateral friction term, about the barrier, as was done in section 3.1, we obtain the circulation condition including the effects of lateral friction.

$$
\frac{\partial}{\partial t} \oint u \cdot d l=-r \oint u \cdot d l+A \oint \nabla \zeta \cdot \hat{n} d l
$$

If we have superslip boundary conditions $(\nabla \zeta \cdot \hat{n}=0)$, see Pedlosky [6], then the contribution of lateral friction is zero and we have the same boundary condition as before. Since we are not using superslip boundary conditions, it is clear that the no net circulation condition is affected by the lateral friction. However, since we have used such a small value of lateral friction for numerical reasons, we assumed the effect to be negligible. Since MICOM has a staggered grid, it is difficult to accurately estimate the circulation about the barrier or to check the effect of lateral friction on this circulation condition. If we had considered a wind forcing above the barrier this would have further affected the no net circulation condition.

\subsection{Scaling for the Numerical Runs}

Since it seems impossible to obtain a uniform scaling for a turbulent solution because of the presence of many diverse scales of motion, we base our scaling on the weakly nonlinear solution, for which a single scale dominates. To simplify this process we scale the $\mathrm{QG}$ equation and not the shallow water equations.

Therefore, we begin with the QG equation, expressed such that the planetary vorticity is separate from the relative vorticity.

$$
\nabla^{2} \psi_{t}+\beta \psi_{x}+J\left(\psi, \nabla^{2} \psi\right)=\frac{\vec{\nabla} \times \vec{\tau}}{\rho H} \mathrm{e}^{i \omega_{F} t}-r \nabla^{2} \psi+A \nabla^{4} \psi
$$

In the range of our forcing frequencies, $\omega_{F}$, we can nondimensionalize the variables 
as follows

$$
\begin{aligned}
t & =\left(\omega_{F}\right)^{-1} t^{\prime} \\
(x, y) & =L\left(x^{\prime}, y^{\prime}\right) \\
\frac{\vec{\nabla} \times \vec{\tau}}{\rho H} \mathrm{e}^{i \omega_{F} t} & =\frac{\tau}{L \rho H} F\left(x^{\prime}, y^{\prime}, t^{\prime}\right) \\
\psi(x, y, t) & =\psi \Psi\left(x^{\prime}, y^{\prime}, t^{\prime}\right)
\end{aligned}
$$

Here the prime, ${ }^{\prime}$, indicates that the variable is nondimensional. The lower case $\psi$ represents both the dimensional stream function and the scaling for the nondimensional stream function. Expressing equation (4.8) in terms of the nondimensional variables and multiplying by $\frac{L}{\beta \psi}$ so that the planetary vorticity term is of order one, we obtain, after dropping the primes,

$$
\omega_{F n . d .} \nabla^{2} \Psi_{t}+\Psi_{x}+\left(\frac{\delta_{I}}{L}\right)^{2} J\left(\Psi, \nabla^{2} \Psi\right)=\frac{\tau}{\rho H \beta \psi} F-\frac{\delta_{S}}{L} \nabla^{2} \Psi+\frac{\delta_{M}^{3}}{L^{3}} \nabla^{4} \Psi
$$

The nondimensional frequency $\omega_{F n . d .}$ is equal to $\frac{2 \pi}{T_{F} \beta L}$, and $T_{F}$ is the time scale or period of the motion, which we are assuming for the purpose of this scaling is the same as the time period of the forcing (of course as the solution becomes more and more nonlinear this won't be true). In addition, $\delta_{I}=\sqrt{\frac{\psi}{\beta L}}$ and $\delta_{S}=\frac{r}{\beta}$. Previously, see chapter $3, \frac{\delta_{S}}{L}=\gamma$. Since the Rossby modes naturally satisfy the boundary condition of no normal flow, in most of the solutions we are considering there will be no boundary layers. However, for the sake of clarity, I have used the traditional notation for these parameters; $\delta_{I}$ is the inertial boundary layer and $\delta_{S}$ is the Stommel boundary layer form the steady ocean circulation problem.

Each solution is characterized by the five following independent nondimensional parameters

$$
\begin{array}{rlrl}
\omega_{F n . d .} & =\frac{\omega_{F}}{\beta L} & & \text { (based on the forcing frequency) } \\
\frac{\Psi_{\text {Sverdrup }}}{\Psi}=\frac{\tau}{\rho H \beta \psi} & \text { (based on size of the forcing strength) }
\end{array}
$$




$$
\begin{aligned}
\left(\frac{\delta_{I}}{L}\right)^{2} & =\frac{\psi}{\beta L^{3}} & & \text { (based on size of the nonlinear terms) (4.10) } \\
\frac{\delta_{s}}{L} & =\frac{r}{\beta L} & & \text { (based on size of bottom friction term) } \\
\frac{\delta_{M}^{3}}{L^{3}} & =\frac{A}{\beta L^{3}} & & \text { (based on size of lateral friction term) }
\end{aligned}
$$

Applying the Buckingham $\Pi$ theorem with variables $\psi, \beta, L, \omega_{F}, \frac{\tau}{\rho H}, r$, and $A$ and with units $s$ and $m$ (i.e. time and length) is an alternative way of demonstrating that our problem is described by the nondimensional parameters (4.10) given above.

Although $\rho, H, \tau, \beta$ and $\omega_{F}$ are known, the values of the parameters $\omega_{F n . d .},\left(\frac{\delta_{I}}{L}\right)^{2}$, $\frac{\tau}{\rho H \beta \psi}, \frac{\delta_{S}}{L}$ and $\frac{\delta_{M}^{3}}{L^{3}}$ are unknown until we have $L$ and $\psi$. In a turbulent solution not only might we not be in the regime of $\mathrm{QG}$ dynamics, but it seems impossible to predict $\psi$ and $L$ a priori. Also, there is not a fixed value for $\psi$ and $L$ since the motions change throughout the basin in a turbulent solution. However, if the solution is only weakly nonlinear and hence resembles the linear solution then we can easily predict $\psi$ and $L$.

For the purpose of assigning parameter values, particularly the wind forcing, for the model runs we chose to predict $\psi$ and $L$ when the solution is weakly nonlinear and far from resonance. In this situation the planetary scaling balances the forcing term, $O(1) \sim O\left(\frac{\tau}{\rho H \beta \psi}\right)$, and solving for $\psi$ we have the Sverdrup scaling

$$
\psi \sim O\left(\frac{\tau}{\rho H \beta}\right)
$$

We can also take the length scale of the motion, $L$, to be the basin size if we are assuming a weakly nonlinear solution. This is because our forcing frequency range is from just above the frequency of the $(3,1)$ mode to just below the frequency of the $(1,1)$ mode. The modes $(3,1),(2,1)$, and $(1,1)$, which our forcing frequencies span, all have basin sized scales of motions. Hence, if we use the Sverdrup approximation for $\psi$, and assume the length scale, $L$, to be the basin size, we can estimate the size of the nonlinear terms a priori based upon the wind forcing. Taking $L_{B}$ to be the basin size, we have

$$
\left(\frac{\delta_{I}}{L}\right)^{2} \sim \frac{\psi}{L^{3} \beta} \sim O\left(\frac{\tau}{\rho H \beta^{2} L_{B}^{3}}\right)
$$


We see that $\left(\frac{\delta_{I}}{L}\right)^{2}$ indicates the size of the nonlinear terms relative to the planetary vorticity term, which is set to be order one in the nondimensional equation (4.9). The estimate, $\frac{\tau}{\rho H \beta^{2} L_{B}^{3}}$, of $\left(\frac{\delta_{I}}{L}\right)^{2}$ is only accurate when the Sverdrup scaling, $\frac{\tau}{\rho H \beta}$, for $\psi$ and the basin scaling, $L_{B}$, for $L$ are true. That is, our estimate (4.12) for $\left(\frac{\delta_{I}}{L}\right)^{2}$ is accurate only for the weakly nonlinear situations far from resonance. When the solution begins to become turbulent, or if we are close to resonance, our nondimensional parameter $\frac{\tau}{\rho H \beta^{2} L_{B}^{3}}$ is no longer a reasonable estimate for $\left(\frac{\delta_{I}}{L}\right)^{2}$. To clarify this situation we will denote $\frac{\tau}{\rho H \beta^{2} L_{B}^{3}}$ by $\left(\frac{\delta_{I}}{L}\right)_{W N}^{2}$. The subscript WN reminds us that $\frac{\tau}{\rho H \beta^{2} L_{B}^{3}}$ is only equal to $\left(\frac{\delta_{I}}{L}\right)^{2}$ in the weakly nonlinear solution.

The parameters for the numerical runs were chosen by setting $\psi$ equal to the Sverdrup scaling and $L$ equal to the basin scale. Therefore, we assign values to the wind forcing strength, bottom friction, and lateral friction, according to what values we desire for the nondimensional parameters $\left(\frac{\delta_{I}}{L}\right)_{W N}^{2}, \frac{\delta_{M}^{3}}{L^{3}}$, and $\frac{\delta_{S}}{L}$. We will see in chapter 5 that for all the higher non-resonant forcing frequencies in our runs, the solution is weakly nonlinear and, hence, $\left(\frac{\delta_{L}}{L}\right)_{W N}^{2}=\left(\frac{\delta_{I}}{L}\right)^{2}$, the actual size of the nonlinear terms in the nondimensional equation (4.9). 


\section{Chapter 5}

\section{Nonlinear Solution for the Barrier-Free Basin}

The weakly nonlinear solution for the barrier-free basin has already been studied in Pedlosky [2] and [3]. In this chapter we will review the previous weakly nonlinear theory and present our model runs, which increase in nonlinearity until the solution becomes turbulent. We will also examine the hysteresis which occurs when we vary the initial conditions of our runs.

\subsection{Previous Weakly Nonlinear Results for the Pe- riodically Forced Barrier Basin}

The papers Pedlosky [2] and [3] deal with the nonlinear periodically forced solution for a homogeneous square basin on the $\beta$-plane. The former paper, Pedlosky [2], considers the situation where the nonlinearity is dominated by bottom friction and forcing. The steady component of the nonlinear solution is considered in detail as the forcing frequency and bottom friction parameters are varied. The latter paper, Pedlosky [3], considers a more nonlinear solution near resonance where the nonlinear terms dominate the bottom friction and forcing terms. Particular attention was paid to the hysteresis which occurs at forcing frequencies near resonance. 


\subsubsection{Bottom Friction and Forcing Larger than the Nonlin- earity}

The work Pedlosky [2], uses the same scaling of the QG equation that we have used in our runs, see section 4.4, except that time is nondimensionalized by $\beta L$ and not $\frac{1}{\omega_{F}}$. Hence, the nondimensional form of the QG equation is

$$
\nabla^{2} \Psi_{t}+\Psi_{x}+R_{o} J\left(\Psi, \nabla^{2} \Psi\right)=T-\delta \nabla^{2} \Psi
$$

Where $R_{o}=\frac{\tau}{\rho \beta^{2} H L^{3}}$ (the parameter $R_{o}$ is equal to $\left(\frac{\delta_{I}}{L}\right)_{W N}^{2}$ in our notation) is the relevant Rossby number of the flow; also, $\delta=\frac{r}{\beta L}$ is the nondimensional friction parameter equal to the Stommel boundary layer over the length scale of the basin. The stream function $\Psi$ is expressed as $\Psi=\Psi_{o}+R_{o} \Psi_{1}+R_{o}^{2} \Psi_{2}+\ldots$ and substituted into the QG equation (5.1). The assumption is that $R_{o}$ is very small and smaller than $\delta$. The first order solution, $\Psi$, to the equation

$$
\nabla^{2} \Psi_{t}^{0}+\Psi_{x}^{0}+\delta \nabla^{2} \Psi^{0}=T
$$

is then found exactly by expressing the solution as a Fourier sine series in $y$ and using the Laplace transform to solve for the zonally dependent Fourier coefficients. The solution also confirms that the modes form a complete set. The steady solution for the following order problem

$$
\nabla^{2} \Psi_{t}^{1}+\Psi_{x}^{1}+\delta \nabla^{2} \Psi^{1}=-J\left(\Psi^{0}, \nabla^{2} \Psi^{0}\right)
$$

is found by approximating the first order solution for different regimes of the forcing frequency (i.e. (i) $\omega_{F_{n . d .}}=\omega_{m n}$, (ii) $\omega_{F_{n . d .}} \neq \omega_{m n}$, and (iii) $\omega_{F_{n . d .}}=O(\delta)$ ).

To summarize, it was found that a steady periodic forcing could produce a steady time independent solution due to the nonlinear interactions. In Pedlosky [2] the steady component of the first order solution $\Psi^{1}$ was found. It was also shown that the Rossby modes form a complete set. Steady and unsteady boundary layers were 
also observed. In case (ii) for $\omega_{F_{n, d .}}>(2 n \pi)^{-1}$, oscillating boundary layers occur in the first order solution at both the eastern and western walls. For this case steady boundary layers occur on both meridional walls in the next order solution. For case (iii) there is an oscillating boundary layer on the western wall as part of the first order solution, as well as a steady boundary layer on the western wall as part of the next order solution.

Our numerical solutions are in a more nonlinear regime than this paper considers.

\subsubsection{Nonlinearity Larger than the Bottom Friction and Forc- ing}

The latter paper, Pedlosky [3], is concerned with the solution when the forcing frequency is very close to an eigenfrequency of the system. In this case the Sverdrup scaling for $\psi$ is not used. Instead of the Sverdrup scaling, the nonlinear terms squared, $\left(\frac{\delta_{I}^{2}}{L^{2}}\right)^{2}=\frac{\psi}{\beta L^{3}}$ are balanced with the forcing term $\left(\frac{\tau}{\rho H \beta \psi}\right)$. Thus, $\psi=\left(\frac{\tau \beta L^{6}}{\rho H}\right)^{1 / 3}$ and the relevant Rossby number, denoted by $\epsilon$ is expressed as $\epsilon=\left(\frac{\tau}{\beta^{2} \rho H L^{3}}\right)$ in terms of the forcing. Note that $\epsilon=\left(\left(\frac{\delta_{I}}{L}\right)_{W N}^{2}\right)^{1 / 3}$, that is $\epsilon$ equals the cube root of our estimate of the nonlinear terms for the weakly nonlinear solution in section 4.4 and, hence, also equals the cube root of $R_{o}$, the relevant Rossby number from the previous paper Pedlosky [2]. Thus the nonlinear term is larger near resonance since $\epsilon^{(1 / 3)}>>\epsilon$ for $\epsilon<<1$. The bottom friction is set such that $\frac{r}{\beta L}=\epsilon^{2} \delta$. Therefore, we have the following nondimensional equation.

$$
\nabla^{2} \Psi_{t}+\Psi_{x}=\epsilon\left(-J\left(\Psi, \nabla^{2} \Psi\right)\right)+\epsilon^{2}\left(T-\delta \nabla^{2} \Psi\right)
$$

It is obvious from (5.4) that, since we are forcing at an eigenfrequency, the time tendency relative vorticity term and the planetary vorticity term (both of which are of order one) almost cancel each other; that is they cancel each other to order $\epsilon$, leaving the nonlinearity and friction to balance the forcing. The equation is solved using the method of multiple scales. An amplitude response curve is found where the 
resonant peaks tilt towards higher frequencies as the system becomes more nonlinear. The tilt is due to the nonlinear interaction of the zeroth order, $\Psi^{0}$, and first order, $\Psi^{1}$, solutions. The amplitude of the response is limited by friction to order $\epsilon^{2}$. The physical reason for the tilt of the amplitude curve is that, in the nonlinear problem, the natural frequencies of the system are amplitude dependent.

This latter paper is more relevant to our numerical runs, which begin in the weakly nonlinear regime of this paper and increase in nonlinearity. Figure B-10 uses the method of Pedlosky [3] to plot an amplitude response curve for the parameters of our first run, $\left(\frac{\delta_{l}}{L}\right)_{W N}^{2}=0.002$. A hysteresis is predicted. For certain forcing frequencies, depending on the initial conditions, the solution can be either one of two possible stable solutions. Of course, we do not observe the unstable solution associated with the dotted line in Figure B-10.

\subsection{Numerical Results}

The resonant mode $(2,1)$, which has a nondimensional natural frequency of 0.0712 , is used as a study case to see how resonance is affected by increasing nonlinearity. This mode was chosen since it contains basin-sized scales of motion, which makes it easy to scale in the weakly nonlinear case. Another added feature is its large meridional scale; we wish to avoid meridional length scales of motion the size of the gap width for the barrier basin we will study in chapter 6 . The dimensional period corresponding to the nondimensional resonant frequency of 0.0712 is 25.5 days, that is of the order of a month. Using the MICOM model described in section 4.1, we have carried out a series of runs of increasing nonlinearity over the nondimensional forcing frequency range 0.0575 to 0.095 which surrounds the resonant frequency 0.0712 . In this thesis, each set of runs consists of a series of runs in which all the parameters are fixed, except for the forcing frequency (which varies from $\omega_{F n . d .}=0.0575$ to 0.095 ). As described in section 4.4, the frequency is nondimensionalized by $\beta L$. We have six sets of runs increasing in nonlinearity. The nonlinear parameter, $\left(\frac{\delta_{I}}{L}\right)_{W N}^{2}$, took on the values $0.002,0.003,0.004,0.006,0.008$ and 0.01 , with corresponding forcing 
strengths $1024\left[\mathrm{~cm}^{2} / \mathrm{s}^{2}\right], 1536\left[\mathrm{~cm}^{2} / \mathrm{s}^{2}\right], 2048\left[\mathrm{~cm}^{2} / \mathrm{s}^{2}\right], 3072\left[\mathrm{~cm}^{2} / \mathrm{s}^{2}\right], 4096\left[\mathrm{~cm}^{2} / \mathrm{s}^{2}\right]$ and $5120\left[\mathrm{~cm}^{2} / \mathrm{s}^{2}\right]$, respectively. The bottom and lateral friction remained fixed with $\frac{\delta_{S}}{L_{B}}=$ $\frac{r}{\beta L_{B}}=2 \times 10^{-3}$, and $\frac{\delta_{M}^{3}}{L_{B}^{3}}=\frac{A}{\beta L_{B}^{3}}=5 \times 10^{-6}$. Here, the length scale $L$ was chosen as the basin scale $L_{B}$. This corresponds to $A=800\left[\mathrm{~m}^{2} / \mathrm{s}\right]$ and $r=8 \times 10^{-8}[1 / \mathrm{s}]$. All these runs were initialized with zero initial conditions.

The forcing was symmetric about the mid-latitude since the Ekman pumping has the meridional structure $\sin (\pi y)$. It extends over a meridional strip of $200 \mathrm{~km}$ width on the east side of the basin between $1600 \mathrm{~km}$ and $1800 \mathrm{~km}$ from the west side.

The height, $H$, of the fluid is chosen to be $16 \mathrm{~km}$, see section 4.2 for further details. Finally the Coriolis parameter, $f_{o}$, and the $\beta$ parameter are chosen to be $f_{o}=5 \times 10^{-5}[1 / \mathrm{s}]$ and $\beta=2 \times 10^{-13}[1 /(\mathrm{s} \mathrm{cm})]$. The basin is square with a length of $2000 \mathrm{~km}$. The runs lasted for 1000 model days and the results were stored for the final 300 days. The resolution used was $20 \mathrm{~km}$ by $20 \mathrm{~km}$.

In our discussion of the results we make reference to a "jump" from a weakly nonlinear solution to a turbulent solution. At this point, we will briefly clarify what is meant by nonlinear and turbulent. This clarification will become more apparent as the discussion proceeds.

\section{Weakly Nonlinear Solution}

Weakly nonlinear solutions result when the solution is dominated by the linear solution and nonlinearity is a first, second, or higher order effect. We have already discussed the weakly nonlinear solutions, Pedlosky [2] and Pedlosky [3]. In the model runs the weakly nonlinear solution is dominated by a single Rossby mode whose eigenfrequency is close to the forcing frequency of the basin. We will see that there are very small contributions by other Rossby modes associated with the appearance of integral multiples of the forcing frequency in the frequency spectrum. In our model results the weakly nonlinear solutions occur predominantly at higher frequencies than the resonant frequency and have a smaller amplitude response then the turbulent solutions. Note that the lower forcing strengths of $\left(\frac{\delta_{r}}{L}\right)_{W N}^{2}=0.002$ and 0.003 correspond to weakly nonlinear solutions for all forcing frequencies. These weakly nonlinear solutions are in the QG regime and will be analyzed using the Rossby modes developed 
in chapter 2 .

\section{Turbulent Solutions}

The turbulent solutions occur at forcing frequencies which are close to resonance or below for the higher forcing strengths. They have a whole range of motion scales varying throughout the basin. The amplitude of the response is larger than for the weakly nonlinear solution. Inertial boundary layers appear along the western, northern, and southern boundaries. Many frequencies of motion appear which are not integer multiples of the forcing frequency.

Unfortunately, we do not know the origin of these frequencies nor what is their relationship to the forcing frequency or the basin mode natural frequencies. For example, if this hysteresis was part of a parametric resonance one might expect the frequencies to be rational multiples of the forcing frequency. Alternatively, these frequencies might result from the excitation of the other natural modes, or perhaps from instabilities of the flow. The spectrum of natural frequencies is just too dense to give any indication of what might be occurring in this transition.

In this chapter we refer to the solution "jumping" to a turbulent state as soon as frequencies which are not integer multiples of the forcing frequency appear. This is also the point at which we have to be careful about leaving the QG regime.

\subsection{Description of Results}

Figure B-11 plots the mean basin integrated kinetic energy vs the forcing frequency for each set of runs. There are "jumps" in the solution occurring at the forcing frequencies $\omega_{F_{n . d .}}$ equal to $0.075,0.08,0.0825$ and 0.085 at the forcing strengths, $\left(\frac{\delta_{I}}{L}\right)_{W N}^{2}$, equal to $0.004,0.006,0.008$ and 0.01 , respectively. This occurs in those cases for which the solution loses its resonant peak and "jumps" from a weakly nonlinear solution, which is dominated by the linear solution, to a turbulent solution having many different scales of motion.

Figures B-12 (a), (b), (c), and (d) show the envelope contours calculated over one period of forcing just before the solution "jumps" to its high amplitude, turbulent 
state. Likewise, Figure B-13 (a), (b), (c), and (d) show the solution just after the transition. The envelope contours are the root mean square (RMS) of the numerical solution. If the linear analytical solution were expressed as $\Psi=\mathrm{e}^{i(\omega t+k x)} \phi$, where $\phi$ is the complex envelope function equal to $R \mathrm{e}^{i \theta}$, then the RMS of the solution is equivalent to $\frac{R}{\sqrt{2}}$.

Figures B-12 (a), (b), (c), and (d) represent the weakly nonlinear solution which resembles the linear solution. We see in Figure B-13 (a), (b), (c), and (d) that in the turbulent solution the length scale of the motion varies throughout the basin and, in general, is much smaller than in the weakly nonlinear solution, whose length scale is the basin size.

In the linear and weakly nonlinear solutions, for the mode $(2,1)$ which we are studying, we observed large, tall, oval cells propagating to the west and disappearing when they hit the western wall. With the introduction of the advection of relative vorticity, we instead see eddies appearing in the vicinity of the east side of the basin, heading in a westward direction (having to maneuver about each other as they do) and then piling up on the western wall. Even though the solution is nonlinear we still see a periodic, fairly regular motion where the dominant period is the forcing period. This will be confirmed in section 5.4 by Fourier analysis.

We now discuss the turbulent solutions which we observe after the transition. As an example we use the solution forced at the frequency $\omega_{F_{n . d .}}=0.0725$ and strength $\left(\frac{\delta_{I}}{L}\right)_{W N}^{2}=0.004$. We will then consider how the solution further changes when the forcing strength is increased to $\left(\frac{\delta_{I}}{L}\right)_{W N}^{2}=0.01$.

Turbulent Solution $\omega_{F_{n} . d .}=0.0725$ and $\left(\frac{\delta_{I}}{L}\right)_{W N}^{2}=0.004$

Figure B-14 shows a sequence of snapshots for the case $\left(\frac{\delta_{I}}{L}\right)_{W N}^{2}=0.004$ and $\omega_{F_{n . d .}}=$ 0.0725 over one period of motion. The period corresponding to the nondimensional frequency 0.0725 is 25.08 days. The 12 contour plots in Figure B-14 are plotted at two day intervals and, thus, we are missing the last few days of the period. Had we plotted the final two day interval it would resemble the contours in (a). The dotted contours represent negative stream lines and the solid contours represent positive stream lines. Eddies corresponding to positive stream lines rotate clockwise (i.e. are anticyclonic) 
and correspond to negative vorticity whilst eddies corresponding to negative stream lines rotate counterclockwise (i.e. are cyclonic) and correspond to positive vorticity. Starting with Figure B-14 (a), we see that there are two cyclonic eddies against the western wall, one at the mid-latitude and the other in the south-west corner of the basin. Using the method of images, we expect that the eddy at the mid-latitude of the western wall has a tendency to move south along the wall. However, it appears to be pinned in place by two anticyclonic eddies to either side of it. It remains there until it finally disappears, see Figure B-14 (e). Meanwhile, the eddy in the south-west corner weakens but is still able to remain there until it is fed more positive vorticity, see Figure B-14 (h). Also, note that in Figure B-14 (h) we now have two anticyclonic eddies on the western wall, one at mid-latitude and one in the north-west corner of the basin. The same cycle we have just described for the two cyclonic eddies in Figure B-14 (a) repeats itself for these two eddies. The final result is two almost permanent eddies in the south-west and north-west corners of the basin.

In summary, we have seen a complicated periodic motion with the period of the forcing frequency. Although we know that there are many motions corresponding to different frequencies, see section 5.4, it is clear from our description that the dominant frequency here is the forcing frequency. Other frequencies which appear due to the nonlinearity will be shown, in section 5.4 , to be associated with the eddies we have observed on the western side of the basin. It looks as if large quasi-linear motion starts in the east and the western boundary generate nonlinearities as a response. Hence the nonlinearities seem concentrated in the west.

Turbulent Solution $\omega_{F_{n} . d .}=\mathbf{0 . 0 7 2 5}$ and $\left(\frac{\delta_{I}}{L}\right)_{W N}^{2}=\mathbf{0 . 0 1}$

To see how the solution we described above progresses as the nonlinearity increases, we have included in Figure B-15, a detailed sequence for the same wind forcing frequency at a wind forcing strength of $\left(\frac{\delta_{I}}{L}\right)_{W N}^{2}=0.01$. One of the most noticeable things is that there is negative vorticity permanently along the north wall of the basin, and positive vorticity permanently along the south wall of the basin. Starting in Figure B-15 (a), there is a patch of mostly negative vorticity occupying the top two thirds of the basin. The anticyclonic eddies in the middle of the basin propagate westward 
whilst the anticyclonic eddies against the north wall of the basin propagate eastward. This again is explained by the method of images. In this very turbulent solution, the anticyclonic eddies near the north wall are capable of propagating all the way to the eastern wall. In the solutions for $\left(\frac{\delta_{I}}{L}\right)_{W N}^{2}=0.006$ and $\left(\frac{\delta_{I}}{L}\right)_{W N}^{2}=0.008$, which are not so nonlinear, the anticyclonic eddies against the northern wall that are analogous to these eddies disappear before reaching the eastern wall.

In Figure B-15 (g) we observe that there is positive vorticity forming at the eastern wall in the lower two thirds of the basin. In Figure B-15 (g) to (1) this positive vorticity propagates to the west. Note that, for this solution, there is always negative vorticity at the north wall and positive vorticity at the south wall.

Inertial boundary layers form corresponding to the eddies piling up on the western, northern and southern walls. There are also interior boundary layers at times when eddies approach each other. This is apparent in Figures B-14 and B-15, where the stream lines are bunched together. Therefore, although the highest velocities will usually be found along the walls, there are still significant velocities in the interior.

To give an indication of the magnitude of the response, the maximum velocities are plotted against the nondimensional forcing frequencies in Figure B-16 for each set of runs.

\subsection{Analysis of Results}

Figure B-17 (a), (b), (c), (d), (e) and (f)is the Fourier transform over 300 days of the zonal velocity at the point $800 \mathrm{~km}$ east and $800 \mathrm{~km}$ north in the lower western half of the basin of the solutions forced at a frequency $\omega_{F_{n . d} .}=0.0725$ and increasing forcing strengths. In the weakly nonlinear solutions in Figures B-17 (a) and (b), the only frequencies present, other than the forcing frequency, are integral multiples of the forcing frequency. However, coinciding with the appearance of eddies in the turbulent solutions in Figure (c) (i.e. $\left(\frac{\delta_{I}}{L}\right)_{W N}^{2}=0.004$ ), we begin to observe many other frequencies which are not integral multiples of the forcing frequency. As the solution becomes more and more nonlinear, see Figures (d), (e), and (f), more frequencies 
appear and the magnitude of the terms oscillating at these frequencies increase. In every case the forcing frequency is the dominant frequency, as we would expect from our discussion in section 5.3 concerning Figures B-14 and B-15.

Figure B-18 shows the contour plots of the absolute value of the spatial coefficients from the Fourier time series (5.5).

$$
\Psi_{\text {TOTAL }}(x, y, t)=\sum_{l=0}^{300}\left(f_{l}(x, y) \mathrm{e}^{i \omega_{l} t}+f_{l}^{*}(x, y) \mathrm{e}^{-i \omega_{l} t}\right)
$$

where $l=0,1,2, \ldots, \omega_{l}=\frac{2 \pi l}{T}$ and $f_{l}(x, y)=\frac{1}{T} \int_{0}^{T} \Psi_{T O T A L}(x, y, t) \mathrm{e}^{i \omega_{l} t} d t$. Here, $T$ is the length of the integration which is 300 days.

The contours of the spatial coefficients corresponding to the terms which oscillate at frequencies that are integral multiples of the forcing frequency have been plotted. These frequencies are marked in Figure B-17. In Figures B-18 (a), (b), (c), (d), (e), and (f) we see that the dominant spatial structure corresponding to the frequency $\omega_{F n . d .}$ is the mode $(2,1)$. However, as the solution increases in nonlinearity, there are other modes contributing to this frequency. Likewise the spatial structures associated with the multiples of the forcing frequency have more then one mode present, and the number of modes present obviously increases as the forcing frequency increases. In the turbulent solutions, many modes are present for the spatial structures at higher frequencies and the dominant response is on the western side of the basin. This indicates that the frequencies other then the forcing frequency which appear in the solution are associated with the eddy motion on the western side of the basin.

Figures B-19 (a), (b), and (c), for the solution $\left(\frac{\delta_{I}}{L}\right)_{W N}^{2}=0.004$ and $\omega_{F_{n . d .}}=0.0725$, are the Fourier transform of the zonal velocity in the east, middle, and west of the basin, respectively. As we can see, there are more frequencies, especially higher frequencies, associated with the eddies in the west of the basin. This reinforces the prior observation regarding the spatial coefficients associated with non-forcing frequencies, which had a stronger response in the west basin.

\section{Explanation of Weakly Nonlinear Results}

Unfortunately, I do not have a thorough analysis to explain the frequencies which 
appear in the turbulent solution, where the nonlinear terms are dominant. We speculate that the ones which appear initially might be associated with a rational multiple of the forcing frequency or with the natural frequency of the system. Certainly, most of the frequencies which eventually appear will be caused by instabilities in the flow.

There is, however, a simple analysis to explain why frequencies which are multiples of the forcing frequency appear in the weakly nonlinear solutions, where the linear solutions are dominant.

As we know from equation (3.32), the particular linear solution oscillates only at the forcing frequency. Hence, after the transients have decayed the only frequency present will be the forcing frequency. If we include the nonlinear terms in our analysis of section 3.3 we see that frequencies other than the forcing frequency can be excited by the nonlinear terms. Substituting the series solution for the stream function (2.17) into the nonlinear $\mathrm{QG}$ equation and projecting the result onto a particular mode $(p, q)$ we obtain the following ordinary differential equation

$$
\frac{\partial A_{p q}}{\partial t}-\left(i \omega_{p q}-\gamma\right) A_{p q}=\frac{<\frac{\vec{\nabla} \times \vec{\tau}}{\rho H}: \Phi_{p q}>}{\left\langle\nabla^{2} \Phi_{p q}: \Phi_{p q}>\right.} \mathrm{e}^{i \omega_{F_{n . d .}} t}-\frac{\left\langle J(\Psi, \nabla \Psi): \Phi_{p q}\right\rangle}{\left\langle\nabla^{2} \Phi_{p q}: \Phi_{p q}\right\rangle}
$$

The nonlinear terms act as a forcing term along with the wind forcing. From the last term in equation (5.6) we see that the nonlinear terms will excite frequencies which are integral multiples of the forcing frequency. To understand this we first assume that the $\Psi$ in the Jacobian term is the single mode $\Psi_{m n}$ which dominates the weakly nonlinear solution at the forcing frequency. This is obviously an approximation to $\Psi$ which simplifies our analysis. Next we calculate $J\left(\Psi_{m n}, \nabla \Psi_{m n}\right)$, taking note that the frequency associated with the Jacobian is either $2 \omega_{F_{n . d}}$ or 0 . Considering equation (5.6), we see that if the Jacobian of $\Psi_{m n}$ projects onto a mode $\Psi_{p q}$ it will act as a forcing term for $A_{p q}$ and, hence, $A_{p q}$ will now have oscillations of $2 \omega_{F_{n . d} \text {. }}$ or have a steady component. Now we can approximate $\Psi$ as $\Psi_{m n} e^{\left(i \omega_{F_{n . d .}} t\right)}+\Psi_{p q} e^{\left(i 2 \omega_{F_{n . d .}} t\right)}+$ $\Psi_{p q}$. This is another approximation in that we are ignoring the excitation of other terms which project onto $J\left(\Psi_{m n}, \nabla \Psi_{m n}\right)$. If we repeat the argument and substitute $\Psi_{m n} e^{\left(i \omega_{F_{n . d .}}{ }^{t}\right)}+\Psi_{p q} e^{\left(i 2 \omega_{F_{n . d}} t\right)}+\Psi_{p q}$ into the Jacobian we see that frequencies of $\omega_{F_{n, d .}}$, 
$4 \omega_{F_{n . d .}}, 3 \omega_{F_{n . d .}}$, and 0 appear. Hence modes which project onto this Jacobian will be excited with these frequencies. The argument can be extended to show that all the frequencies which will be excited are multiples of the forcing frequency. Of course, this is exactly what we see for the weakly nonlinear solution.

We speculate that as the nonlinearity increases instabilities occur in the turbulent solution which excite frequencies that are not integer multiples of the forcing frequency.

\section{Scaling of Turbulent Results}

This turbulent problem is particularly difficult to scale. We have made a fairly accurate attempt to scale the weakly nonlinear problem far from the resonance in section 4.4 , and when we are in this regime $\left(\frac{\delta_{I}}{L}\right)_{W N}^{2}$ does in fact approximate the true Rossby number of the flow.

The reason why it is possible to have a reasonable scaling for the weakly nonlinear solution is that there is one length scale which is approximately the size of the basin (the solution is still dominated by a single mode). We know from the linear solution (3.32) that, when we are far from resonance, the time tendency of relative vorticity and the planetary vorticity term do not cancel very well and, therefore, we balance them with the forcing term. When the solution is at resonance these two terms do balance and then the forcing term balances the friction term.

However, when the solution transitions to the very nonlinear turbulent regime there are many time scales, length scales, and velocity scales present and, thus, it is much more difficult to decide which terms balance. In order to "see" how the balance changes after the transition, the time tendency of relative vorticity, the planetary vorticity term, the frictional term, and the Jacobian term of the QG equation have been calculated from the model results using a simple finite difference method. The meridional velocity was integrated to get the stream function and then the stream function was used to calculate the QG terms.

Figure B-20 shows the calculation of the QG terms for the solution $\left(\frac{\delta_{l}}{L}\right)_{W N}^{2}=$ 0.008 and $\omega_{F n \text {.d. }}=0.085$. This solution is still in the weakly nonlinear regime. As we can see, in the western side of the basin, where there is no forcing, the planetary 
vorticity term, Figure B-20 (a), and the time tendency term, Figure B-20 (b), are both of magnitude $10^{-11}$ and their contour plots complement each other. Hence we see that the main balance is between these two terms. The next biggest term is the Jacobian term, Figure B-20 (d), of magnitude $10^{-12}$. Note that this term is largest in the region of wind forcing, which might be introducing small length scales into the flow. Finally, the smallest term is the bottom friction term, which is of order $10^{-13}$, as shown in Figure B-20 (c).

Figure B-21 shows the calculation of the same terms for the solution $\left(\frac{\delta_{I}}{L}\right)_{W N}^{2}=$ 0.008 and $\omega_{F n \text {.d. }}=0.0825$. This time the main balance is between the time tendency of relative vorticity, Figure B-21 (b), and the Jacobian nonlinear term, Figure B-21 (d). The contour plots of these terms complement each other and they both are of order $10^{-10}$. This value is larger than in the previous case, due to the contribution of the small scales. Not surprisingly, these two terms, which have the most derivatives, contain the smallest scales of motion and balance each other in this regime. It is interesting to note that the sum of the Jacobian term and the time tendency term shows larger scale structures and balances the planetary vorticity term, which only has one derivative. The order of the planetary vorticity term, Figure B-21 (a), is $10^{-11}$. Again the friction term, Figure B-21 (c), is small and of order $10^{-12}$. Hence, we conclude that the main balance for the small scales of motion in the turbulent regime is between the time tendency and the Jacobian term. The balance for the larger scales of motion seems to be between the sum of the Jacobian term and the time tendency term and the planetary $\beta$ term.

The fact that our calculation of the QG terms from the shallow water model satisfy the $Q G$ equation indicates that we are in the $Q G$ regime for the solution $\left(\frac{\delta_{I}}{L}\right)_{W N}^{2}=0.008$ and $\omega_{F n . d .}=0.0825$.

\section{Hysteresis}

As we saw in section 5.3 there is a transition in the solution forced with the wind strength $\left(\frac{\delta_{I}}{L}\right)_{W N}^{2}=0.01$ between the forcing frequencies $\omega_{F_{n . d .}}=0.085$ and $\omega_{F_{n . d .}}=$ 0.0875 , see Figure B-11. These runs were forced with zero initial conditions.

We already know from previous work, see Pedlosky [3] and section 5.1.2, that 
the resonant peak leans towards higher frequencies as the nonlinearity in the weakly nonlinear solution increases, causing a hysteresis. It is interesting to see that the hysteresis is also present when the solution becomes turbulent. Figure B-22 shows the mean integrated kinetic energy of the original results with zero initial conditions, marked by a square, and the results of runs initialized by solutions forced at a lower frequency (moving from low to high), marked by asterisks. As we can see, the transition now occurs at the forcing frequency of $\omega_{F_{n . d} .}=0.0925$ as opposed to $\omega_{F_{n . d .}}=0.0875$ and there is indeed a hysteresis. These runs were done at our strongest forcing frequency of $\left(\frac{\delta_{I}}{L}\right)_{W N}^{2}=0.01$. When the runs are initialized by a higher forcing frequency (moving from high to low), once the runs have spun up, the runs are the same as when there are zero initial conditions and, hence, no hysteresis is found in that direction.

Figures B-23 (a) and (b) show the envelope plot of the runs before and after the transition. Figure B-23 (a) resembles the envelope contour before the transition, see Figure B-12 (d), with zero initial conditions and Figure B-23 (b) resembles the envelope contour after the transition, see Figure B-12 (d), with zero initial conditions. The transition is again from the turbulent solution to the weakly nonlinear solution.

We had observed, Pedlosky [3], for the weakly nonlinear solution that the interaction of the $\Psi_{0}$ and $\Psi_{1}$ solution caused a hysteresis in the amplitude curve of the solution. It is interesting to note that this hysteresis is still present when the solution transitions to its turbulent state when so many basin modes are present. 


\section{Chapter 6}

\section{Nonlinear Solution for the Barrier}

\section{Basin}

In our last chapter we will briefly consider the effect of the nonlinearity on the mode corresponding to $(2,1)$ in the barrier basin. Consulting Table A.1, we see that it has a nondimensional resonant frequency, 0.0686 , which is slightly less than the resonant frequency, 0.0712 , for the $(2,1)$ mode in the barrier-free basin.

\subsection{Numerical Results}

Our discussion centers on two sets of numerical runs forced with nondimensional frequencies ranging from 0.0625 to 0.085 . The forcing strength of the runs are $\left(\frac{\delta_{r}}{L}\right)_{W N}^{2}=0.004$ and 0.01 , which correspond to $2048\left[\mathrm{~cm}^{2} / \mathrm{s}^{2}\right]$ and $5120\left[\mathrm{~cm}^{2} / \mathrm{s}^{2}\right]$, respectively. All the other parameters remain the same.

\subsection{Description of Results}

Figure B-24 shows the mean integrated kinetic energy versus forcing frequency, $\omega_{F_{n . d} \text {. }}$, for both sets of runs, $\left(\frac{\delta_{I}}{L}\right)_{W N}^{2}=0.004$ and 0.01 .

The resonant peak has obviously been diminished, in amplitude and shape, by the nonlinearity. It is difficult to tell with the resulting broad peak and the frequency 
resolution of these runs if the nonlinearity has increased the natural frequency of the system from 0.0686 . To determine how the natural frequency has changed we could do an analysis similar to that of Pedlosky [3].

From Figure B-24 it is difficult to tell if there is a jump in the solution. Perhaps there might be one between the nondimensional forcing frequencies of 0.07 and 0.0725 for the solution $\left(\frac{\delta_{I}}{L}\right)_{W N}^{2}=0.004$, and between 0.075 and 0.0775 for the solution $\left(\frac{\delta_{I}}{L}\right)_{W N}^{2}=0.01$.

To help determine where the nature of the solution changes, we consider the envelope plots at each forcing frequency. Figures B-25 and B-26 present the contour plots for the forcing strengths $\left(\frac{\delta_{I}}{L}\right)_{W N}^{2}=0.004$ and $\left(\frac{\delta_{I}}{L}\right)_{W N}^{2}=0.01$, respectively. The envelope plots in Figure B-25 do not show any change in the nature of the solution. All solutions seem to be dominated by the linear solution in the eastern basin. The western basin seems to be more nonlinear for certain frequencies than others. However, there is no dramatic change from one forcing frequency to the next. Figures B-26 (a), (b), (c), (d), (e), (f), which plot the envelopes for the the solution forced at $\left(\frac{\delta_{I}}{L}\right)_{W N}^{2}=0.01$, on the other hand, seem to be strongly nonlinear throughout the basin for some frequencies. There is a slight change in the eastern basin between the solution in Figures B-26 (f) and (g). Figure B-26 (f), corresponding to a nonlinear frequency of $\omega_{F_{n . d .}}=0.075$, is nonlinear in the eastern basin whereas the solution in Figure B-26 (g), corresponding to a forcing frequency of $\omega_{F_{n, d}}=0.0775$, is clearly dominated by the linear solution in the eastern basin. Therefore, there does seem to be a "jump" of the type we observed in chapter 5 in the run $\left(\frac{\delta_{I}}{L}\right)_{W N}^{2}=0.01$. The question of whether there is an associated hysteresis will be addressed in section 6.3.

\section{Weakly Nonlinear vs Turbulent}

In chapter 5 we made a clear distinction between a weakly nonlinear solution and a turbulent solution. Part of this distinction was the fact that the only non forcing frequencies appearing in the weakly nonlinear solution were integral multiples of the forcing frequency, whereas many different frequencies appear in the turbulent solutions.

Now, in the barrier basin we have the odd situation in which the solution appears 
to be turbulent in one sub-basin and weakly nonlinear in the other sub-basin. It seems that, for this case, the non integral multiples of the forcing frequency which appear in the western sub-basin do have a signature in the eastern sub-basin. Hence, we have the situation in the eastern sub-basin where the solution is weakly nonlinear but there are frequencies present which are not integral multiples of the forcing frequency.

Solution $\omega_{F_{n . d .}}=0.0725$ and $\left(\frac{\delta_{I}}{L}\right)_{W N}^{2}=0.004$

We will now take a detailed look at how the solution $\left(\frac{\delta_{I}}{L}\right)_{W N}^{2}=0.004$ progresses in time. We have arbitrarily chosen the forcing frequency 0.0725 as in section 5.3. Figure B-27 indicates that this solution is dominated by the linear full basin mode from section 2.2 with the natural frequency 0.0686 . Most striking are the eddies formed at the tips of the barrier. When the flow about the barrier is clockwise (i.e. anticyclonic), corresponding to positive streamlines, these eddies are formed at the south-west and north-east of the barrier and also have a clockwise circulation. See Figures B-27 (b), (c), and (d). Note that the eddy formed in the west basin is much bigger and lasts much longer. Likewise, when the barrier is encircled by flow which is counterclockwise (i.e. cyclonic), corresponding to negative streamlines, eddies are formed at the north-west and south-west of the barrier. See Figures B-27 (h), (i), (j), and (k). Again, the eddy in the west is stronger and lasts longer than the eddy formed in the east. The anticyclonic eddy remains in the general area of the south of the western sub-basin.

This solution seems to be dominated by the full basin mode with natural frequency 0.0686. We have not speculated on what dynamics govern the eddies formed at the tips of the barrier.

Solution $\omega_{F_{n . d .}}=0.0725$ and $\left(\frac{\delta_{I}}{L}\right)_{W N}^{2}=0.01$

As we noticed from Figure B-26 (e) this solution seems to be turbulent throughout the whole basin. Figure B-28 shows the contour plots of the solution at two day intervals. In the same fashion as in the solution $\omega_{F_{n} . d .}=0.0725$ and $\left(\frac{\delta_{I}}{L}\right)_{W N}^{2}=0.004$, we again see eddies forming at the tips of the barrier, see Figures B-28 (a) and (b) (south-west and north-east of the barrier) and Figures B-28 (f), (d), (h), (i), (k), and (l) (north-west and south-east of the barrier). 
The anticyclonic eddy which forms at the south-west of the barrier seems to feed into the anticyclonic eddy that remains in the vicinity of the south of the west basin. According to the method of images, one would expect it to move northwards along the west basin meridional wall; we do see this in Figures B-28 (f), (g), and(h). Likewise, the cyclonic eddy at the north-west of the barrier feeds into the cyclonic eddy at the mid-latitude of the western basin. At times this cyclonic eddy moves south along the western basin meridional wall as predicted by the method of images, see Figures B-28 $(\mathrm{h}),(\mathrm{i}),(\mathrm{j})$, and $(\mathrm{k})$.

This solution seems to be more turbulent in the western basin. We would expect this from our results in chapter 5. Perhaps the small length scales found near the western wall are due to the reflection of eastward Rossby waves which have small length scales. This conclusion might lead one to ask why would we not have similar reflections from the meridional barrier and, hence, have small scale motions just to the east of the barrier in the eastern basin.

However, in this situation, with the barrier placed at $620 \mathrm{~km}$, we also have the introduction of small length scales by the small zonal scale of the west sub-basin and the small gap widths. One might wonder if it is these small length scales that introduce the small scale motions in the western basin.

To assess the influence of the small length scales of the barrier in producing the small scale motions observed in the western sub-basin, we have made the size of the eastern sub-basin the usual size of the western sub-basin and vise versa for the results shown in Figure B-29. As well as moving the barrier, the wind forcing was moved to the meridional strip between $200 \mathrm{~km}$ and $400 \mathrm{~km}$, thereby producing a problem which is the east-west mirror image of the problem in Figure B-28. Hence, if the small scale motions observed in the western sub-basin of Figure B-28 where indeed only caused by the small scales due to the position of the barrier, then one might expect similar small scale motions to appear in the eastern sub-basin in Figures B-29. The absence of such motions indicate that the small scales were perhaps caused by the reflection of small wavelength eastward propagating Rossby waves from the western basin meridional wall. 
The above conclusion leads us to wonder then why do not we see similar small scale motions to the east of the barrier in Figures B-28. One explanation might be that the reflection from the barrier, which must satisfy the no net circulation condition, might be different than the reflection at a wall of the basin.

\subsection{Analysis of Results}

We will now try to make a more quantitative analysis of the results.

\section{Frequencies Excited by Nonlinearities}

Figure B-30 shows the Fourier Transform of the zonal velocity at different points in the basin for forcing strengths $\left(\frac{\delta_{I}}{L}\right)_{W N}^{2}=0.004$ and $\left(\frac{\delta_{I}}{L}\right)_{W N}^{2}=0.01$ and forcing frequency 0.0725 . The Fourier transform is calculated over 300 days and, therefore, the accuracy of the peaks in the Fourier transform in Figure B-30 is 0.0061.

For the solution $\left(\frac{\delta_{I}}{L}\right)_{W N}^{2}=0.01$ and $\omega_{F_{n . d .}}=0.0725$ the dominant frequency is the forcing frequency at which the energy is put into the system. Most of the energy is then scattered into the lower frequencies, as shown in Figures B-30 (a), (c), and (e). There are so many different frequencies present that it is difficult to distinguish between them.

In contrast, Figure B-30 (b), (d), and (f) show that there are a select few frequencies present for the solution $\left(\frac{\delta_{r}}{L}\right)_{W N}^{2}=0.004$ and $\omega_{F_{n . d} .}=0.0725$. These are labeled 1 , $2,3,4,5,6,7$, and 8 . As we expect from section 5.4, some of these frequencies, peaks $4,6,7$, and 8, are associated with integer multiples of the forcing frequency. The other peaks, 1,3 , and 5 , are the frequencies which arise when a solution transitions to its turbulent state. The appearance of these non-integer multiples of the forcing frequency in the solution $\left(\frac{\delta_{I}}{L}\right)_{W N}^{2}=0.004$ and $\omega_{F_{n . d .}}=0.0725$ indicate the solution is transitioning to its turbulent state.

As mentioned in chapter 5 , we have no explanation of how these frequencies arise and what their relationship is to the forcing frequency and the natural frequencies of the system. In this particular example the frequency of peak 1 is very close to half the forcing frequency, peak 3 is close to three halves the forcing frequency and peak 5 
is close to five halves the forcing frequency. Could it be possible that these extra frequencies are associated to rational multiples of the forcing frequency? Alternatively, peak 1 also has a frequency which is close to the natural frequency of the first western sub-basin mode and the natural frequency of the fifth full basin mode, and peak 3 has a frequency close to the natural frequency of the first full basin mode, see Table A.1. Hence, peak 5 could result as the sum of the frequencies of peaks 2 and 3 . This is only speculation.

Figure B-31 shows the Fourier transform of the zonal velocity at a point for the forcing frequency $\omega_{F_{n . d}}=0.0725$ and increasing forcing strengths up to $\left(\frac{\delta_{I}}{L}\right)_{W N}^{2}=$ 0.004. The indication here is that the first frequency that is not a multiple of the forcing frequency to appear is the peak 3 .

Figure B-32 shows the spatial coefficients associated with the frequencies of peaks $1,2,3,4,5,6,7$, and 8 (note that there is also a peak associated with the steady circulation and is not labeled on diagram). Most prominent is the second full basin mode with resonant frequency 0.0686 associated with the forcing frequency. Also apparent is the antisymmetric first eastern sub-basin mode associated to twice the forcing frequency, and the antisymmetric structure associated with the steady part of the solution. We will explain in the next section why antisymmetry about the mid-latitude is associated to an even multiple of the forcing frequency.

None of the basin modes corresponding to the natural frequencies which we associated with the peaks 1 and 3 seem to be present. This may be because, as well as needing a forcing at the natural frequency, we also require the correct spatial structure. Perhaps the spatial structure of the forcing has small projection on the basin modes associated with peaks 1 and 3 .

Also of interest in the spatial structures of the time Fourier coefficients is the fact that most of these other frequencies are associated with the eddies at the tip of the barrier. This is analogous to Figure B-18 in section 5.4 where we found that the frequencies for the nonlinear solutions where associated with the eddies which formed in the west basin. Oddly, the eddies in the south of the basin are associated to the frequencies at peak 1 and 3 but the eddies in the top do not have a similar connection. 
This may be a manifestation of the asymmetry of the shallow water equations about the mid-latitude.

\section{Another Look at the QG-Equation}

Now we return to equation (5.6) and use it to predict the weakly nonlinear solution for the case of both antisymmetric meridional forcing and symmetric meridional forcing.

In order to speculate about the weakly nonlinear solution of the barrier problem, we consider the Jacobian term and how it will project onto different modes, thereby forcing these modes. Therefore, let us consider the terms in the Jacobian in greater detail

$$
\frac{\partial \Phi}{\partial x} \frac{\partial \nabla^{2} \Phi}{\partial y}-\frac{\partial \Phi}{\partial y} \frac{\partial \nabla^{2} \Phi}{\partial x}
$$

In particular, we are interested in the meridional structure of the stream function which is always expressed in terms of $\sin (n \pi y)$ 's, for $n=1,2,3, \ldots$ For a sub-basin mode it would be a single $\sin (n \pi y)$ with $n$ even; for a full basin mode it would be a summation of $\sin (n \pi y)$ with odd $n$ 's. For the moment, we will consider the Jacobian action on a single $\sin (n \pi y)$. The Laplacian, which has a double $y$-derivative, will not change the symmetry of the function. However the single $y$-derivative in the Jacobian will. A typical Jacobian term will be of the form

$$
A(x) \sin (p \pi y) B(x) \cos (q \pi y)=\frac{A(x) B(x)}{2}(\sin ((p+q) \pi y)+\sin ((p-q) \pi y))
$$

Thus, (6.2) brings out our main point: if $p$ and $q$ are both even, or both odd, then $p+q$ and $p-q$ will both be even. Therefore, whenever we take the Jacobian of two symmetric, or two antisymmetric, modes the result is always antisymmetric about the mid-latitude. Likewise, if exactly one of $p$ and $q$ is even (and hence the other odd) then $p+q$ and $p-q$ will both be odd. Hence, the Jacobian would project only on a symmetric mode about the mid-latitude.

In summary, the Jacobian of two full basin, or sub-basin, modes projects only onto sub-basin modes and the Jacobian of one full basin and one sub-basin mode projects only onto full basin modes.

We have seen in our analysis of the barrier-free problem that when our solution 
is weakly nonlinear one of the first effects is that frequencies equal to $m \omega_{F}$ appear, where $m$ is an integer. For example, when we take the Jacobian of two terms which both oscillate at the forcing frequency $\omega_{F}$, the modes for which this Jacobian projects onto will then oscillate at the frequencies 0 and $2 \omega_{F}$. Extending this reasoning, we obtain many terms which oscillate at frequencies which are multiples of the forcing frequencies.

We conclude that if we force the basin with a forcing which is purely symmetric about the mid-latitude then the terms in our solution, see (2.17), which are full basin modes should oscillate at frequencies that are odd multiples of the forcing frequency. Likewise, terms in (2.17) which oscillate at frequencies which are even multiples of the forcing frequency should be sub-basin modes.

The conclusions above have some interesting consequences. For example, if we consider the steady circulation, we see that, whether the forcing is symmetric or antisymmetric about the mid-latitude, the steady circulation will always be associated with sub-basin modes. This implies that there will never be a steady circulation through the gaps in the periodically forced solution. If we consider the circulation condition, it is very difficult to envision a steady circulation which is able to go through the gaps and still satisfy the no circulation condition about the barrier.

These hypotheses have been confirmed by running the model with forcings which are symmetric and antisymmetric about the mid-latitude of the basin. Figure B33 (a) is the Fourier transform of the zonal velocity of the solution forced at the frequency $\omega_{F_{n . d .}}=0.0725$ and forcing strength $\left(\frac{\delta_{I}}{L}\right)_{W N}^{2}=0.002$. The meridional structure of the forcing is symmetric. As expected, the frequencies which appear are integer multiples of the forcing frequency. The peaks have been labeled in the same fashion as in Figure B-30 (b). Figures B-33 (b), (c), (d), and (e) are the contours of the absolute value of the spatial coefficients associated which these frequencies. As predicted, the coefficients associated with the even multiples of the forcing frequency are antisymmetric about the mid-latitude and the coefficients associated with the odd multiples of the forcing frequency are symmetric about the mid-latitude.

Similarly, Figure B-34 (a) is the Fourier transform of the zonal velocity of the 
solution forced at the frequency $\omega_{F_{n . d .}}=0.0725$ and forcing strength $\left(\frac{\delta_{I}}{L}\right)_{W N}^{2}=0.002$. The meridional structure of the forcing is antisymmetric. Again the frequencies which appear are integer multiples of the forcing frequency. The peaks have been labeled accordingly to their relation with the forcing frequency. Figures B-34 (b), (c), and (d) are the contours of the absolute value of the spatial coefficients associated with these frequencies. As predicted, all the coefficients are antisymmetric. The result in Figure B-34 (b) is a little unexpected in that there are two jets exiting and returning to the eastern sub-basin. This is something we have not previously observed. It might be a non-QG effect appearing in the shallow water model in the regime of the small gaps or perhaps it might be that the antisymmetric QG modes really do have such features. Our earlier analytical work of chapter 3 would not have predicted such features since we made an assumption of uniform flow through the gap. It may be possible that, for these small length scales, the uniform flow through the gap assumption breaks down.

\section{Hysteresis}

As mentioned at the beginning of this chapter, the runs with a forcing strength of $\left(\frac{\delta_{I}}{L}\right)_{W N}^{2}=0.01$ indicate that there might be a possible hysteresis between the solutions forced at $\omega_{F_{n . d .}}=0.075$ and $\omega_{F_{n . d .}}=0.0775$. A preliminary investigation, of the type carried out for Figure B-22, to look for this possible hysteresis was conducted by initializing a run at a forcing frequency of $\omega_{F_{n . d .}}=0.0775$ with the results from the run forced at $\omega_{F_{n . d .}}=0.075$ with zero initial conditions. No hysteresis was found.

Another attempt was made to find a possible hysteresis at these forcing frequencies of $\omega_{F_{n . d .}}=0.075$ and 0.0775 and strength $\left(\frac{\delta_{I}}{L}\right)_{W N}^{2}=0.01$ for a regime of small bottom frictions, $\frac{\delta_{S}}{L}=0.001$. Figure B-36 plots the integrated kinetic energy of these runs vs the forcing frequencies. The zero initial condition runs are marked by asterisks $\left({ }^{*}\right)$ and the run $\omega_{F_{n . d .}}=0.0775$ initialized with the solution from $\omega_{F_{n . d}}=0.075$ is marked by a circle (o). As can be seen from Figure B-36, these two solution have different energy levels, indicating that we have found a hysteresis. Figure B-37 plots the integrated kinetic energy versus time for the two solutions forced at $\omega_{F_{n . d} .}=0.0775$. The dotted line indicates the solution was initialized with zero initial conditions and the solid line indicates the solution was initialized with the solution from $\omega_{F_{n . d .}}=0.075$. As we can 
see, the kinetic energy for the zero initialized solution is consistently lower, assuring us that Figure B-36 is an accurate representation of their relative kinetic energies. Finally, Figure B-38 contours the envelope functions for these two solutions. Clearly, Figure B-38 (a) (the zero condition initialized solution) is in a more linear regime than Figure B-38 (b) (the non-zero initialized solution).

All indications are that we have found a similar hysteresis to that in section 5.4 for the barrier free solution. The hysteresis seems to correspond to the solution transitioning to a turbulent solution in the eastern sub-basin. It would be interesting to see if there is another hysteresis for the barrier problem corresponding to the solution transitioning to a turbulent solution in the western basin. To search for this one would have to force the solution at a lower forcing strength, starting with a solution which was weakly linear throughout the basin and then look for a transition to turbulence in the western basin. 


\section{Chapter 7}

\section{Conclusions}

We have examined the linear and nonlinear steady periodic forced problems of basins both with and without a meridional barrier.

When the barrier was included we were primarily interested in how an eastern sub-basin forcing could produce a strong response in the western sub-basin when there were only two very narrow gaps for communication between the sub-basins. We saw that the strong western sub-basin response was due to the necessity of satisfying the circulation condition, which was simply an application of Kelvin's theorem about the barrier. However, we also found it was impossible to excite purely antisymmetric western sub-basin modes when the forcing was contained in the eastern sub-basin. Interestingly, we found some symmetric full basin modes which had features of western and eastern sub-basin modes.

Quite a bit of time was spent examining the effects of a symmetric forcing versus an antisymmetric forcing about the mid-latitude. Full basin modes were symmetric about the mid-latitude and could not satisfy the circulation condition on only one side of the barrier, whereas sub-basin modes were antisymmetric about the barrier and could satisfy the circulation condition on only one side of the barrier. Therefore, in the linear problem, only when the forcing had a component which was symmetric about the mid-latitude was there any western sub-basin response and, in addition, if the forcing frequency was near a full basin eigenfrequency there would be a very strong western sub-basin response. 
We also observed that there is a subset of symmetric sub-basin modes in our original set of full basin modes in section 2.2. This is a result that would have been difficult to predict by only considering the formulas in chapter 2 (from Pedlosky and Spall [4]). From these formulas, it is quite clear that there are a set of symmetric full basin modes and a set of antisymmetric sub-basin modes. However, it is not at all obvious that, within the set of symmetric full basin modes, there is a subset of modes whose response is dominant in only one sub-basin. The subset of the symmetric subbasin modes differ from the anti-symmetric sub-basin modes in that they require a small response in the opposing basin to satisfy the no net circulation condition. The antisymmetric modes, on the other hand, have absolutely no response in the opposing basin.

Interestingly, the subset of symmetric sub-basin modes are analogous in structure and frequency to symmetric modes of a basin the size of the sub-basin. This observation had already been made for the antisymmetric sub-basin modes. One might be led to believe that all of the modes for the barrier basin were analogous to modes for the barrier-free basin. However, we have found at least two full modes which are not analogous to any barrier-free modes.

The effects of bottom friction were also examined in the linear problem. Surprisingly, it was found that when the friction increased the response of the system at an off resonant frequency could actually increase. This occurs because although the resonant peaks are diminishing in magnitude they become wider and merge together, and so actually increase the response at frequencies in between.

After a quick review of some previous work carried out on the weakly nonlinear solution for the barrier-free basin, see [2] and [3], we examined the hysteresis of the resonant peak as the nonlinearity of the problem was increased for the barrier-free basin problem. Bottom friction remained small. It was observed that once the peak folded there was a "jump" to a turbulent solution which was dependent on the forcing frequency and the forcing strength.

As in the weakly nonlinear solution, a hysteresis was found for the very nonlinear runs. Model runs were conducted both with zero initial conditions and with the 
results of runs forced with a lower frequency. The runs which were initialized with the lower forcing frequency run remained nonlinear at higher frequencies than the runs with the zero initial conditions and, hence, a hysteresis was found. It is surprising that the hysteresis which is present in the weakly nonlinear solution remains when the solution transitions to its turbulent state. It would be interesting to see if the dynamics of this hysteresis is the same for both the weakly nonlinear and turbulent cases.

Finally, we examined the nonlinear solutions to the barrier basin. To begin with we concentrated on the more predictable weakly nonlinear solution. It was predicted and confirmed that, regardless of the symmetry of the forcing structure, the steady component of the solution (as well as any even multiple of the forcing frequency) would always project onto the antisymmetric sub-basin modes. This result is quite interesting and, most importantly, tells us that there is never any steady net flow through the gaps of the barrier in this periodically forced problem. Of course, considering the no net circulation condition and the rigid lid of the model, it would be hard to imagine how one could have a steady flow from one sub-basin to the other.

A hysteresis was observed for the forcing $\left(\frac{\delta_{I}}{L}\right)_{W N}^{2}=0.01$ between the forcing frequencies of $\omega_{F_{n . d}}=0.075$ and 0.0775 for the barrier basin. In order to see this it was necessary to reduce the bottom friction for the problem. This hysteresis seems to correspond to a transition between a weakly nonlinear state for the eastern sub-basin and a turbulent state for the eastern sub-basin. During this transition the western sub-basin seems to be continually in a turbulent state. It would be interesting to see if a similar hysteresis corresponds to a transition between a weakly nonlinear state in the western sub-basin and a turbulent state for the western sub-basin.

We also considered the situation in which the forcing is on the west side of the basin and the barrier is placed on the east side of the basin. This was done to see if the small scales of motion observed in the western sub-basin might be connected to the small length scales induced by the gaps and the small size of the western subbasin. Again, the small scales of motion were observed in the west indicating that, perhaps, they are due to the reflection of small scale eastward propagation Rossby 
waves. Surprisingly, no small scale motions were observed to the east of the barrier, suggesting that the reflection of the eastward Rossby waves differs somehow on the barrier than on the basin wall. It would be interesting to check observations off a long meridional coast, such as Japan, to see if there are fewer eddies there than one would expect off a western boundary, such as the east coast of Asia. 
Appendix A

Tables 


\begin{tabular}{|c|c|}
\hline Barrier Basin & Barrier Free Basin \\
\hline $1^{\text {st }}$ Full Basin & $\mathrm{m}=1, \mathrm{n}=1$ \\
$\omega=0.1057$ & $\omega=0.1125$ \\
\hline $2^{\text {nd }}$ Full Basin & $\mathrm{m}=2, \mathrm{n}=1$ \\
$\omega=0.0686$ & $\omega=0.0712$ \\
\hline $1^{\text {st }}$ Eastern Sub-basin & $\mathrm{m}=1, \mathrm{n}=2$ \\
$\omega=0.0648$ & $\omega=0.0648$ \\
\hline $3^{\text {rd }}$ Full Basin & $\mathrm{m}=3, \mathrm{n}=1$ \\
$\omega=0.0503$ & $\omega=0.0503$ \\
\hline $2^{\text {nd }}$ "Eastern" Sub-basin & $\mathrm{m}=1, \mathrm{n}=3$ \\
$\omega=0.0479$ & $\omega=0.0479$ \\
\hline $3^{\text {rd }}$ Eastern Sub-basin & $\mathrm{m}=2, \mathrm{n}=2$ \\
$\omega=0.0456$ & $\omega=0.0456$ \\
\hline $4^{\text {th }}$ Full Basin & \\
$\omega=0.039$ & \\
\hline $5^{\text {th }}$ Full Basin & \\
$\omega=0.0381$ & $\mathrm{~m}=1, \mathrm{n}=3$ \\
\hline $1^{\text {st }}$ "Western" Sub-basin & $\omega=0.0356$ \\
$\omega=0.0356$ & \\
\hline
\end{tabular}

Table A.1: Comparison of the nine resonant solutions in Figures B-5 and B-6 to the normal modes for a barrier-free basin to which they resemble. The resemblance is observed both in the structure of the solution and in the frequencies. For the case where the dominant response is in a sub-basin we consider a basin the same size. The quotation marks indicate that a sub-basin solution is symmetric and hence is part of the subset of full basin modes and not a part of the set of antisymmetric sub-basin modes. 
Appendix B

\section{Figures}




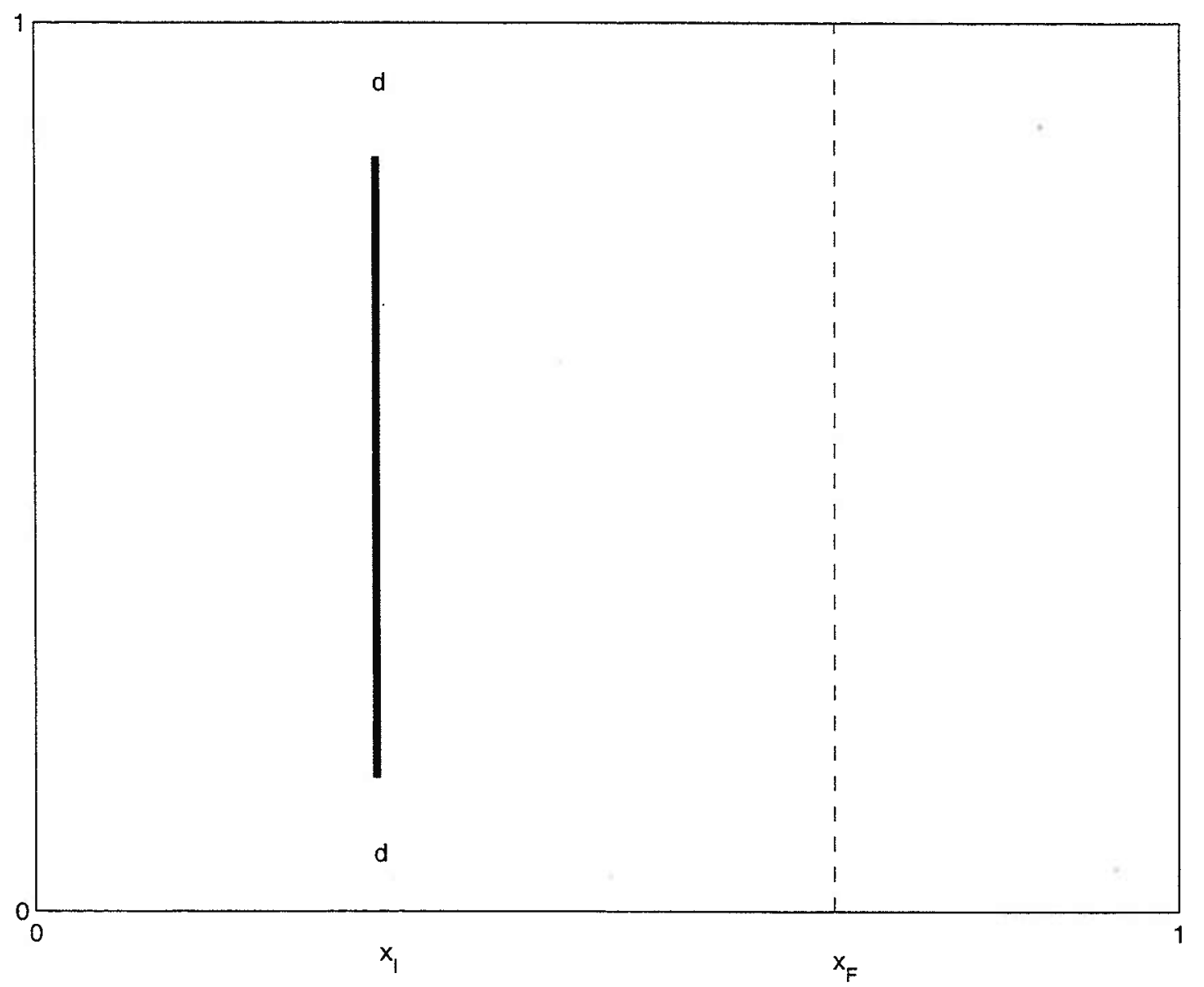

Figure B-1: Sketch of the square barrier basin with the meridional barrier placed at $x_{I}$ and the forcing along the dotted line at $x_{F}$. There are two gaps of width $d$ to the north and south of the barrier. 
(a)

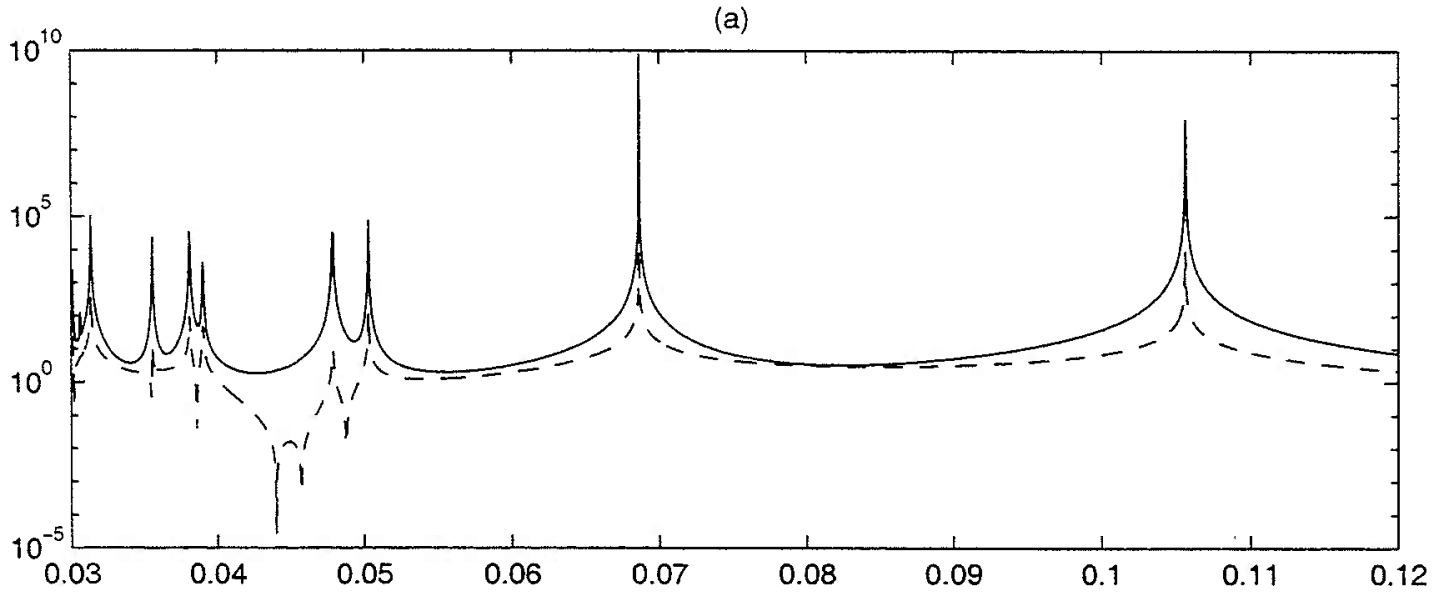

(b)

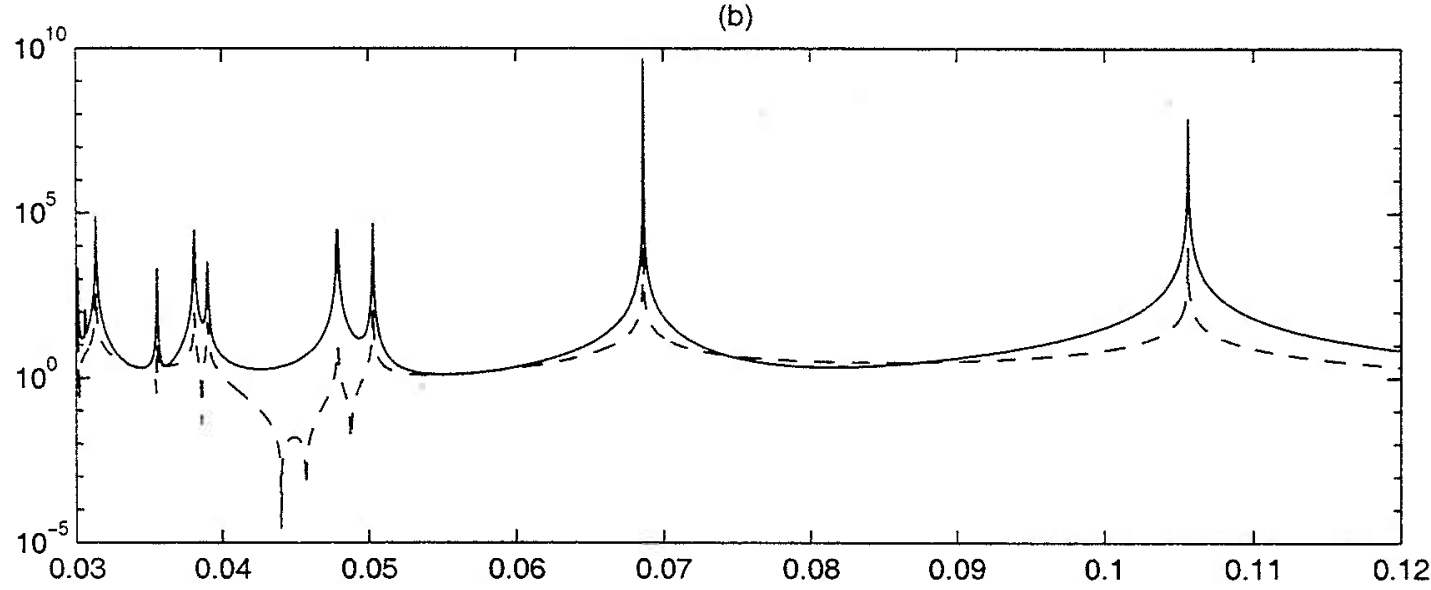

(c)

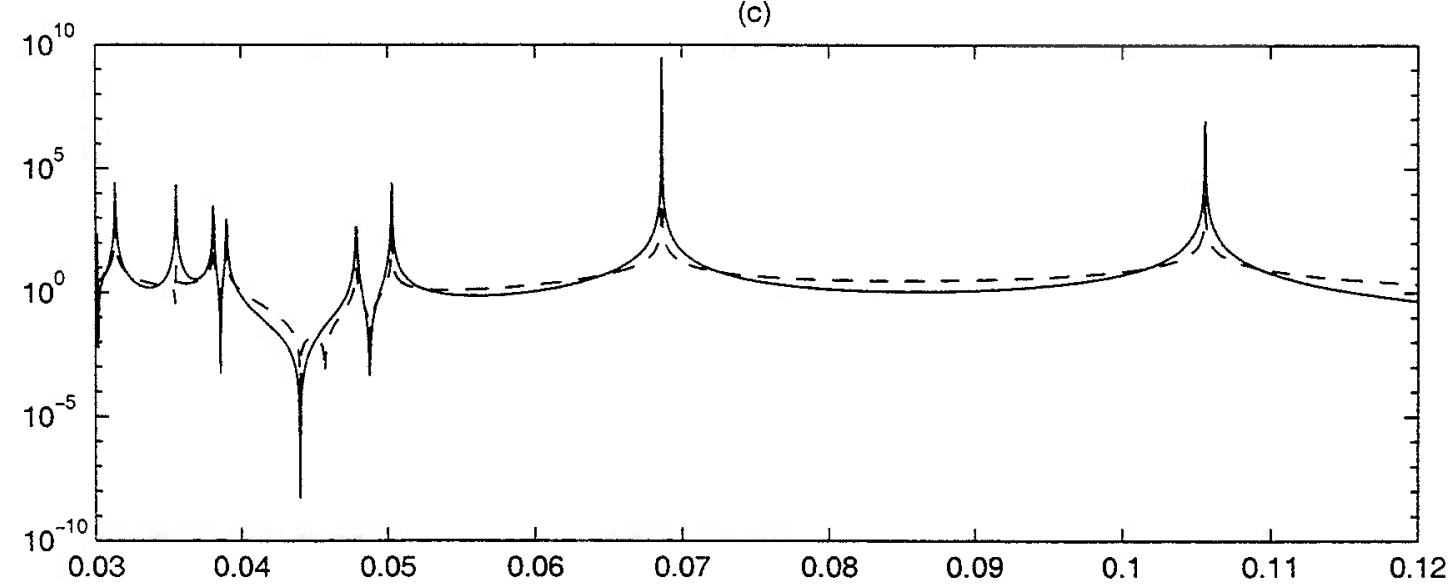

Figure B-2: Semilog plots of $\left|\phi_{I}\right|$ (- - ) and (a) full basin variance, (b) eastern subbasin variance and (c) western sub-basin variance vs forcing frequency $\omega_{F_{n . d}}$ for the case where $x_{I}=0.3, x_{F}=0.7, d=0.05, \gamma=10^{-8}$ and the meridional structure of the forcing is symmetric about $y=0.5$. 
(a)

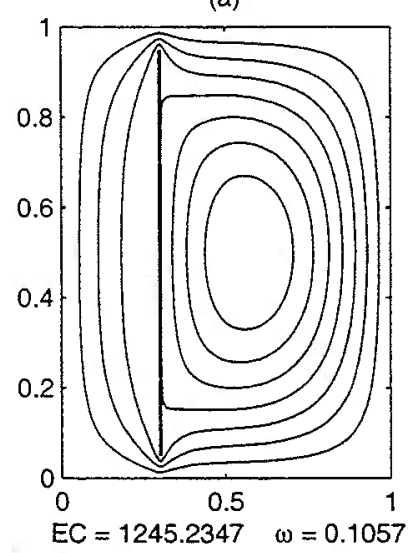

(d)

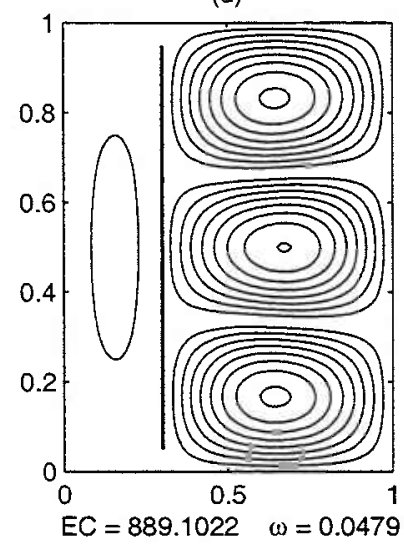

(g)

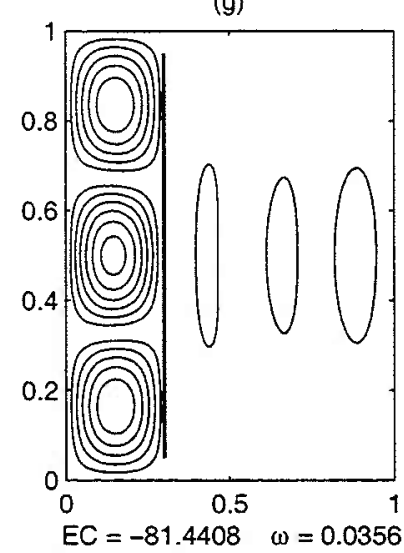

(b)

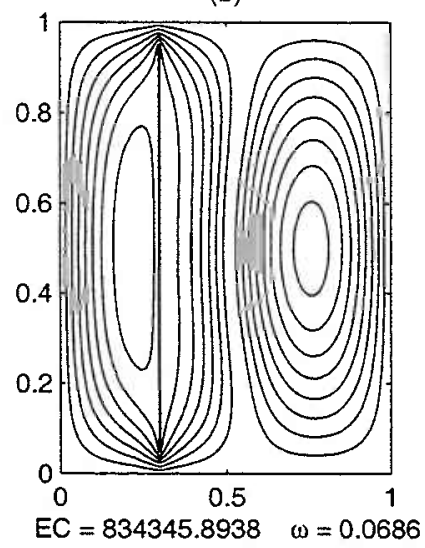

(e)

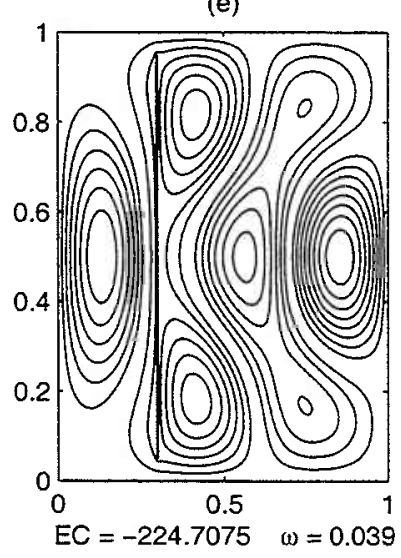

(c)
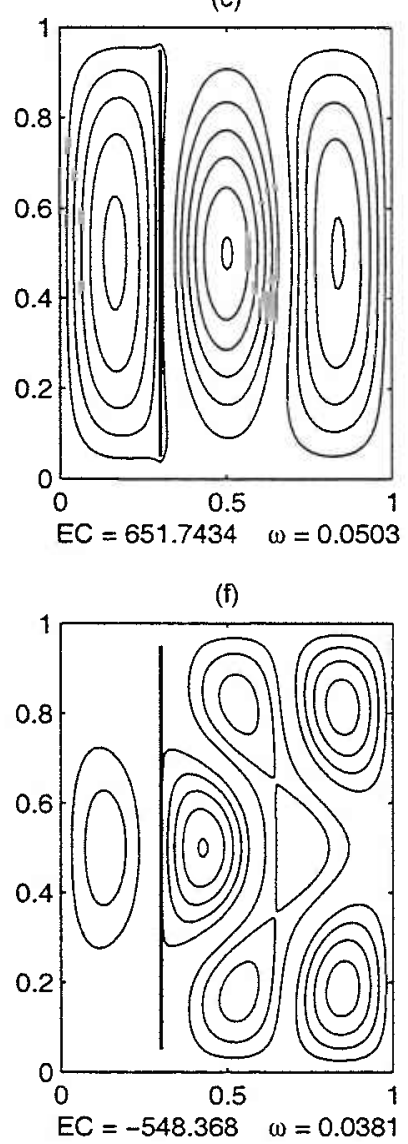

Figure B-3: Contour plots of the absolute value of the envelope function, $|\phi(x, y)|$, for the case where $x_{I}=0.3, x_{F}=0.7, d=0.05, \gamma=10^{-8}$, and the meridional structure of the forcing is symmetric about $y=0.5$. The forcing frequencies, shown under each plot, correspond to the first 7 peaks in the full basin variance graph in Figure B-2 (a). The value EC shown to the left of each forcing frequency is the circulation on the eastern side of the barrier (see appendix $\mathrm{C}$ for calculation of $\mathrm{EC}$ ) . 
(a)

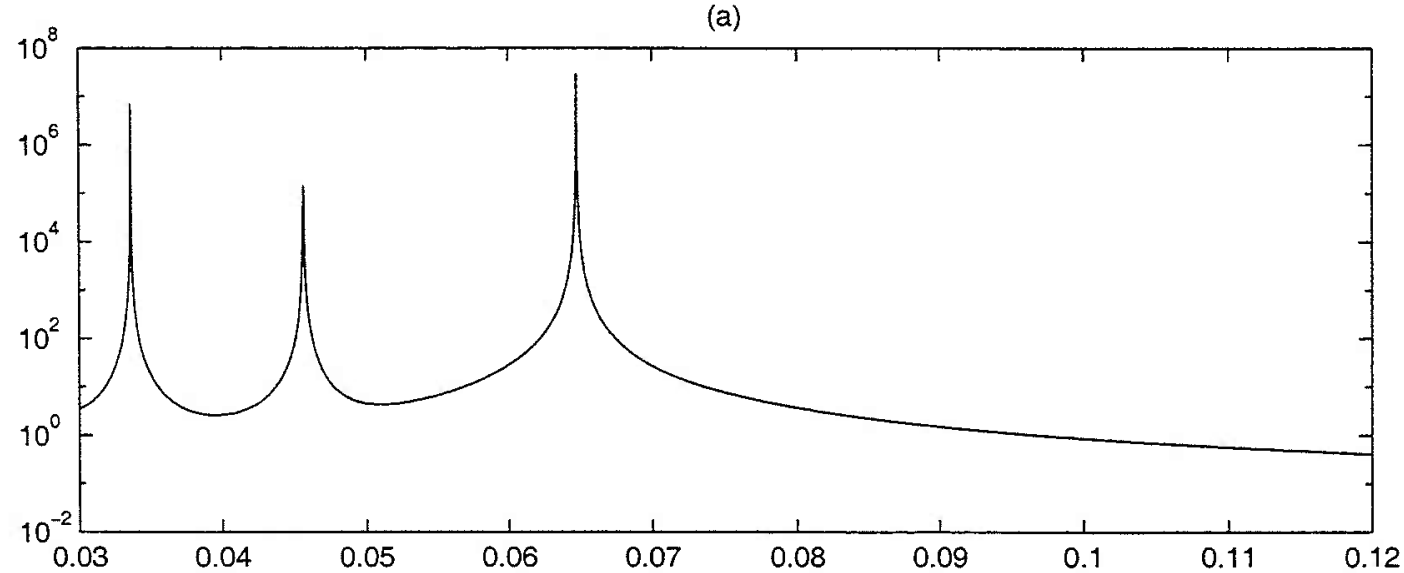

(b)

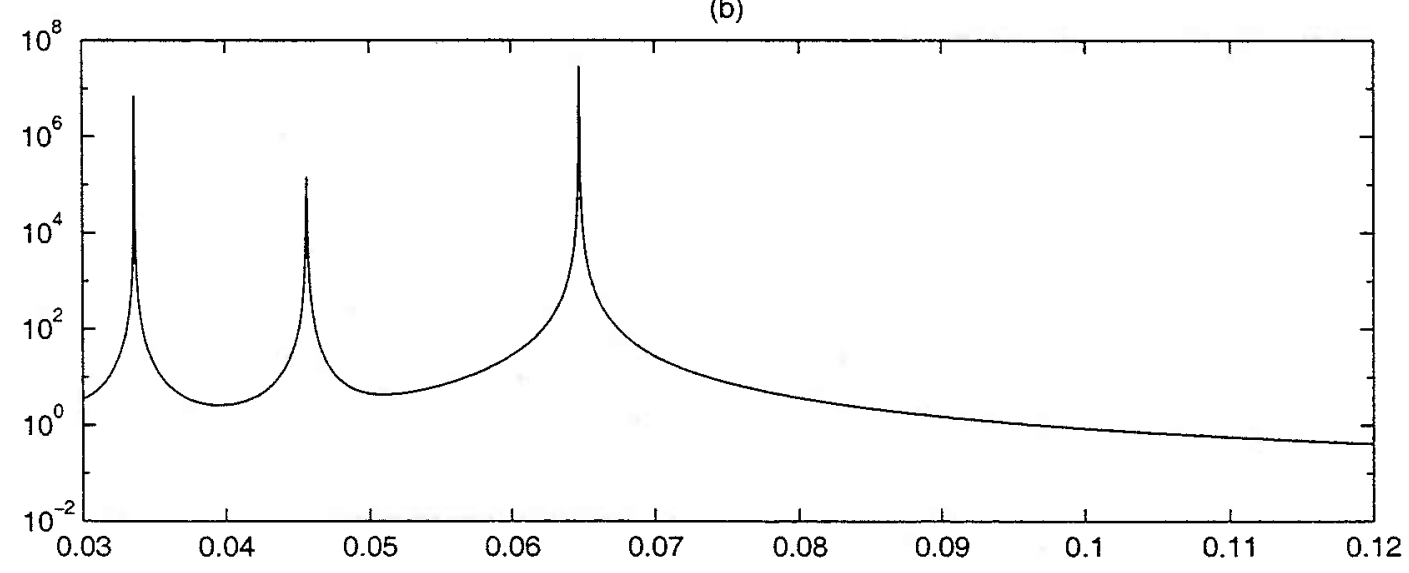

(c)

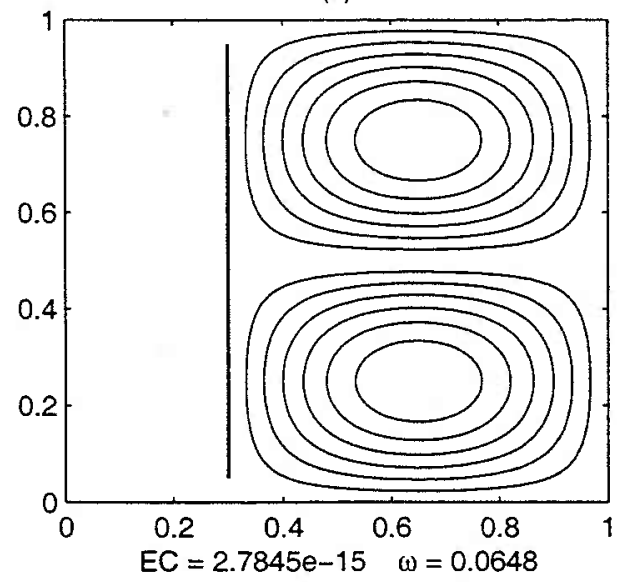

(d)

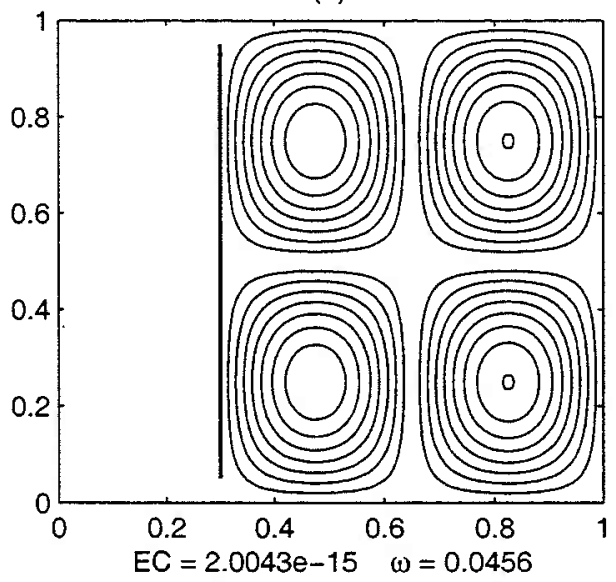

Figure B-4: Semilog plots of (a) full basin variance, (b) eastern sub-basin variance vs forcing frequency $\omega_{F_{n . d}}$. Plots (c) and (d) are contours of the absolute value of the envelope function, $|\phi(x, y)|$, at frequencies corresponding to the first two peaks of the full variance. $x_{I}=0.3, x_{F}=0.7, d=0.05, \gamma=10^{-8}$ and antisymmetric meridional structure of the forcing about $y=0.5$. 
(a)

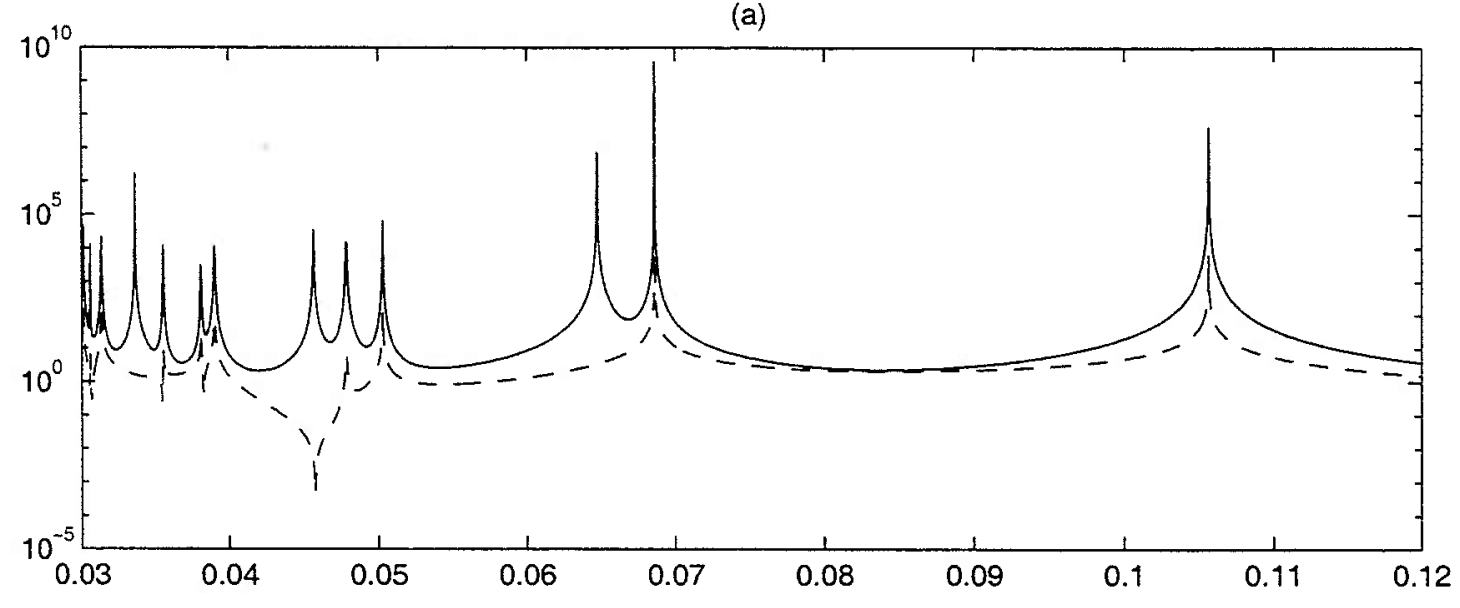

(b)

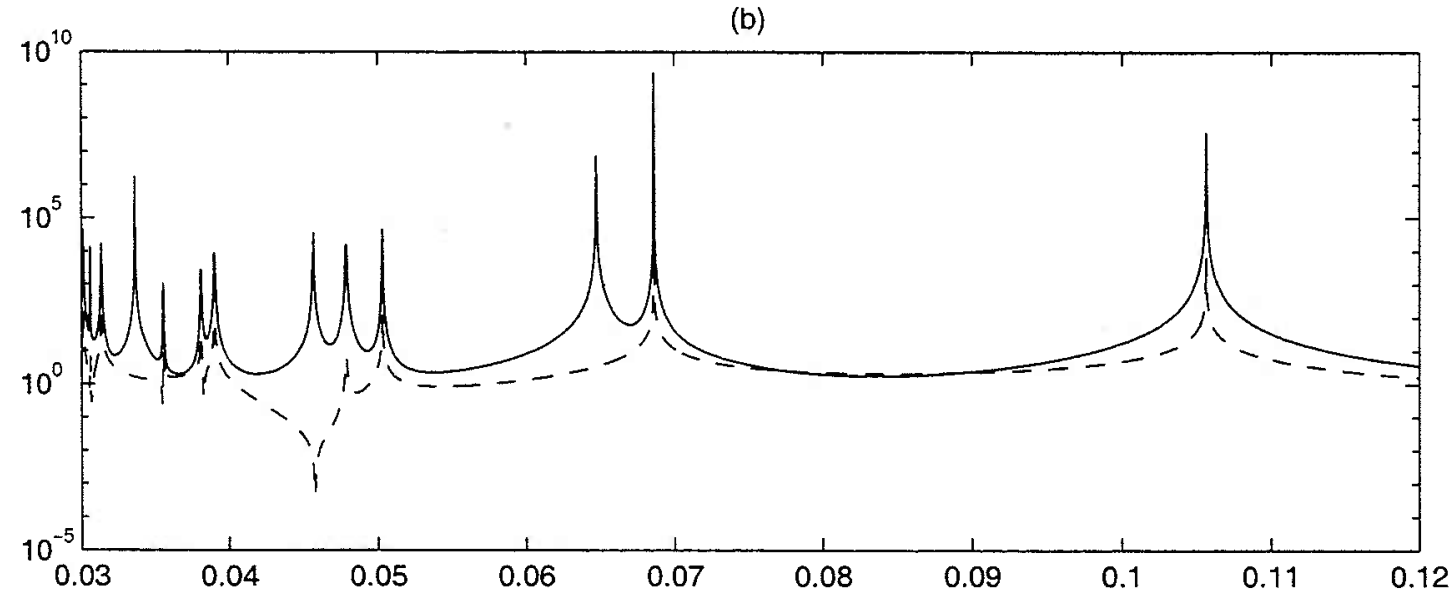

(c)

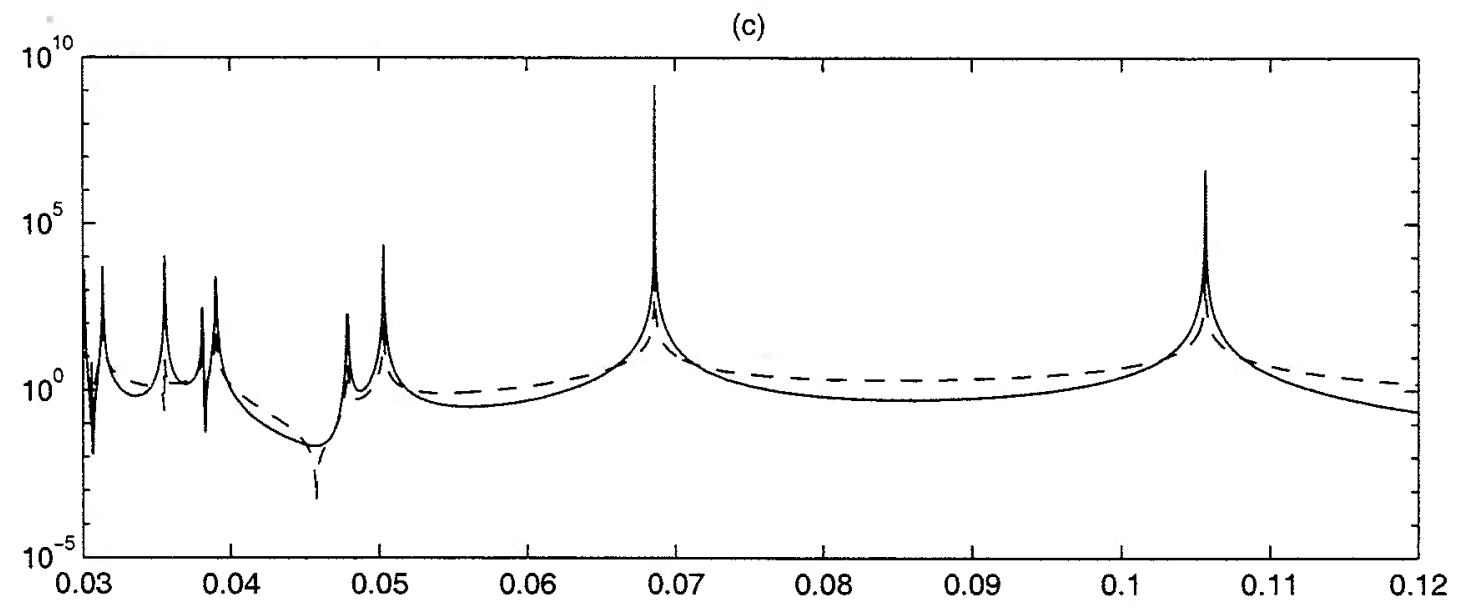

Figure B-5: Semilog plots of $\left|\phi_{I}\right|(--)$ and (a) full basin variance, (b) eastern sub-basin variance and $(c)$ western sub-basin variance vs forcing frequency $\omega_{F_{n . d .}}$ for $x_{I}=0.3, x_{F}=0.7, d=0.05, \gamma=10^{-8}$, and the asymmetric meridional structure of the forcing. 
(a)

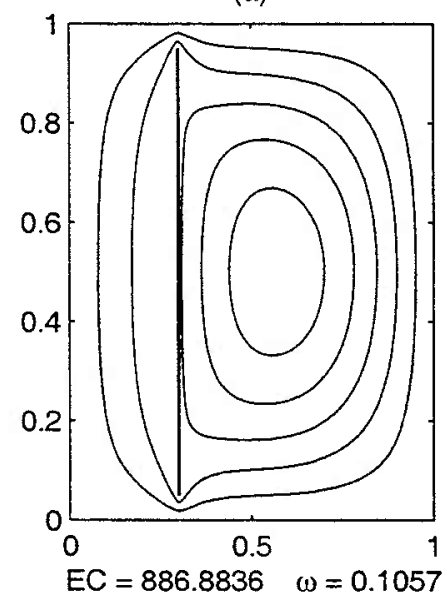

(d)

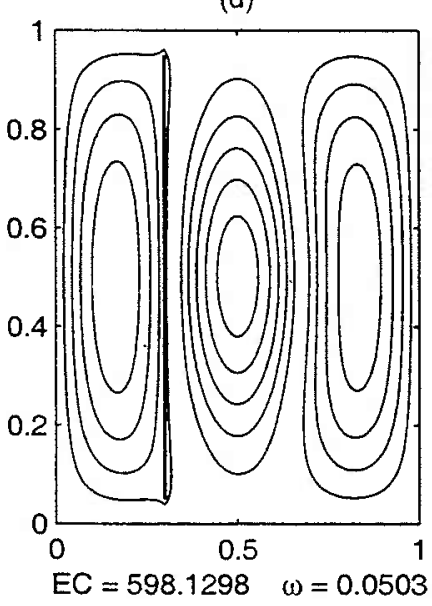

(g)

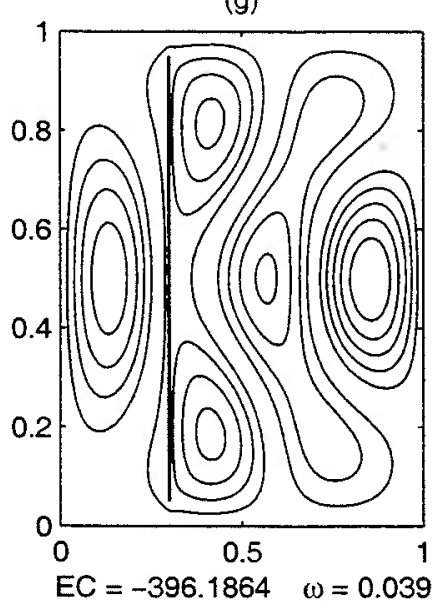

(b)

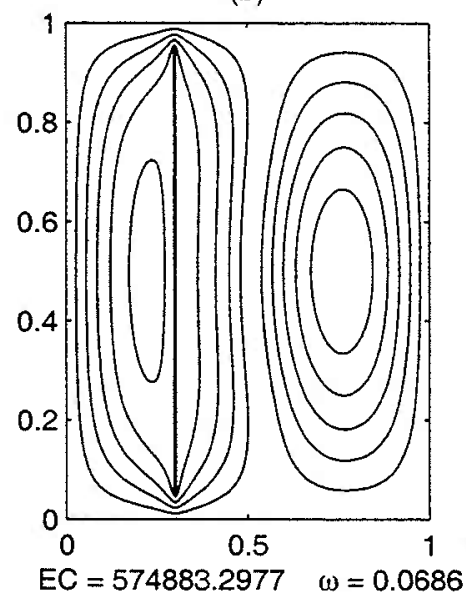

(e)

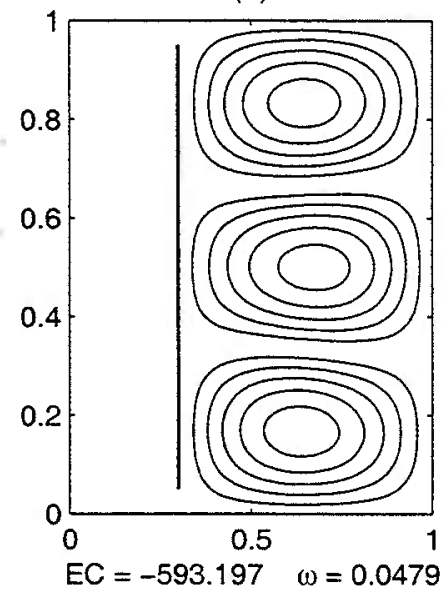

(h)

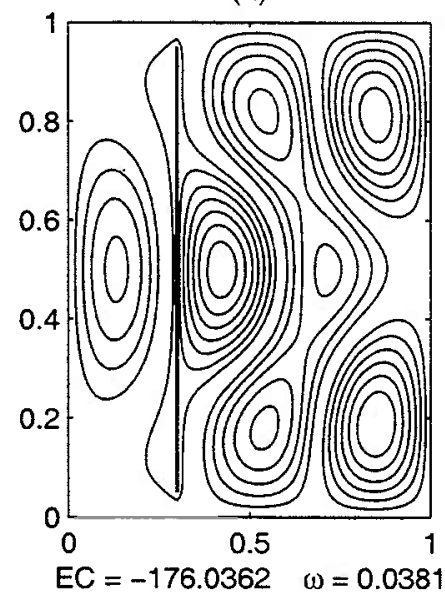

(c)

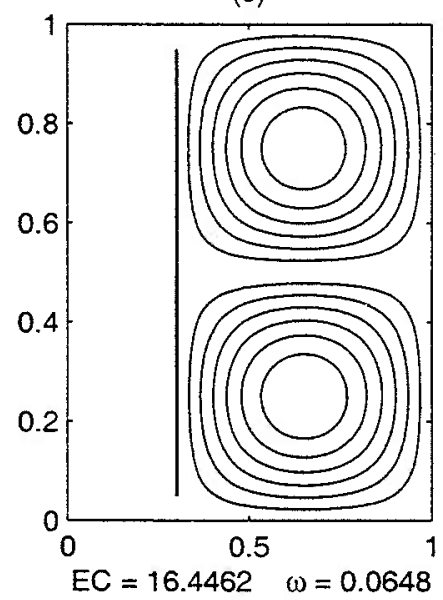

(f)

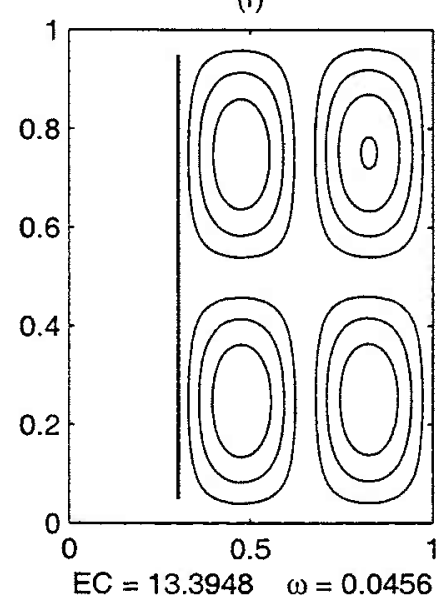

(i)

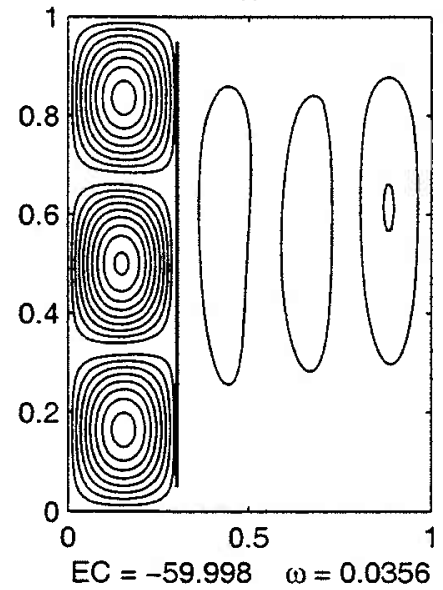

Figure B-6: Contour plots for $x_{I}=0.3, x_{F}=0.7, d=0.05, \gamma=10^{-8}$, and the asymmetric meridional structure of the forcing. The forcing frequencies, under each plot, correspond to the first nine peaks in the full basin variance graph in Figure 5 (a). The value EC shown next to the forcing frequency is the circulation on the east side of the barrier (see appendix $\mathrm{C}$ for calculation of $\mathrm{EC}$ ). 
(a)

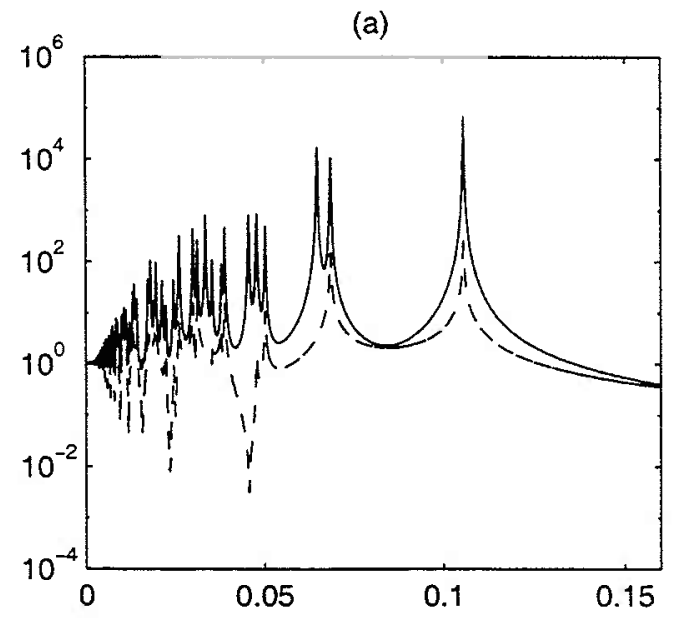

(c)

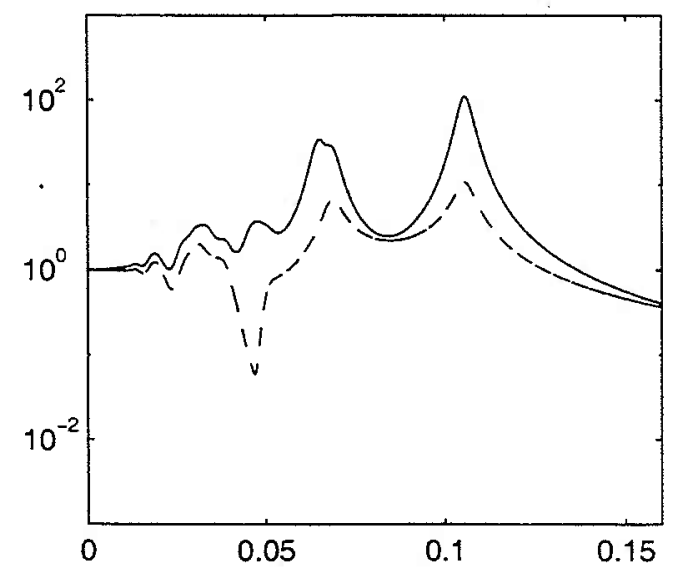

(e)

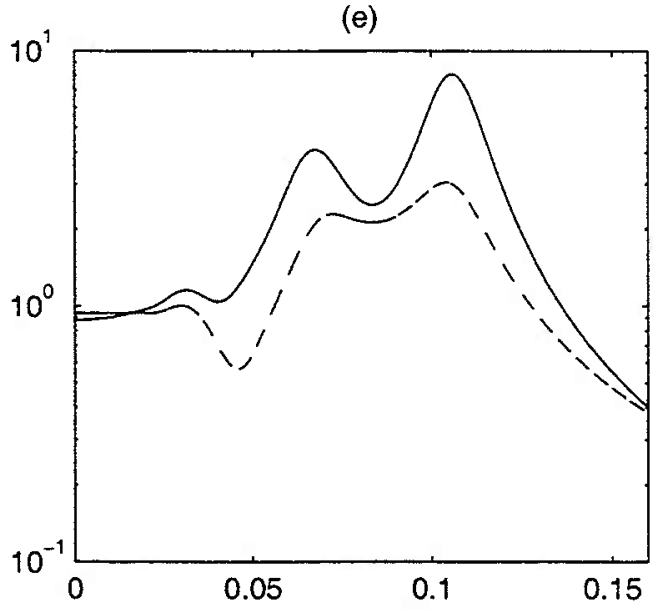

(b)

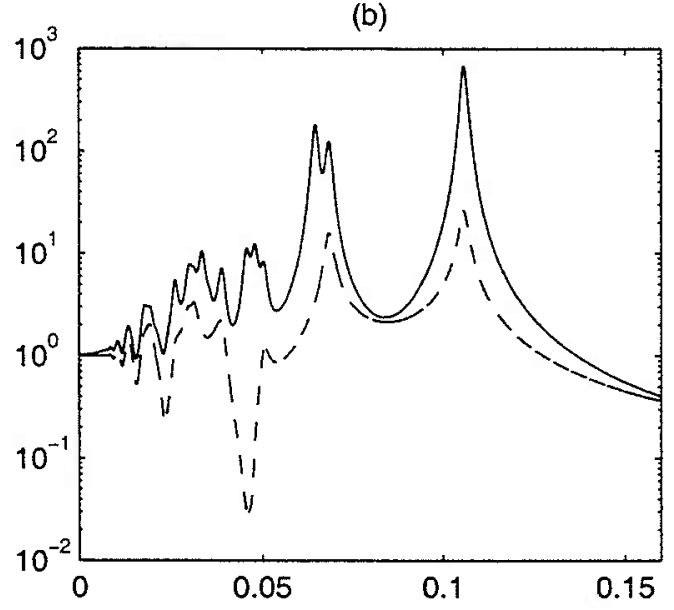

(d)

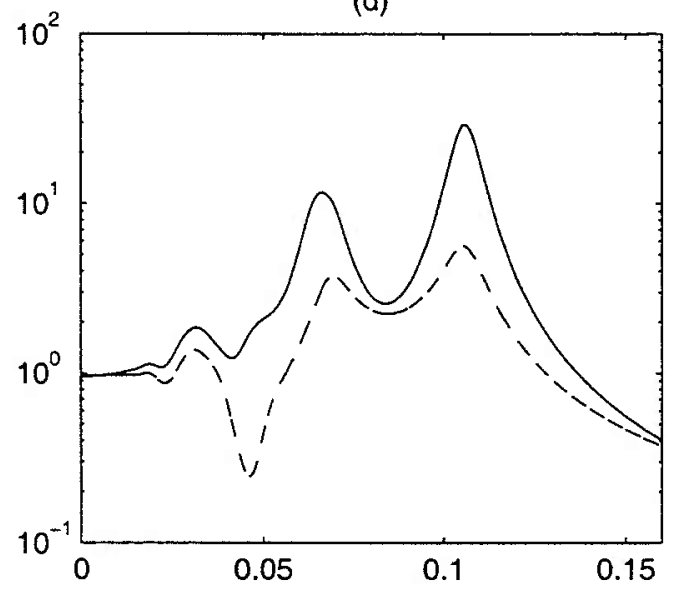

(f)

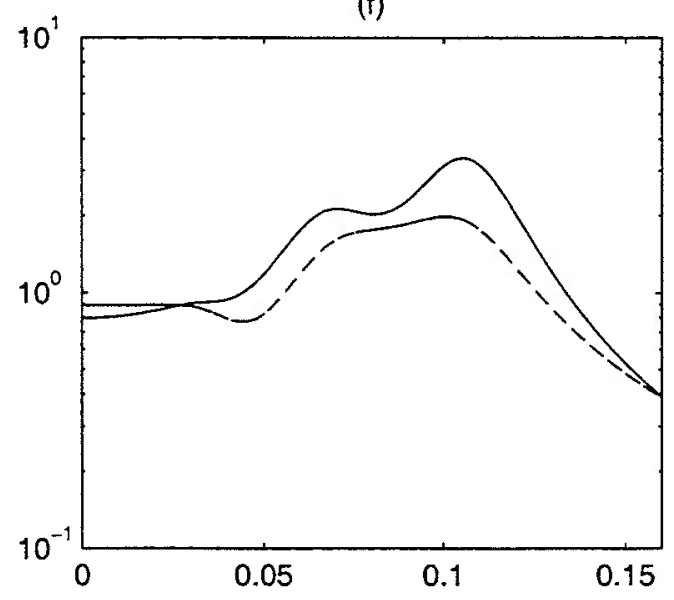

Figure B-7: Semilog plots of $\left|\phi_{I}\right|(--)$ and full basin variance vs forcing frequency $\omega_{F_{n . d}}$ for $x_{I}=0.3, x_{F}=0.7, d=0.05$, and asymmetric meridional structure of the forcing, and varying friction (a) $\gamma=0.0001$, (b) $\gamma=0.001$, (c) $\gamma=0.0025$, (d) $\gamma=0.005$, (e) $\gamma=0.01$, (f) $\gamma=0.0167$. 
(a) Full Basin Variance vs Bottom Friction Parametery

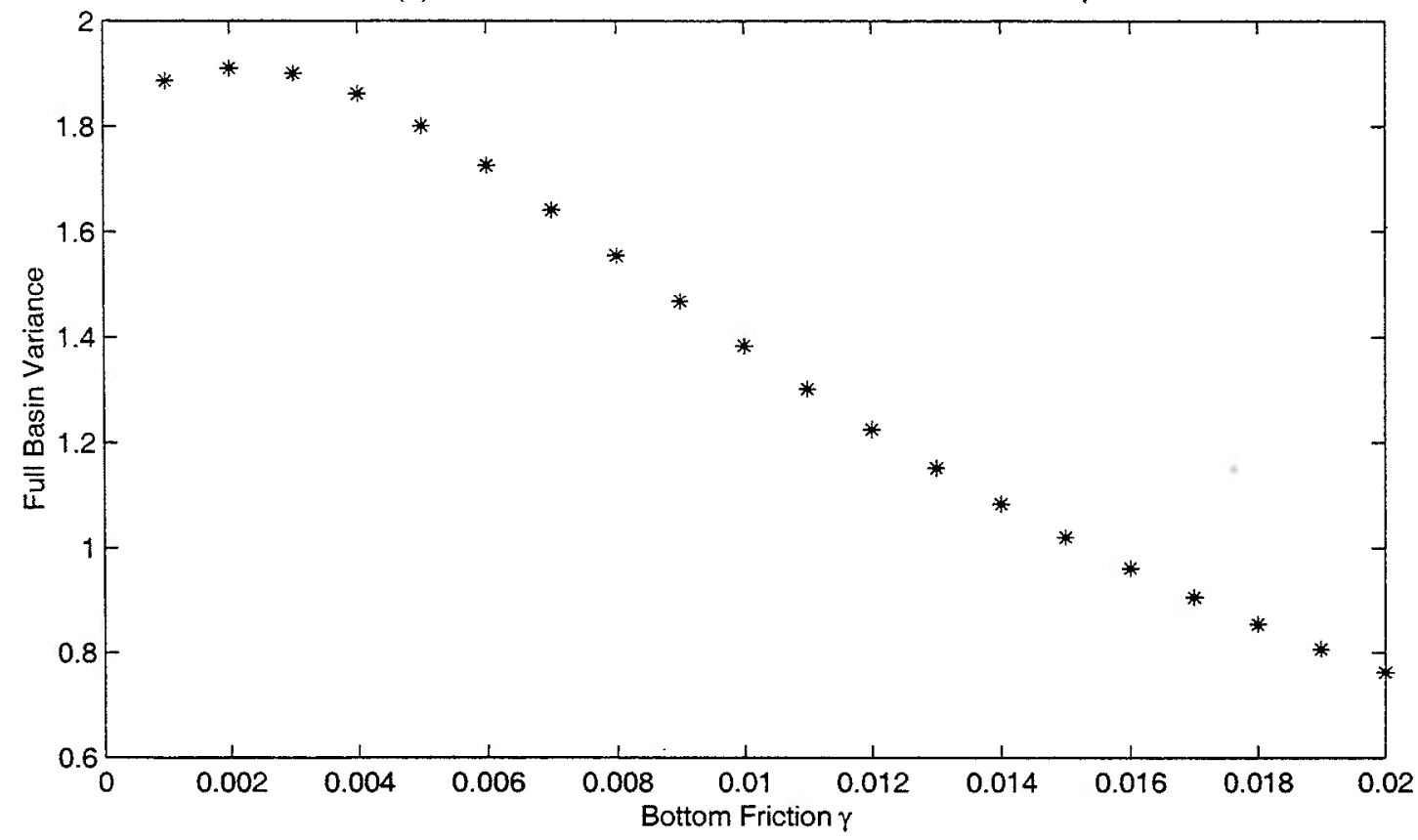

(b) Eastern Sub-Basin Variance vs $\gamma$

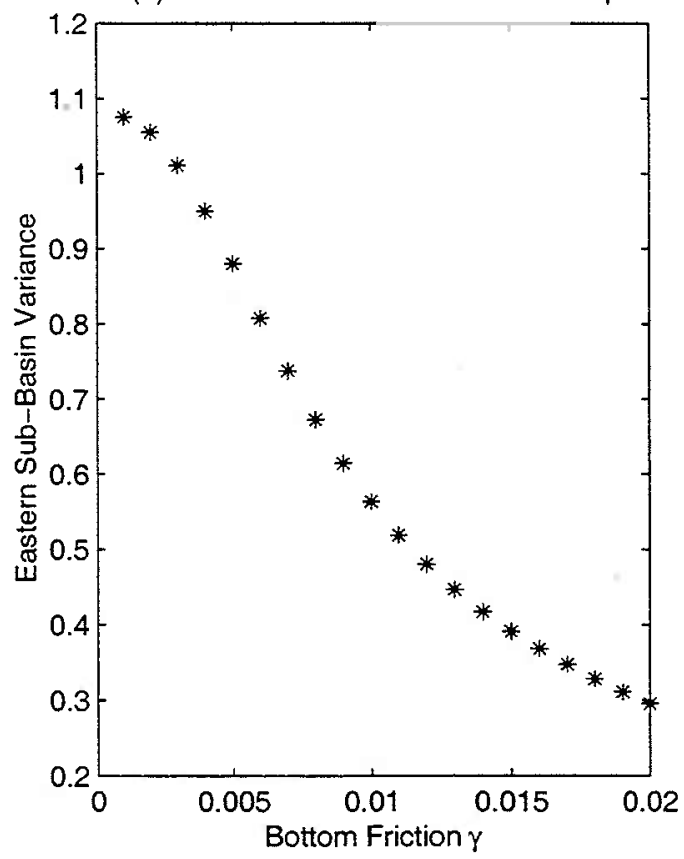

(c) Western Sub-Basin Variance vs $\gamma$

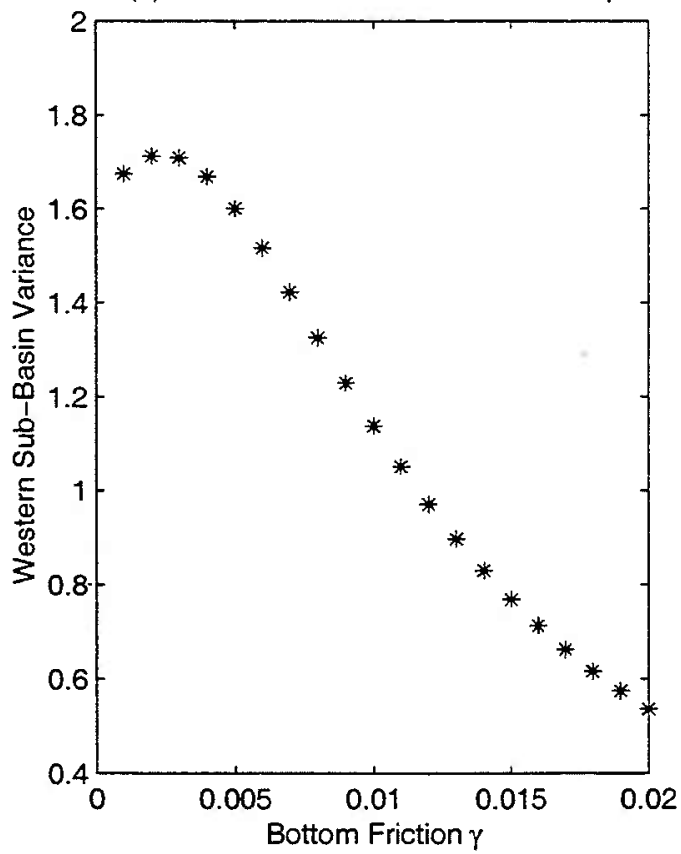

Figure B-8: The (a) full basin, (b) eastern sub-basin and (c) western sub-basin variance response vs the bottom friction parameter $\gamma$ for $\omega_{F n, d,}=0.08$ for the barrier basin analytical solution. 
Envelope Contour Plots as Bottom Friction $(\gamma)$ Increases $\omega_{F}=0.08$

(a)

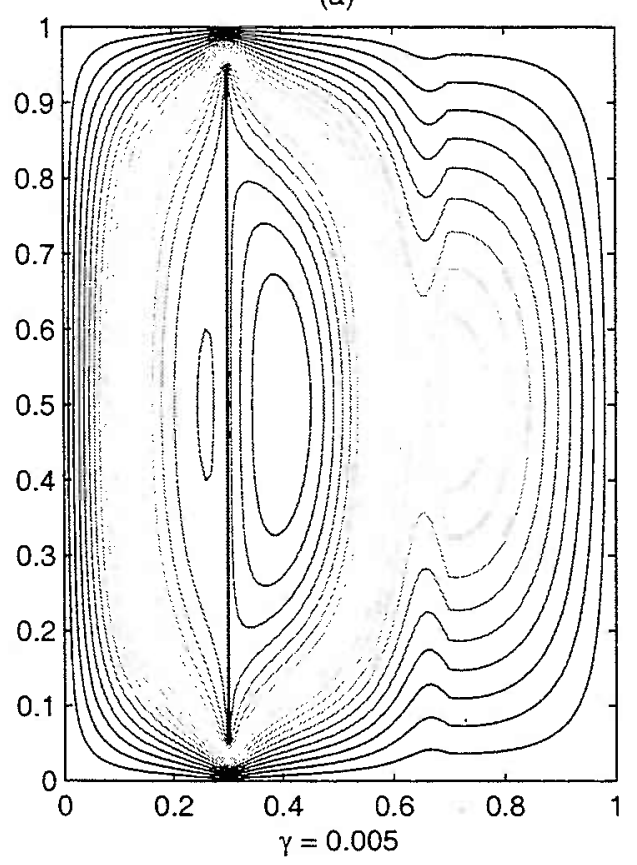

(c)

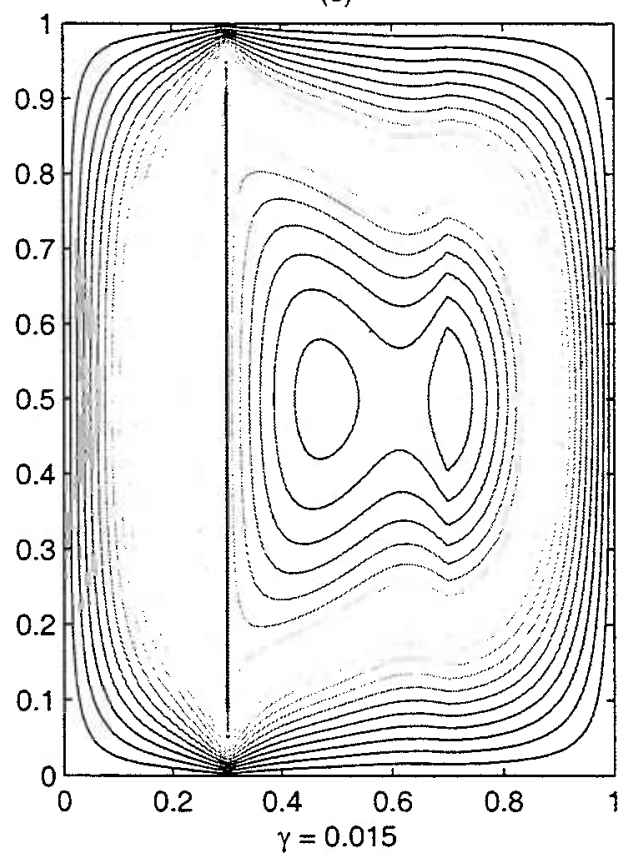

(b)

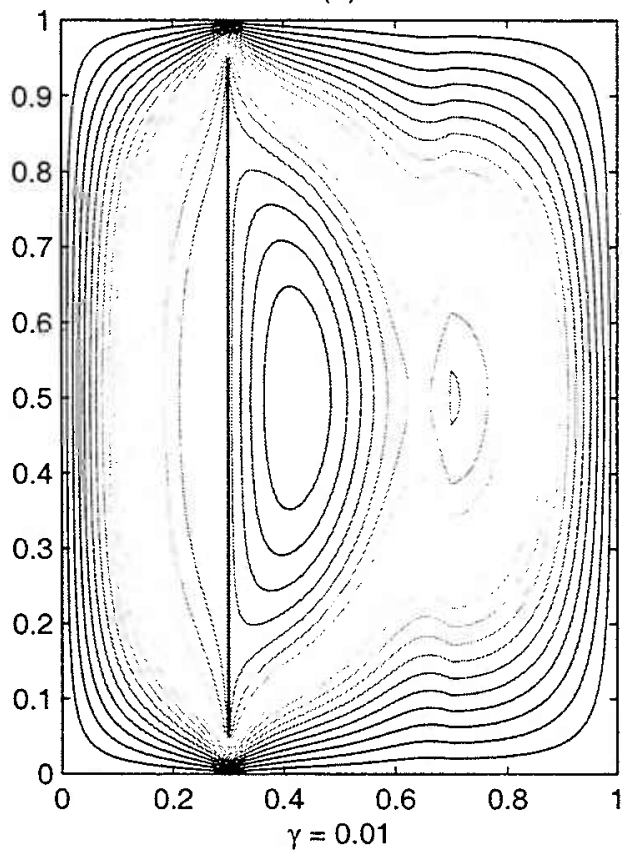

(d)

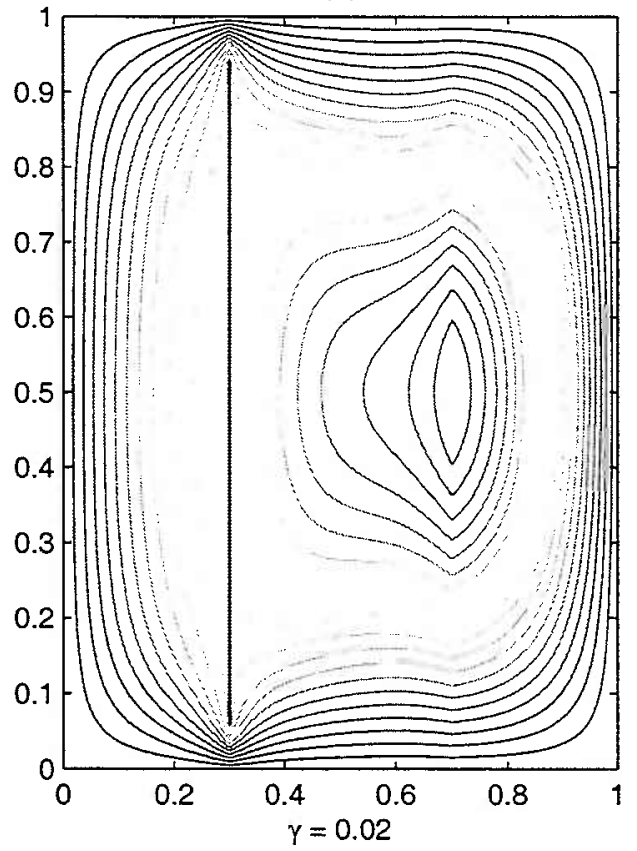

Figure B-9: Contours of the envelope (i.e. absolute value $|\phi(x, y)|$ ) for $\omega_{F_{n . d .}}=0.08$ and certain values of the bottom friction parameter $\gamma$ in the linear barrier basin solution. (a) $\gamma=0.005$, (b) $\gamma=0.01$, (c) $\gamma=0.015$, (d) $\gamma=0.02$. 


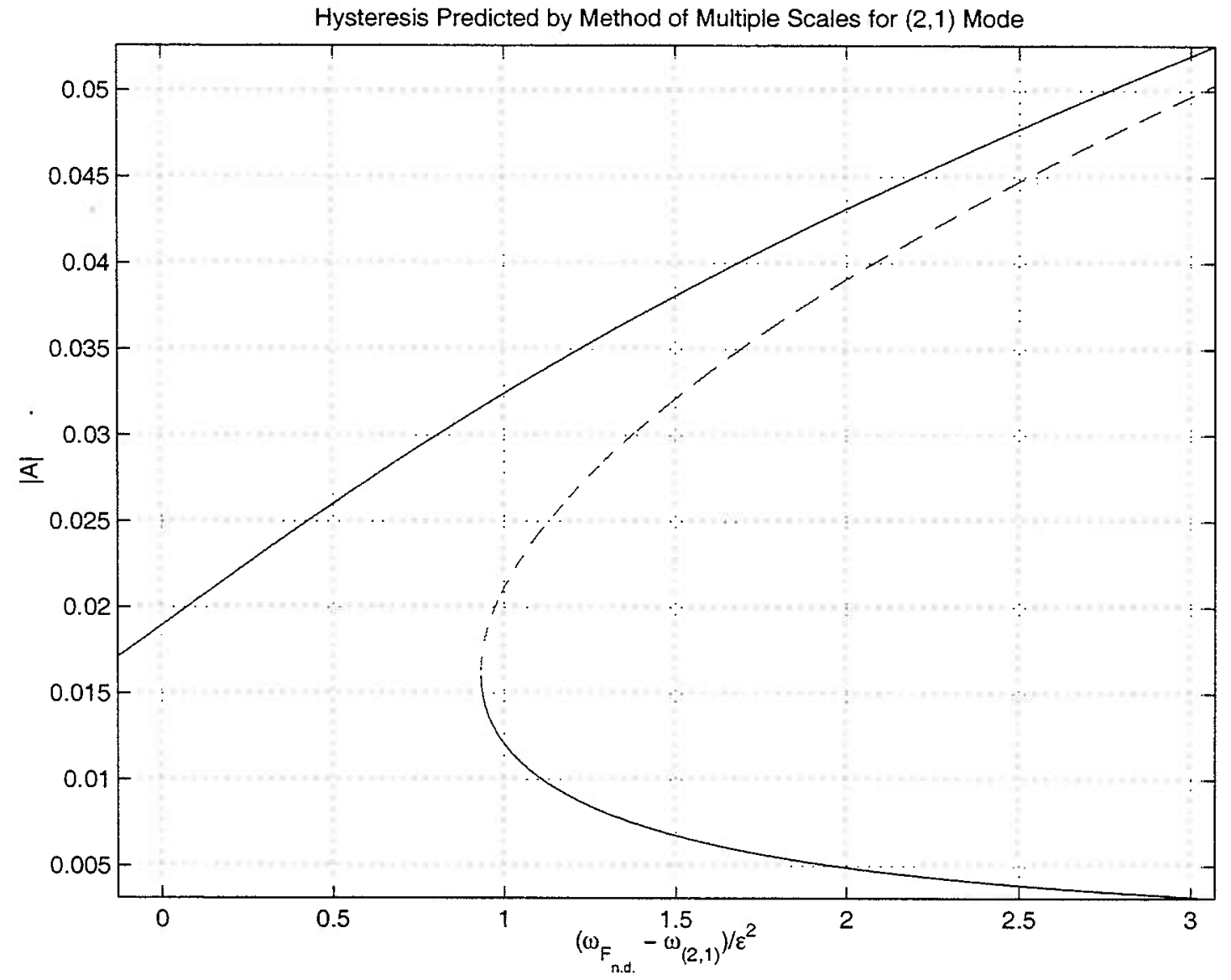

Figure B-10: Amplitude response curve calculated using the method presented in [3] The parameters $k=0, m=2, n=1, f=1$, and, $\delta=0.126$ correspond to our first numerical run where $\left(\frac{\delta_{I}}{L}\right)_{W N}^{2}=0.002$. 


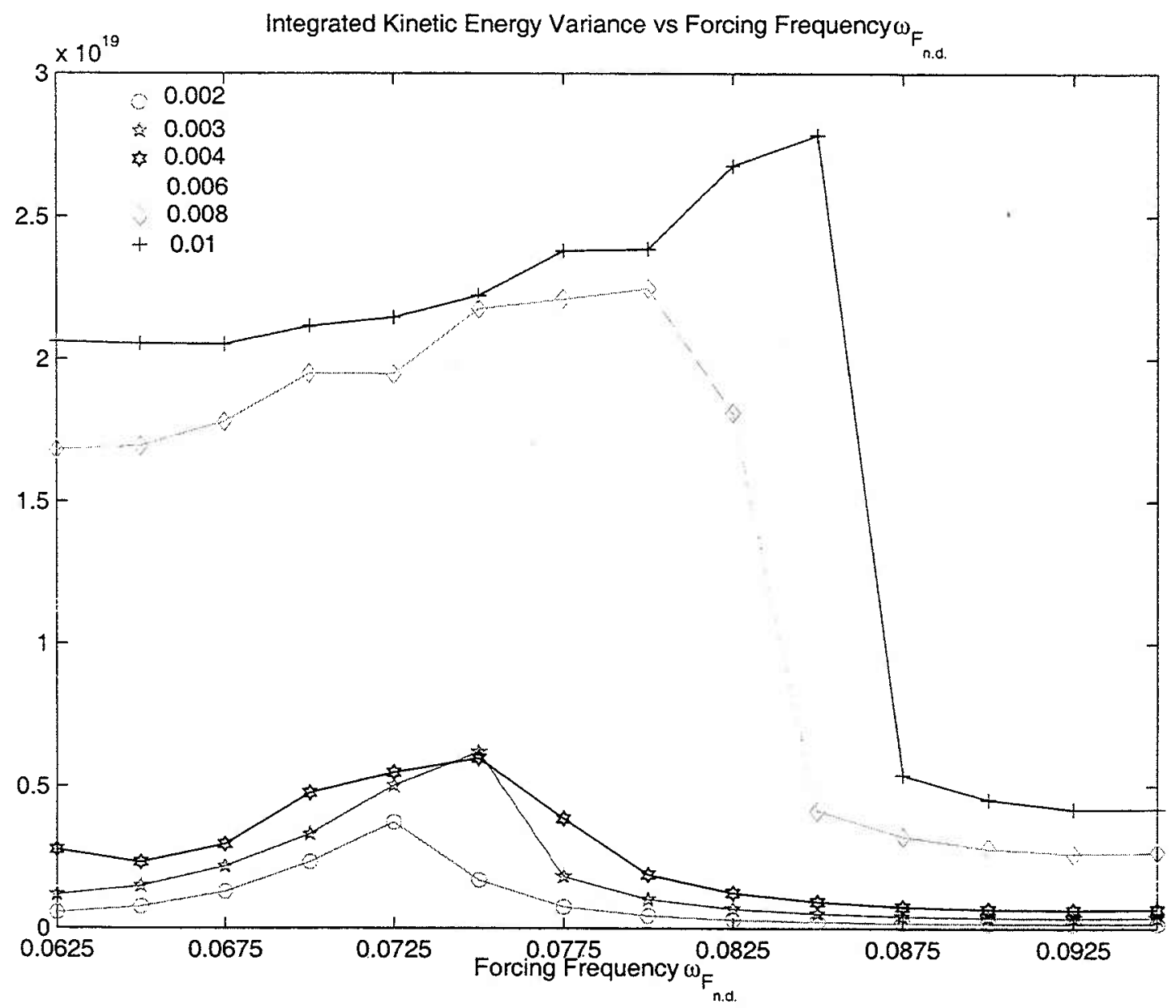

Figure B-11: Mean integrated kinetic energy vs forcing frequency, $\omega_{F_{n . d}}$, for the series of numerical runs with increasing nonlinearity. Notice how the peaks lean towards higher frequencies and then ultimately disintegrate. Also, note the transition in the solution occurring at the nondimensional frequencies $\omega_{F_{n . d} .}, 0.0775,0.08,0.0875$, and 0.085 for the wind forcing strengths $\left(\frac{\delta_{I}}{L}\right)_{W N}^{2}=0.004,0.006,0.008$, and 0.01 , respectively. 
(a) $\left(\delta_{1} / L\right)_{W N}^{2}=0.004$

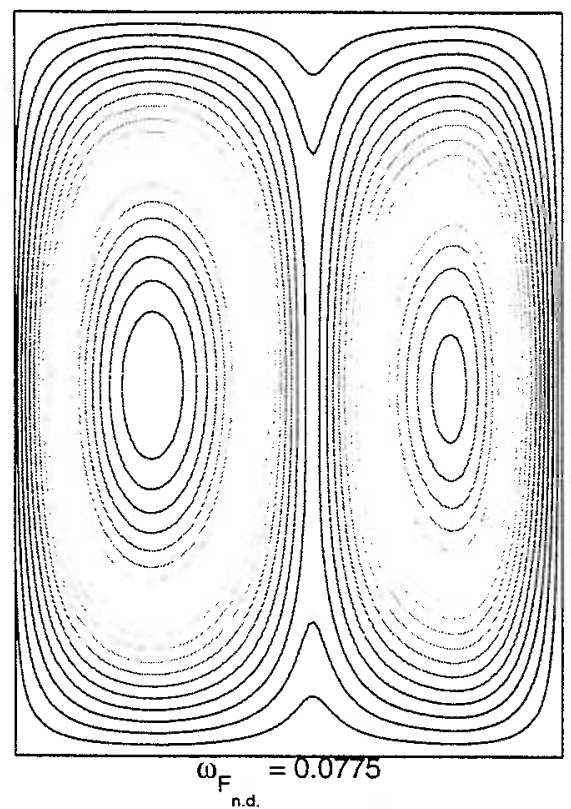

(c) $(\delta / L)_{W N}^{2}=0.008$

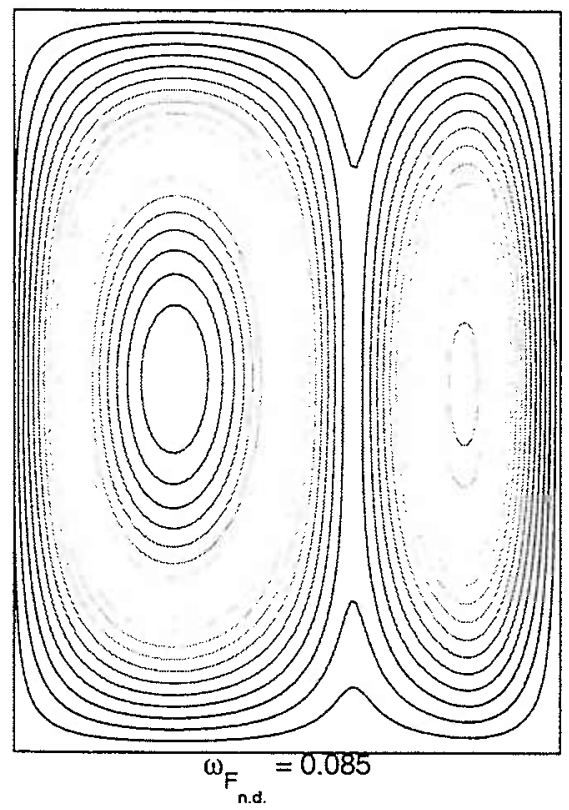

(b) $\left(\delta_{1} / \mathrm{L}\right)_{W N}^{2}=0.006$

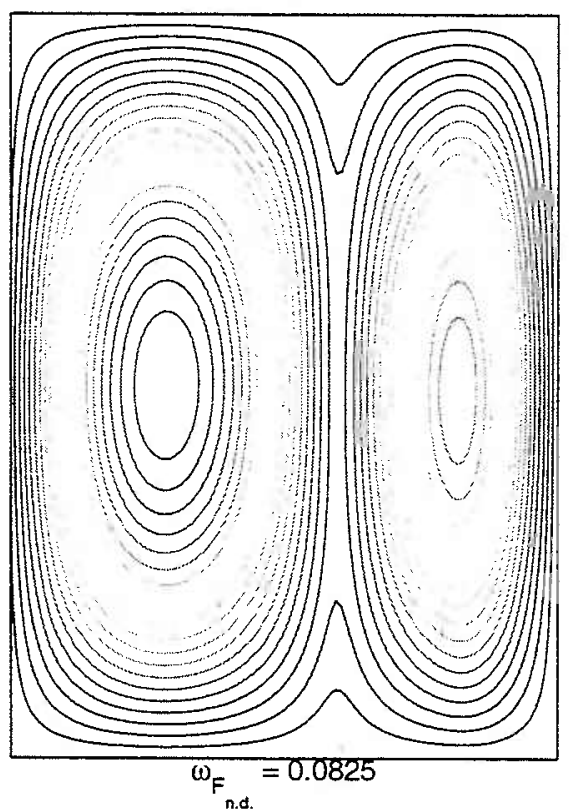

(d) $(\delta / L)_{W N}^{2}=0.01$

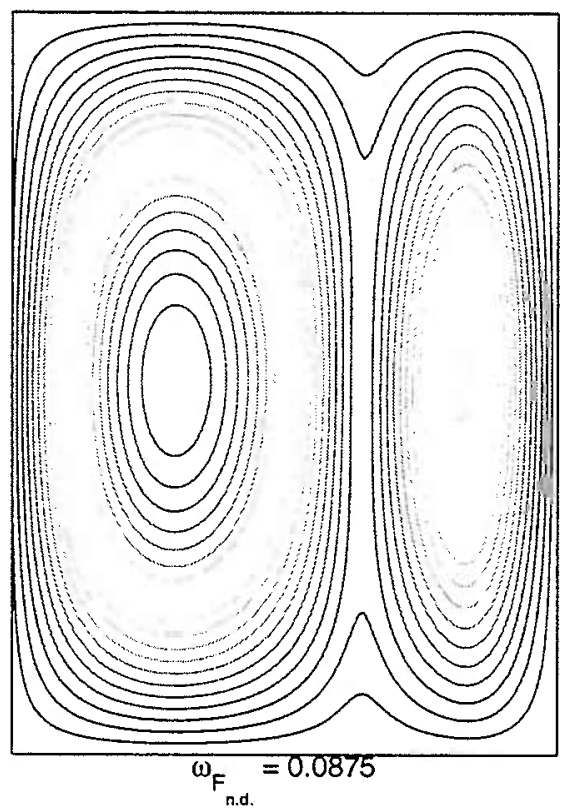

Figure B-12: Envelopes calculated over one forcing period demonstrating that the weakly nonlinear solution is dominated by the $(2,1)$ mode just before the transition which occurs when the forcing frequency is decreased. (a) $\left(\frac{\delta_{I}}{L}\right)_{W N}^{2}=0.004$ and $\omega_{F_{n . d .}}=0.0775$, (b) $\left(\frac{\delta_{I}}{L}\right)_{W N}^{2}=0.006$ and $\omega_{F_{n . d .}}=0.0825$, (c) $\left(\frac{\delta_{L}}{L}\right)_{W N}^{2}=0.008$ and $\omega_{F_{n . d .}}=0.085$, and (d) $\left(\frac{\delta_{I}}{L}\right)_{W N}^{2}=0.01$ and $\omega_{F_{n . d .}}=0.0875$. 
(a) $\left(\delta_{\mathrm{I}} / L\right)_{W N}^{2}=0.004$

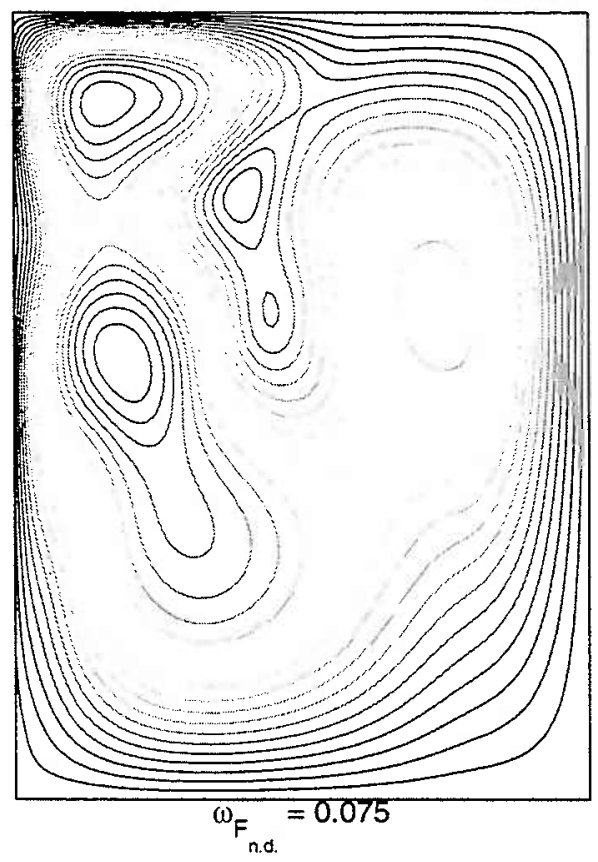

(c) $\left(\delta_{\mathrm{l}} / \mathrm{L}\right)_{\mathrm{WN}}^{2}=0.008$

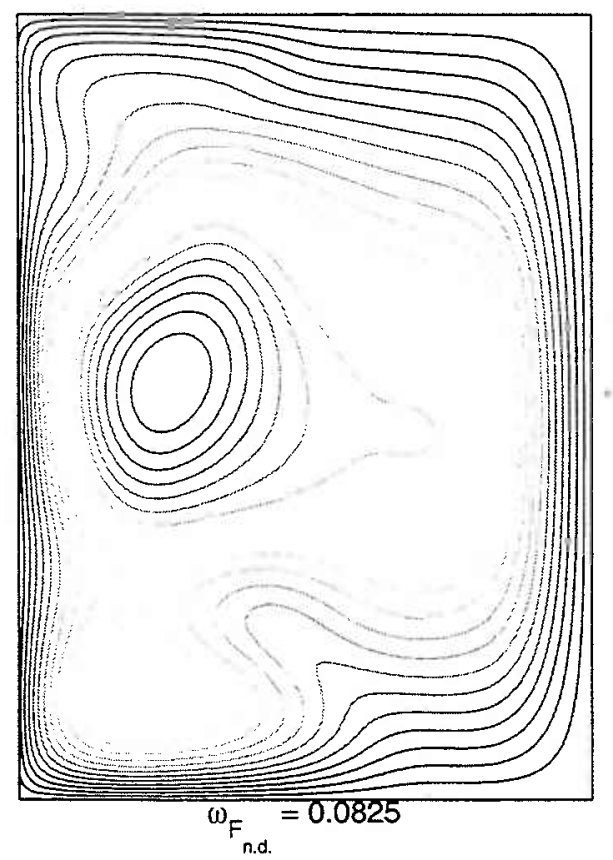

(b) $\left(\delta_{1} / L\right)_{W N}^{2}=0.006$

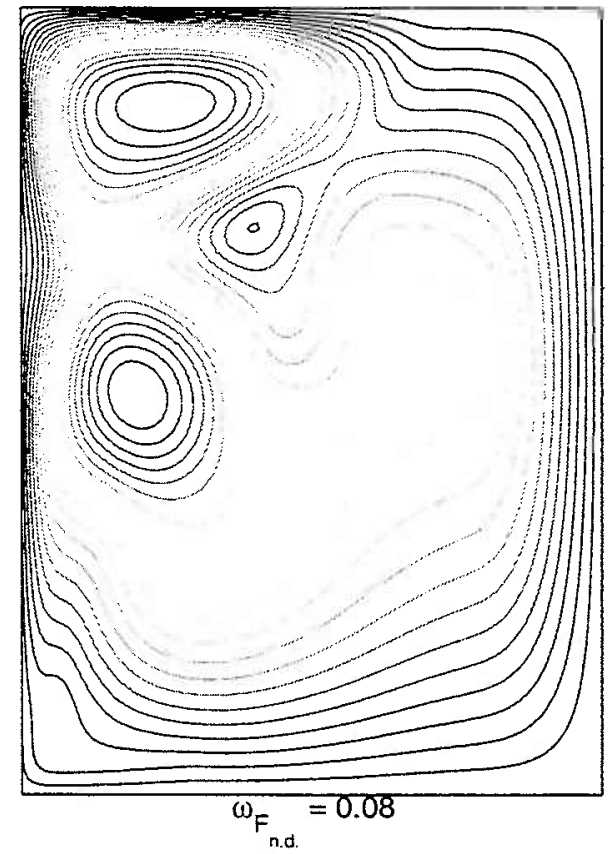

(d) $\left(\delta_{\mathrm{I}} / \mathrm{L}\right)_{\mathrm{WN}}^{2}=0.01$

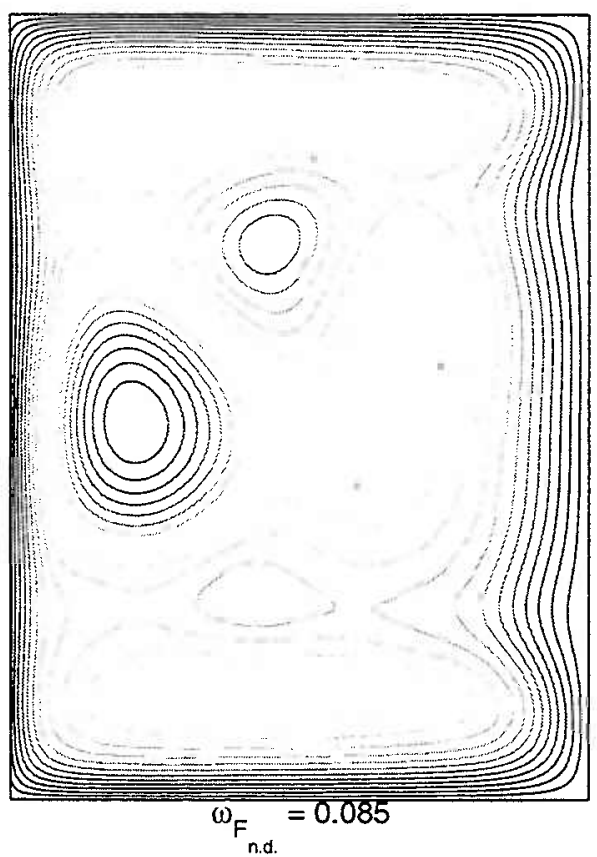

Figure B-13: Envelope plots taken over one forcing period showing the solution just after the transition to the very nonlinear solution when the forcing frequency is decreased.(a) $\left(\frac{\delta_{I}}{L}\right)_{W N}^{2}=0.004$ and $\omega_{F_{n . d .}}=0.0775$, (b) $\left(\frac{\delta_{I}}{L}\right)_{W N}^{2}=0.006$ and $\omega_{F_{n . d .}}=0.0825$, (c) $\left(\frac{\delta_{I}}{L}\right)_{W N}^{2}=0.008$ and $\omega_{F_{n . d .}}=0.085$, and (d) $\left(\frac{\delta_{I}}{L}\right)_{W N}^{2}=0.01$ and $\omega_{F_{n . d .}}=0.0875$ 
Contours of $\Psi$ at 2 day Intervals for $N L=0.004 \omega_{F}=0.0725$

(a)

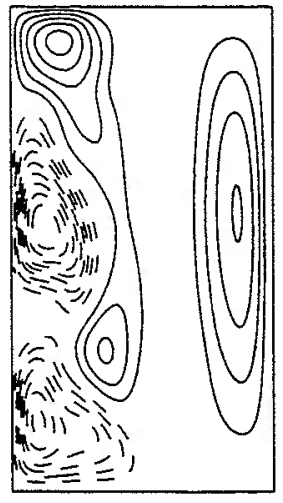

(e)
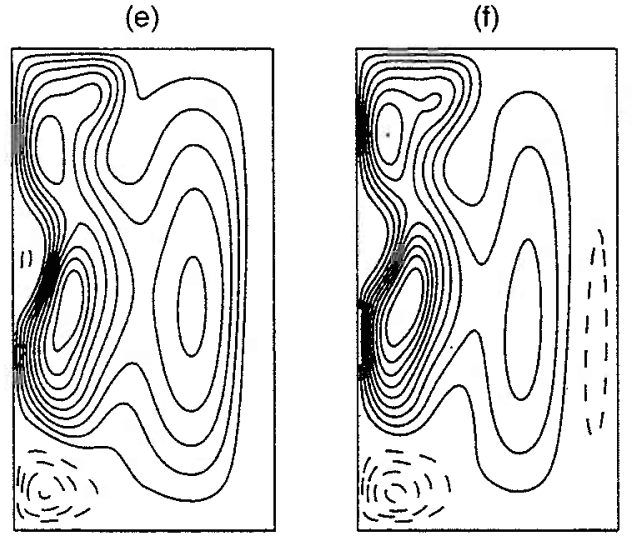

(i)

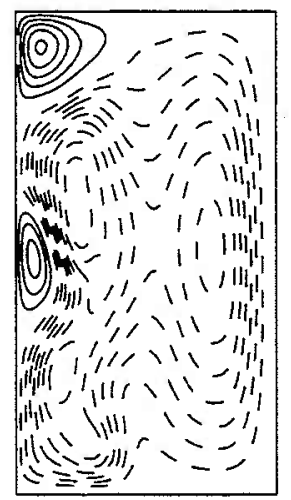

(c)

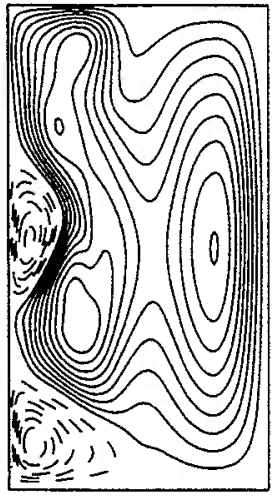

(g)

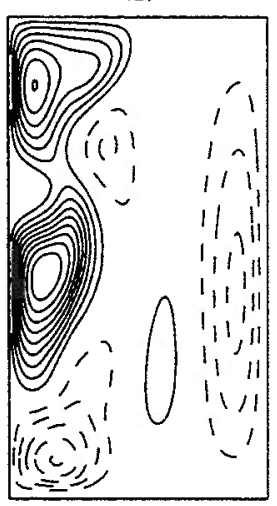

(k)

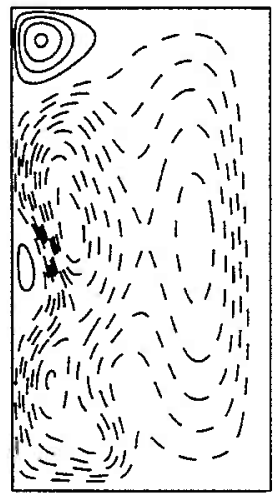

(d)

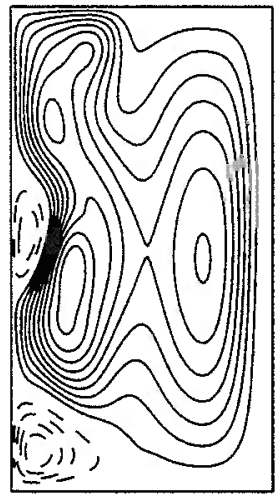

(h)

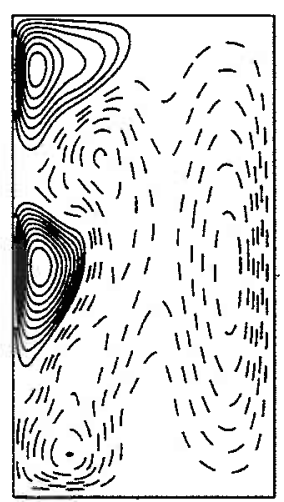

Figure B-14: Contours of the stream function, $\Psi$, at 2 day intervals for the solution $\left(\frac{\delta_{I}}{L}\right)_{W N}^{2}=0.004$ and $\omega_{F n . d}=0.0725$. The contours span 24 days of the 25.08 day forcing period. Solid lines correspond to positive stream lines and dotted lines correspond to negative streamlines. The contours document the westward propagation of the solution during one forcing period. Note $N L$ in the figure denotes $\left(\frac{\delta_{I}}{L}\right)_{W N}^{2}$. 
(a)

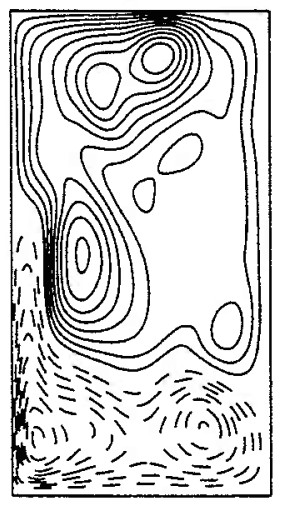

(e)

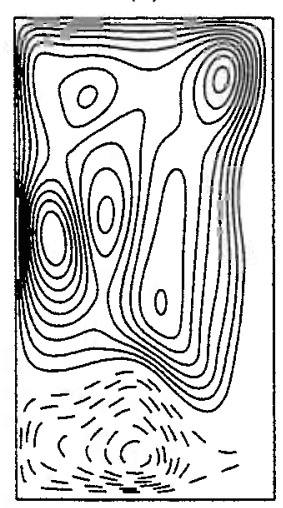

(i)

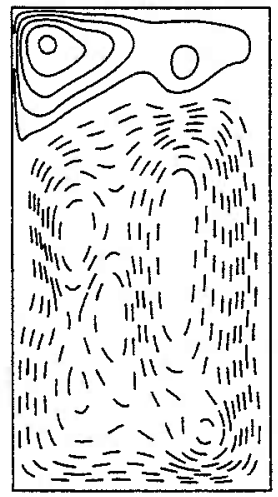

(b)

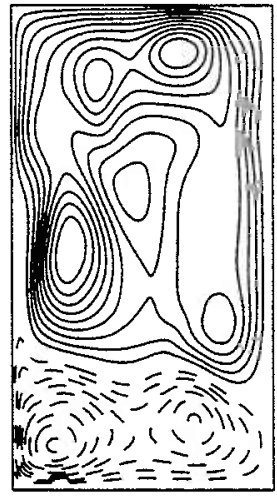

(f)

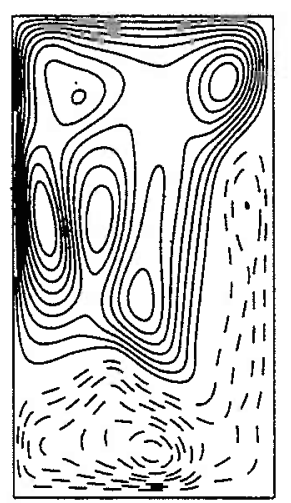

(j)

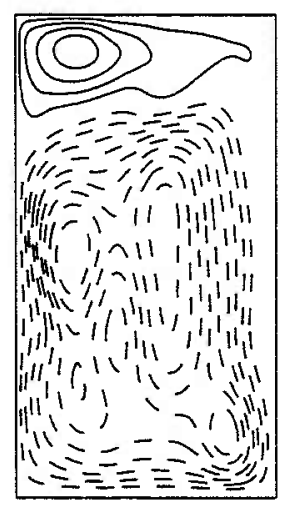

(c)

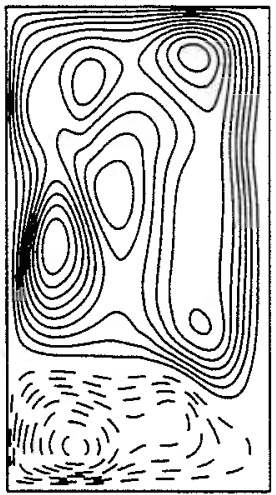

(g)

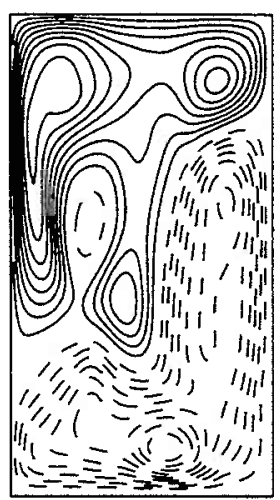

(k)

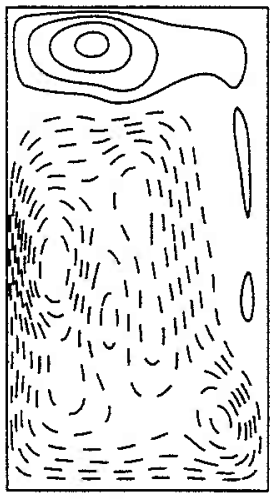

(d)

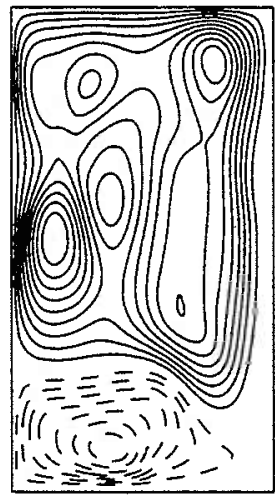

(h)

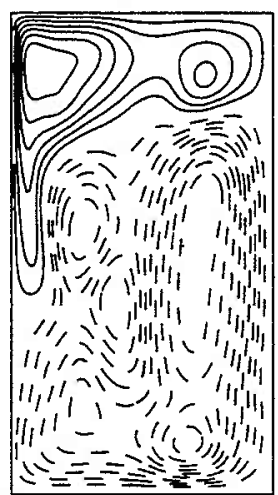

(I)

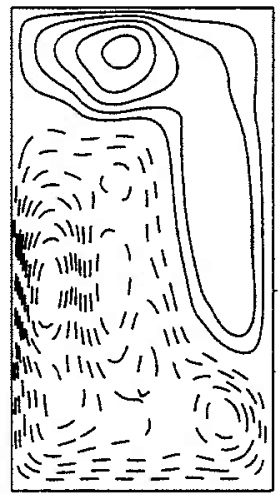

Figure B-15: Contours of the stream function, $\Psi$, at 2 day intervals for the solution $\left(\frac{\delta_{L}}{L}\right)_{W N}^{2}=0.01$ and $\omega_{F n . d .}=0.0725$. The contours span 24 days of the 25.08 day forcing period. Solid lines correspond to positive stream lines and dotted lines correspond to negative streamlines. The contours document the westward propagation of the solution during one forcing period. Note $N L$ in the figure denotes $\left(\frac{\delta_{I}}{L}\right)_{W N}^{2}$. 
$\mathrm{U}(-$.$) , and \mathrm{V}(--)$ in $[\mathrm{cm} / \mathrm{s}]$
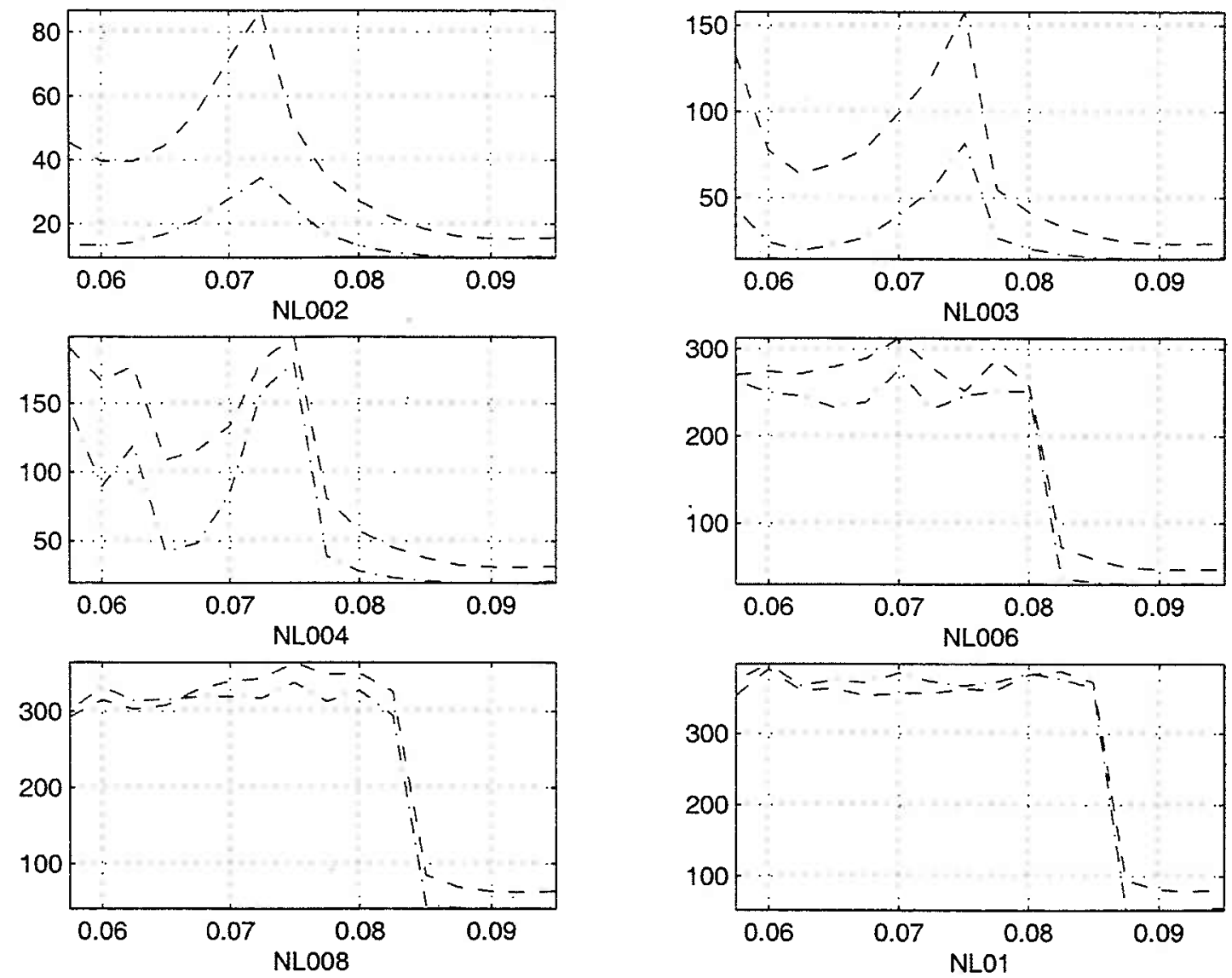

Figure B-16: The maximum zonal and meridional velocities plotted vs the nondimensional forcing frequencies, $\omega_{F_{n . d .}}$, for each set of nonlinear runs. Note $N L$ in the figure denotes $\left(\frac{\delta_{r}}{L}\right)_{W N}^{2}$. 
Fourier Transform of Zonal Velocity at $(800 \mathrm{~km}, 800 \mathrm{~km}) \omega_{\mathrm{F}}=0.0725$

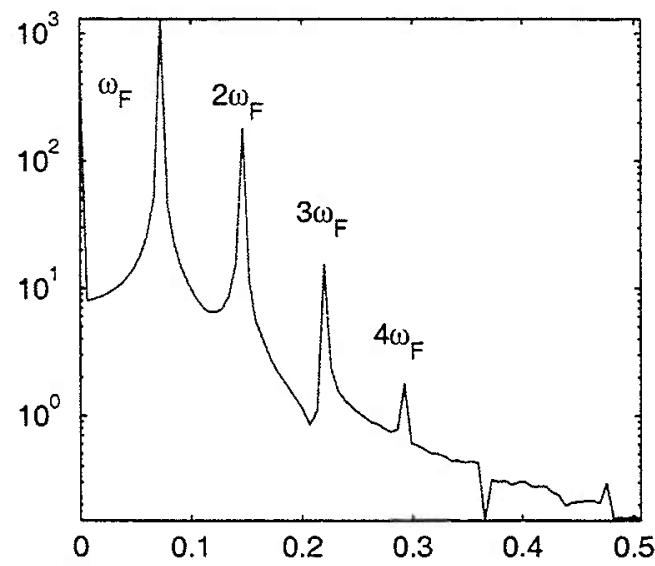

(a) NL0.002

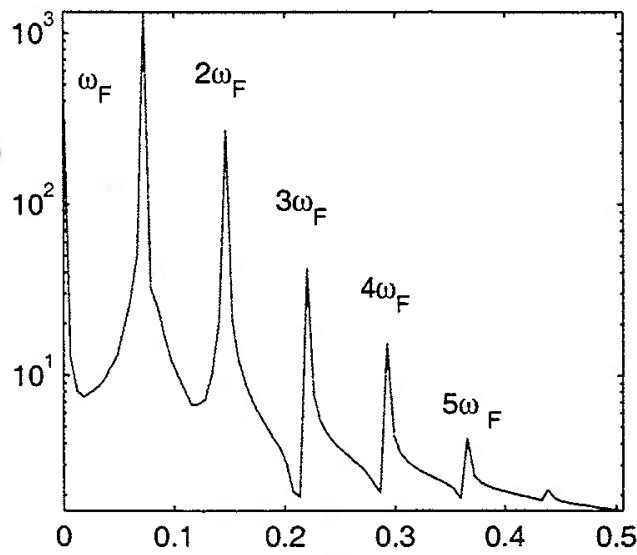

(b) NLO.003

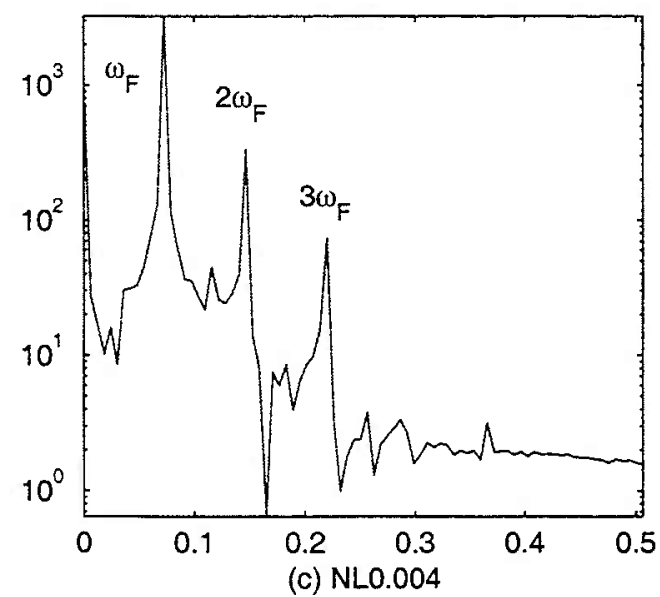

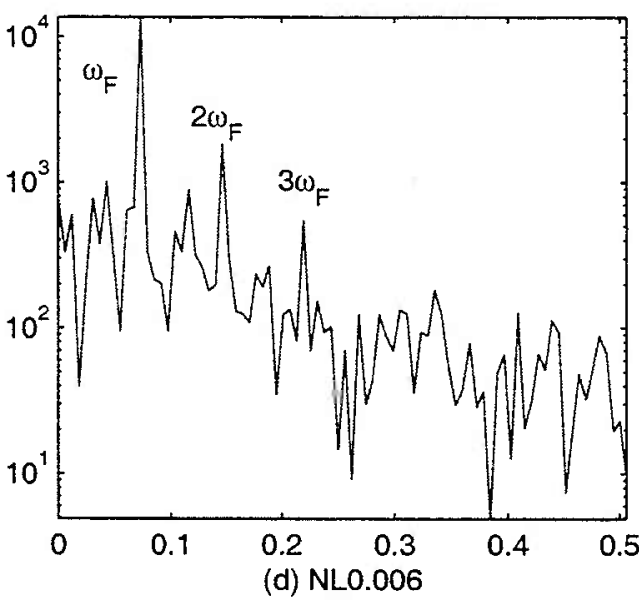

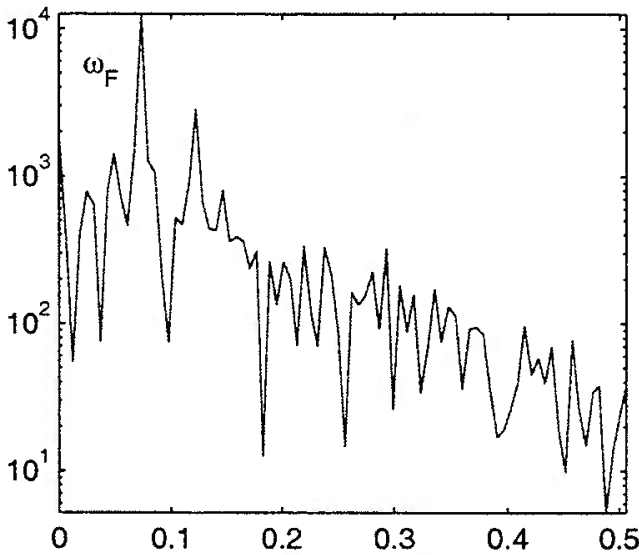

(e) NLO.008

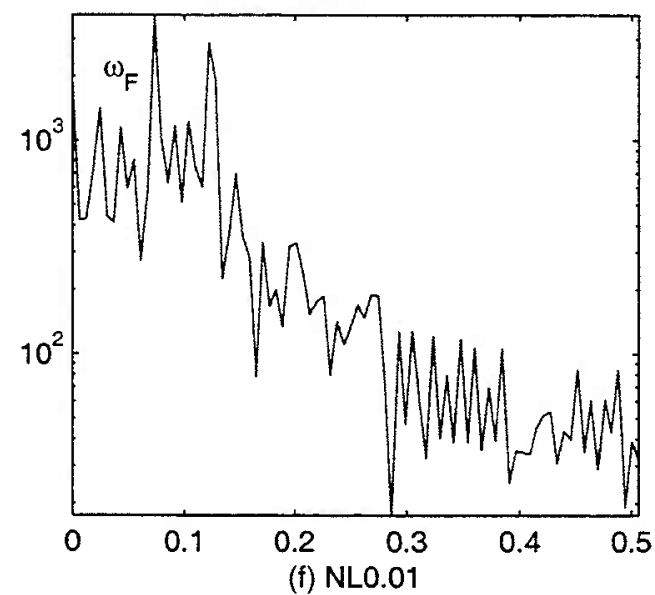

Figure B-17: Fourier transform of the zonal velocity at the point $(800 \mathrm{~km}, 800 \mathrm{~km})$ for the nonlinear solutions with a forcing frequency of $\omega_{F n . d .}=0.0725$. Note $N L$ in the figure denotes $\left(\frac{\delta_{I}}{L}\right)_{W N}^{2}$. 

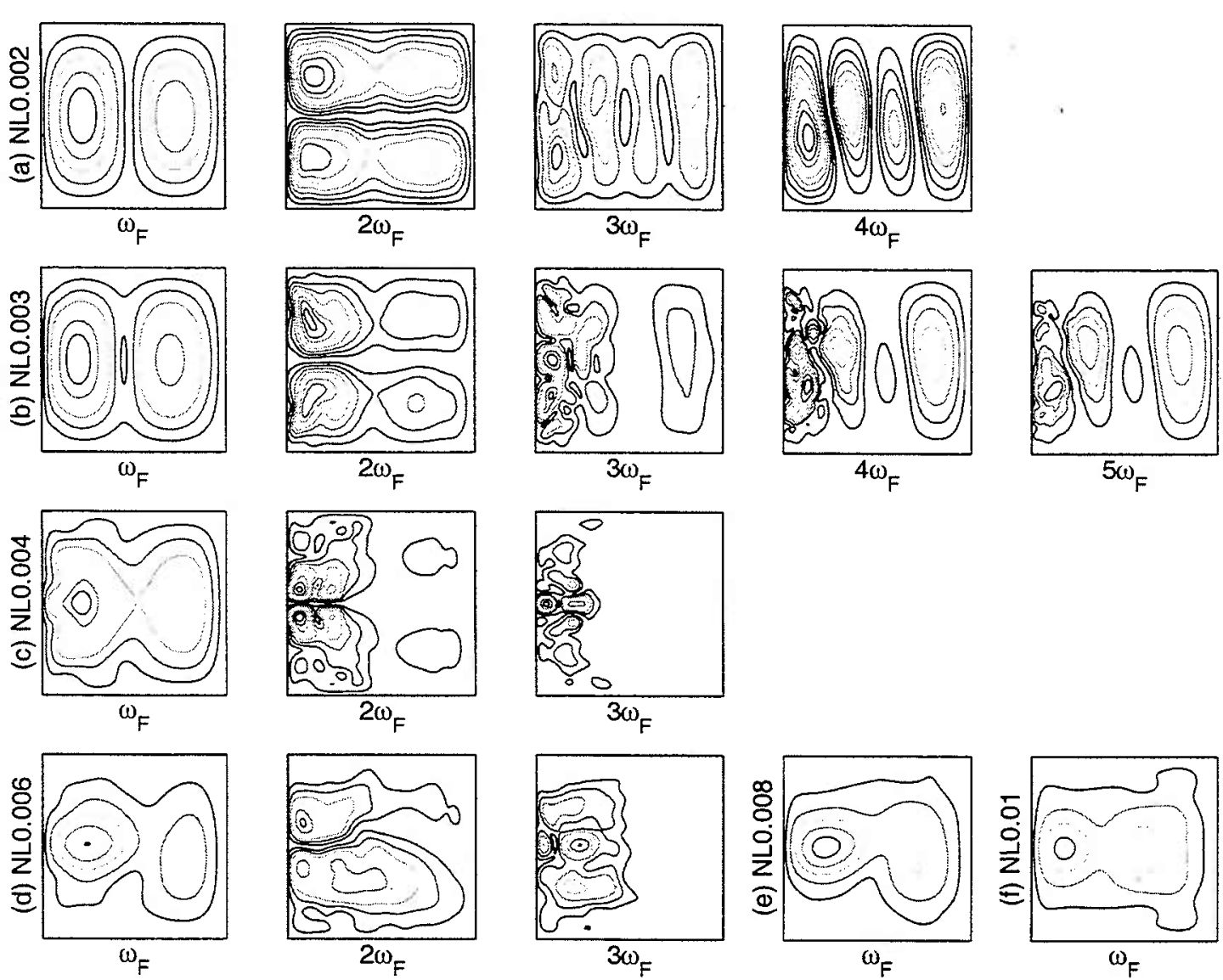

Figure B-18: Contour plots of the absolute values of the spatial coefficients of the terms in a Fourier time series which oscillate at frequencies that are integral multiples of the forcing frequencies. Note $N L$ in the figure denotes $\left(\frac{\delta_{I}}{L}\right)_{W N}^{2}$. 
FFT of Zonal Velocity Over 1200 Days

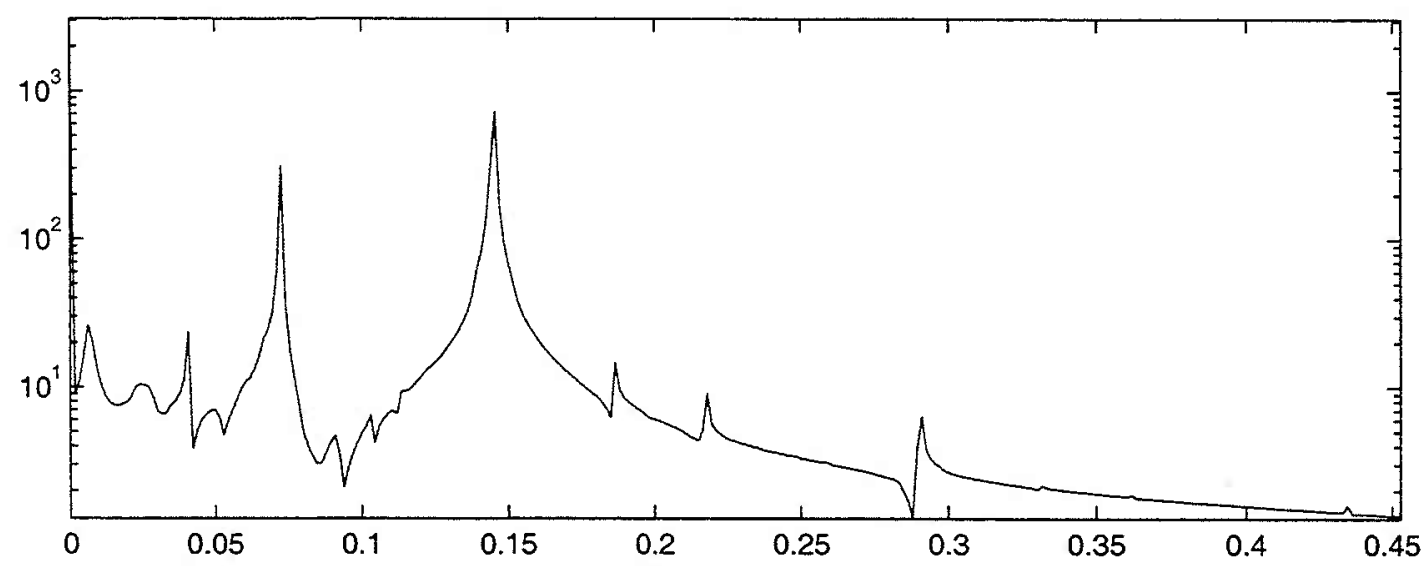

(a) - Zonal Velocity $1000 \mathrm{~km}$ North and $1800 \mathrm{~km}$ East
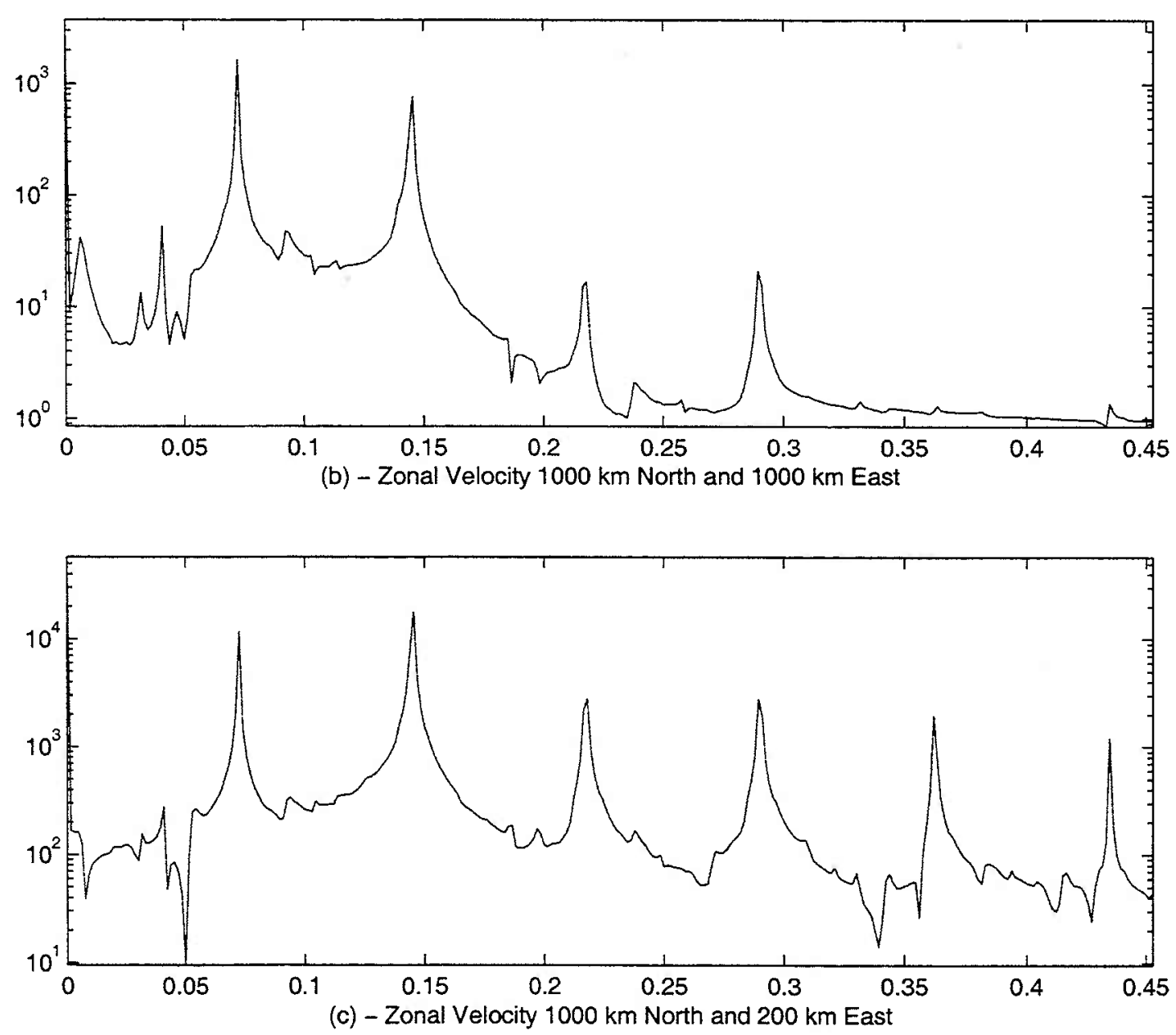

Figure B-19: Fourier transform of the zonal velocity for different points in the basin at mid-latitude over 1200 days (a) east side, (b) middle, (c) west side. $\left(\frac{\delta_{I}}{L}\right)_{W N}^{2}=0.004$ and $\omega_{F n . d .}=0.0725$, note higher frequencies appearing as we proceed west. 
$N L=0.008$ and $\omega_{F}=0.085$
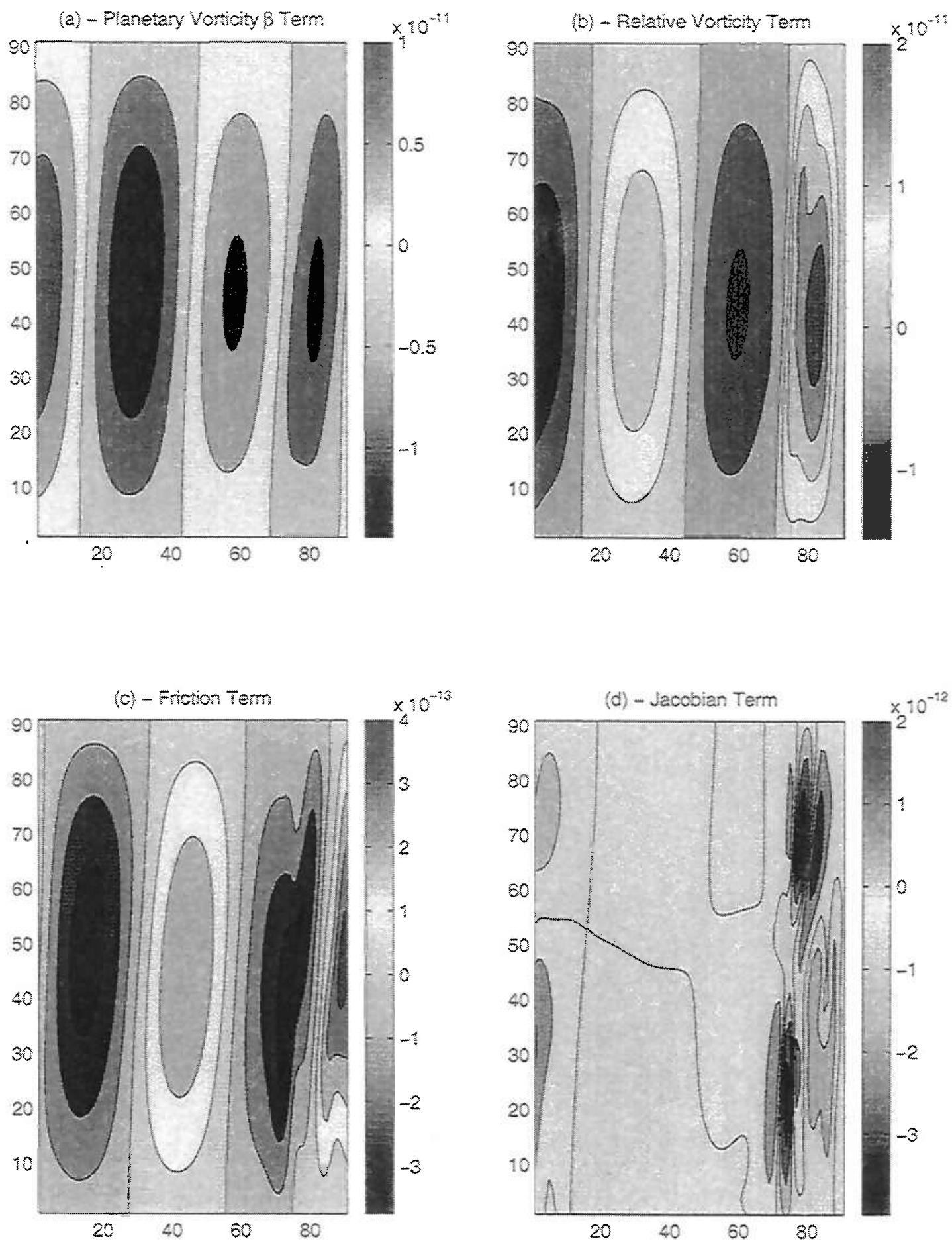

Figure B-20: Finite difference calculation of the model output for the terms in the QG equation excluding the forcing term. $\left(\frac{\delta_{L}}{L}\right)_{W N}^{2}=0.008$ and $\omega_{F n . d .}=0.085$. This is just before the solution transitions to the turbulent regime at $\omega_{F n . d .}=0.0825$. Note $N L$ in the figure denotes $\left(\frac{\delta_{I}}{L}\right)_{W N}^{2}$. 
$\mathrm{NL}=0.008$ and $\omega_{F}=0.085$

(a) - Planetany Voricity 1 Tem

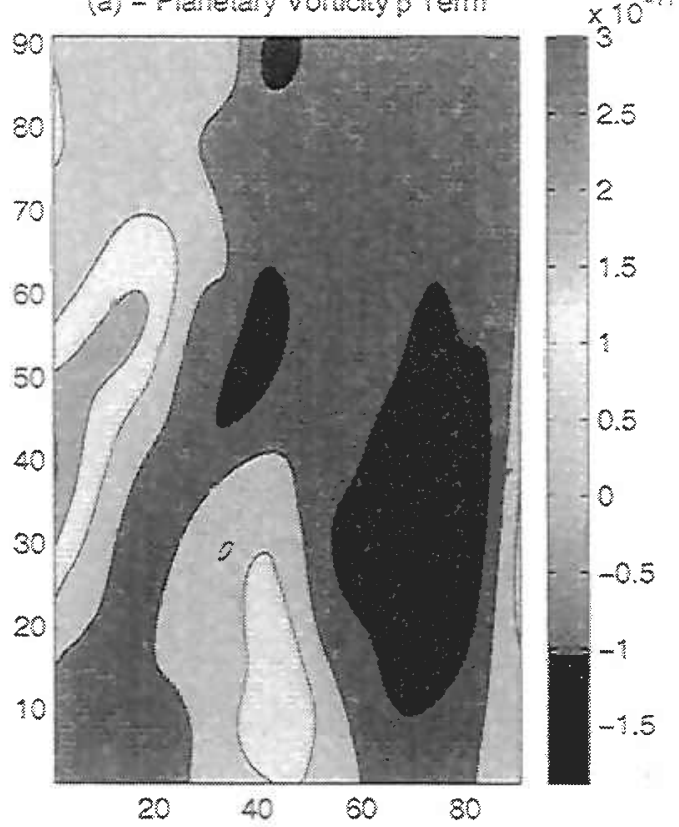

(c) - Fiction Term

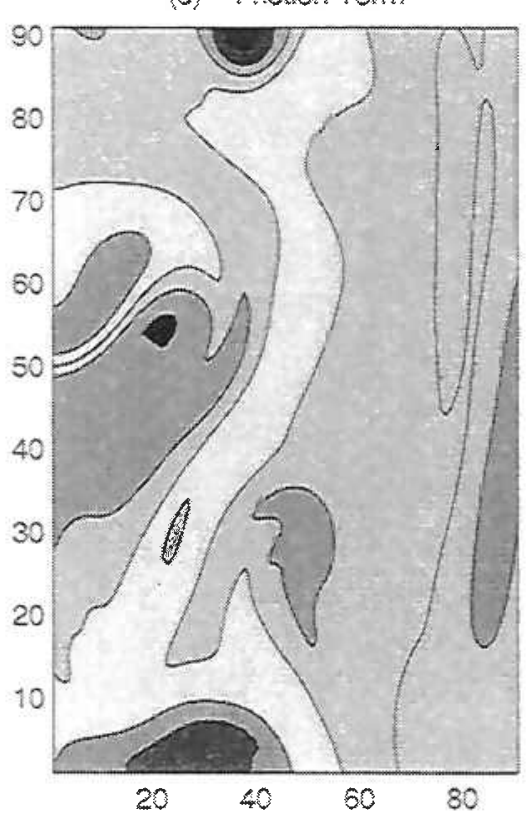

(b) - Reletive Vorticty Term

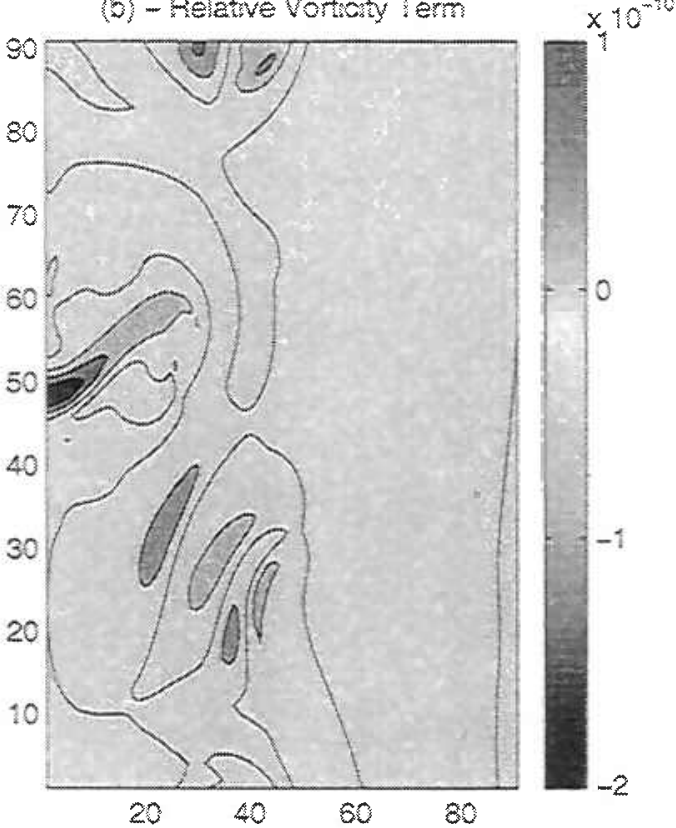

(d) - Jacobian Term

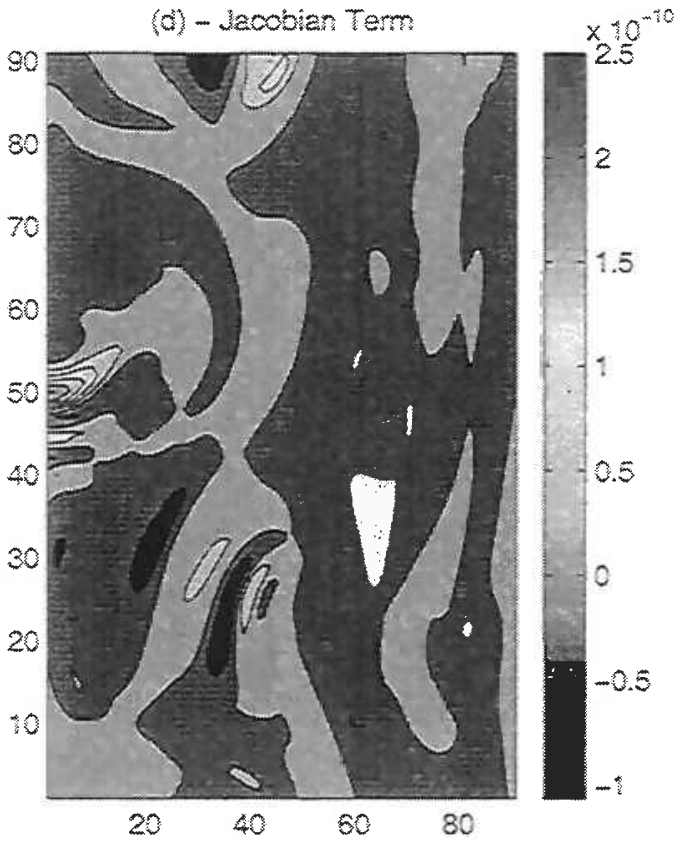

Figure B-21: Finite difference calculation of the model output for the terms in the QG equation excluding the forcing term. The solution has a wind forcing strength $\left(\frac{\delta_{r}}{L}\right)_{W N}^{2}=0.008$ and a forcing frequency $\omega_{F n \text {.d. }}=0.0825$. This is just after the transition to the turbulent regime. Note $N L$ in the figure denotes $\left(\frac{\delta_{I}}{L}\right)_{W N}^{2}$. 


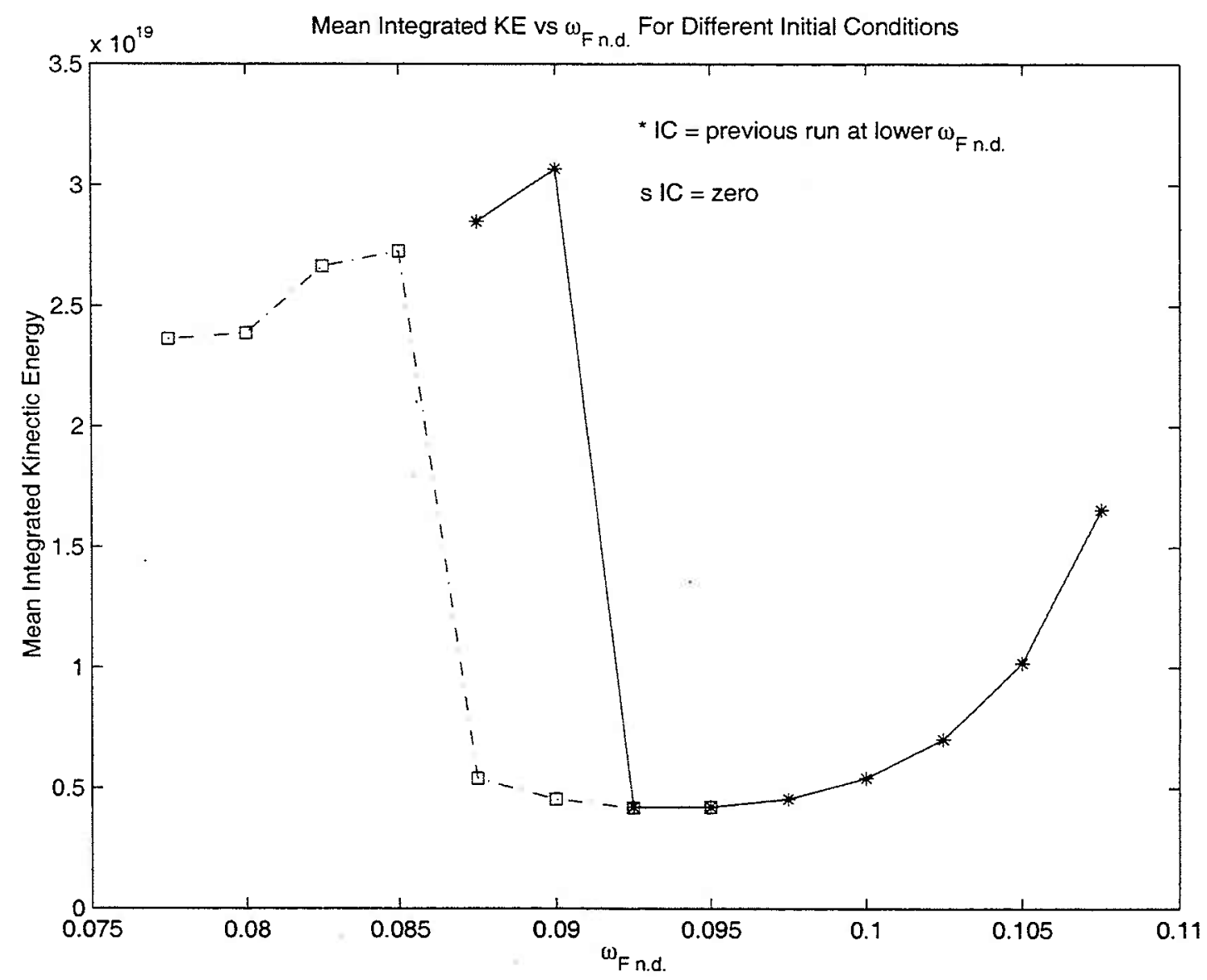

Figure B-22: Mean integrated kinetic energy vs nondimensional forcing frequency, $\omega_{F_{n . d .}}$. The results plotted by squares indicates that the solution was initialized with zero initial conditions. The results plotted with the asterisk indicate that each run was initialized with the results of the previous run forced at the lower forcing frequency. A hysteresis is observed for the forcing frequencies $\omega_{F_{n . d}}=0.085$ for zero initial conditions and $\omega_{F_{n . d .}}=0.09$ for initial conditions of lower frequency run. 
Envelope Contours of Runs Initialized by Results Forced at Lower Frequencies

(a) Initialized with $\omega_{F \text { n.d. }}=0.0875$

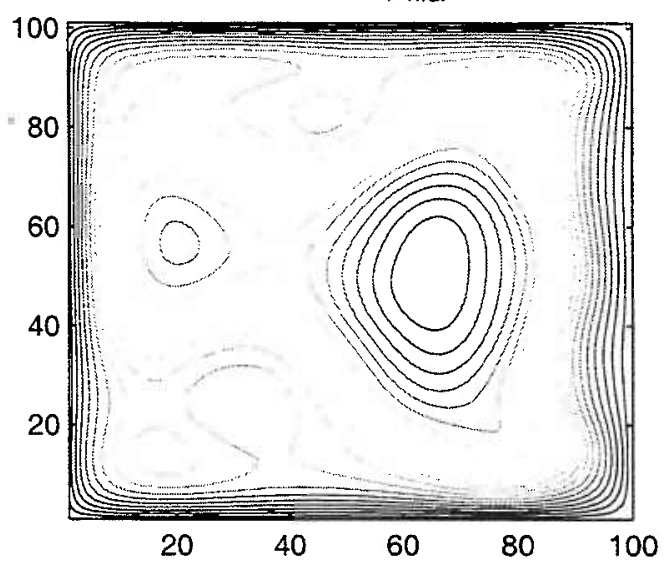

$\omega_{\mathrm{F} \mathrm{n.d.}}=0.09, \mathrm{NL}=0.01$ (b) Initialized with $\omega_{F \text { n.d. }}=0.09$

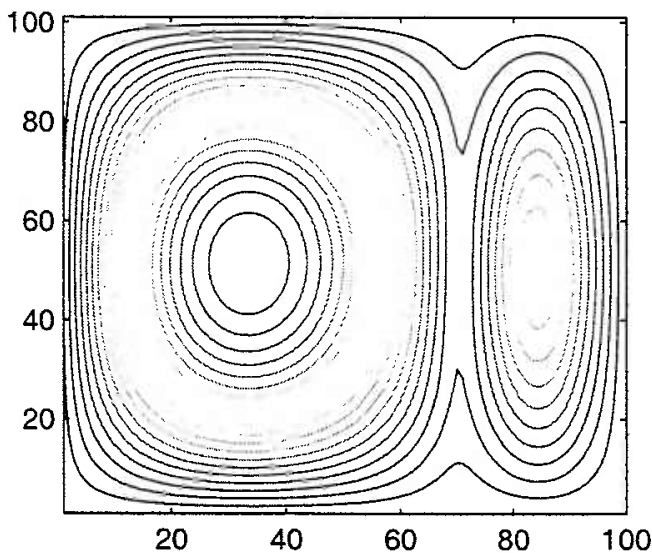

$\omega_{\text {F n.d. }}=0.0925, \mathrm{NL}=0.01$

Figure B-23: (a) is the RMS of the stream function just before the transition and (b) is the RMS of the stream function just after the transition when the solution is in its weakly nonlinear state. These runs have been initialized with solutions forced at a lower frequency. Note $N L$ in the figure denotes $\left(\frac{\delta_{I}}{L}\right)_{W N}^{2}$. 


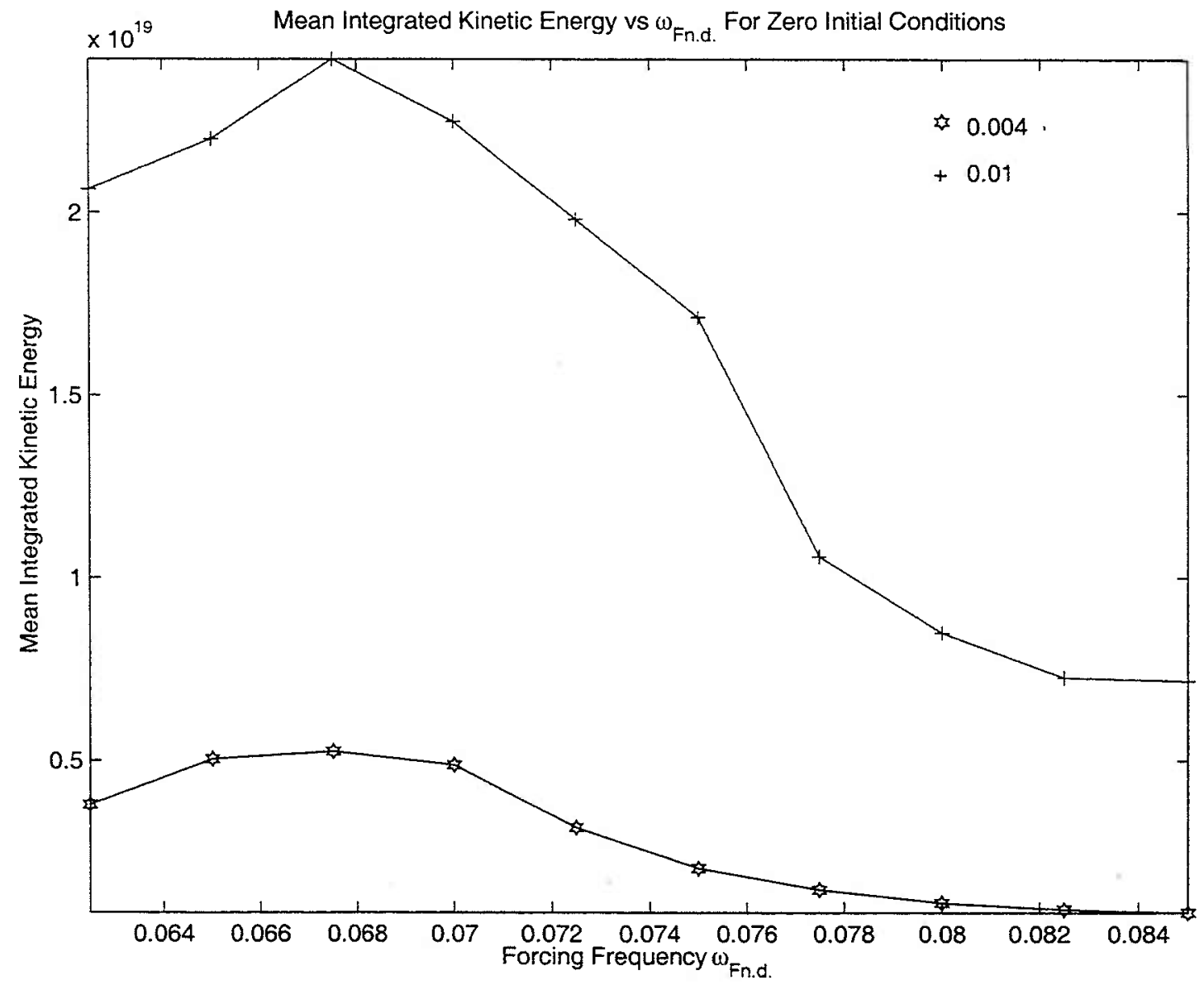

Figure B-24: Mean integrated kinetic energy over the area of the barrier basin vs forcing frequency for runs with forcing strengths of $\left(\frac{\delta_{I}}{L}\right)_{W N}^{2}=0.004$ and 0.01 respectively. All runs were done with zero initial conditions. 
Envelope for $\Psi$ for Forcing $\left(\delta_{\mathrm{l}} / \mathrm{L}\right)_{W N}^{2}=0.004$

(a)

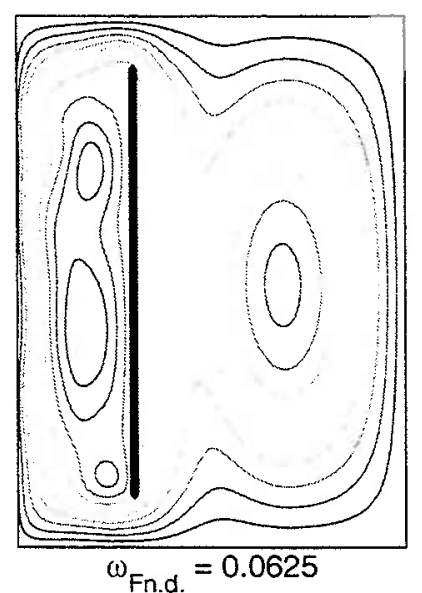

(d)

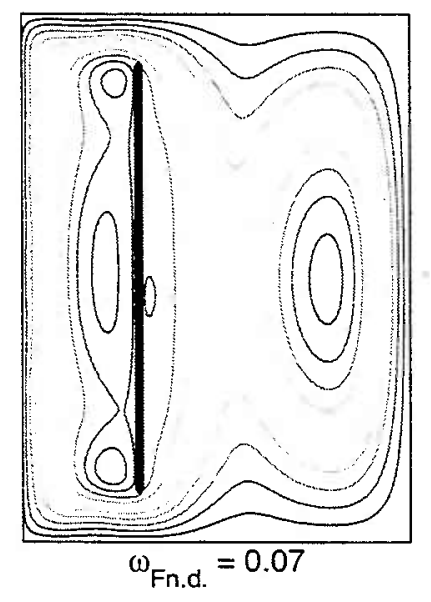

(g)

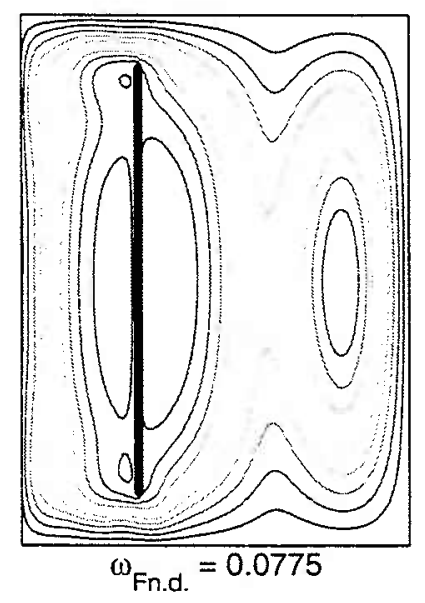

(b)

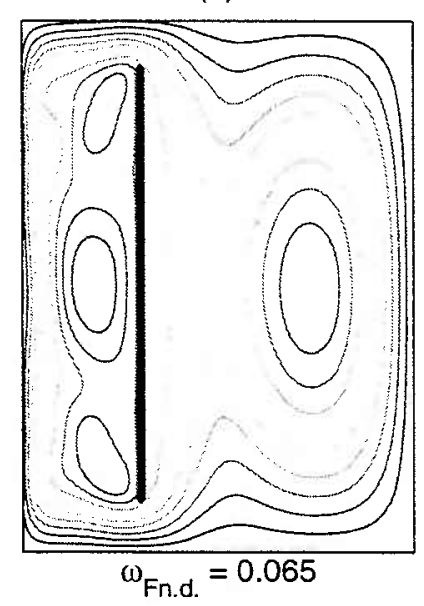

(e)

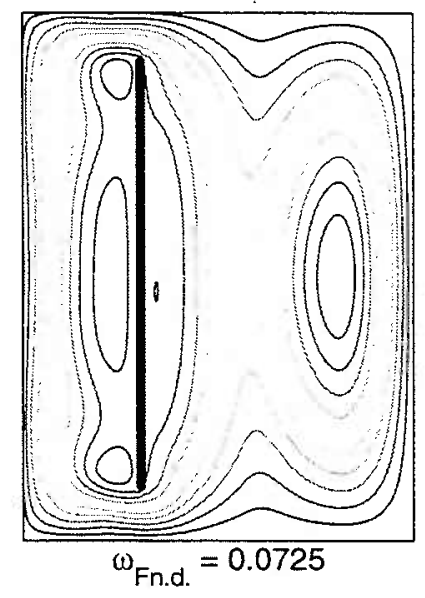

(h)

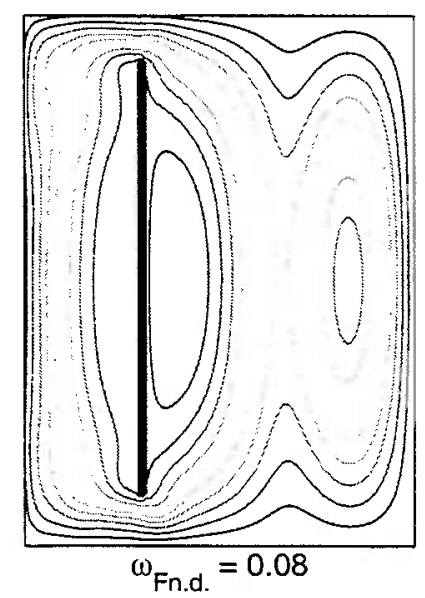

(c)

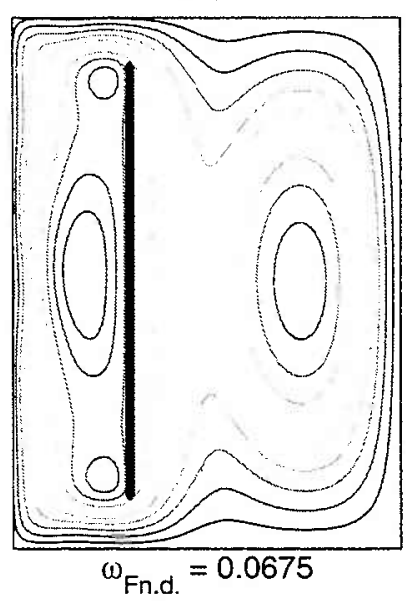

(f)

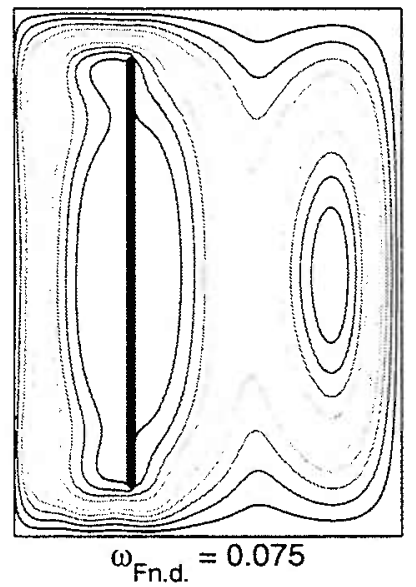

(i)

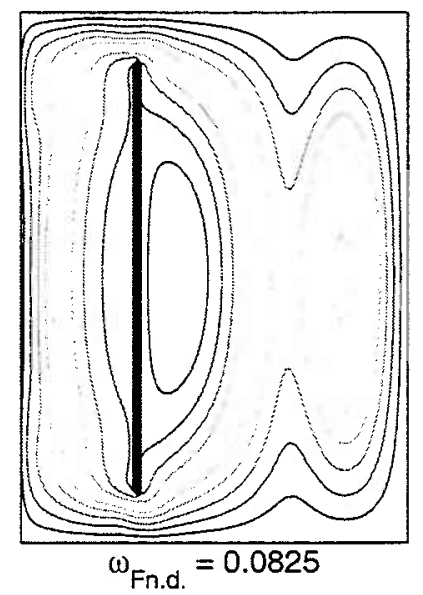

Figure B-25: The contours of the envelope function for all the solutions forced with a strength $\left(\frac{\delta_{I}}{L}\right)_{W N}^{2}=0.004$ and zero initial conditions. 
Envelope of $\Psi$ for Forcing $\left(\delta_{1} / L\right)_{W N}^{2}=0.01$

(a)

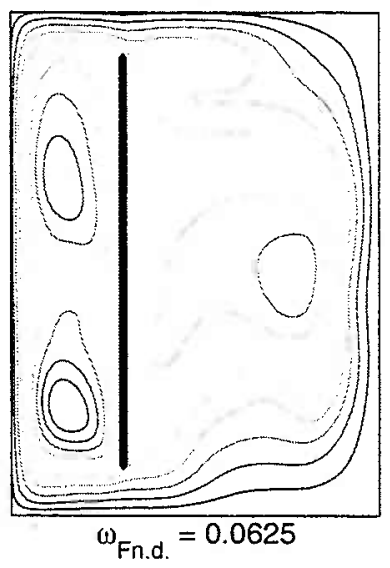

(d)

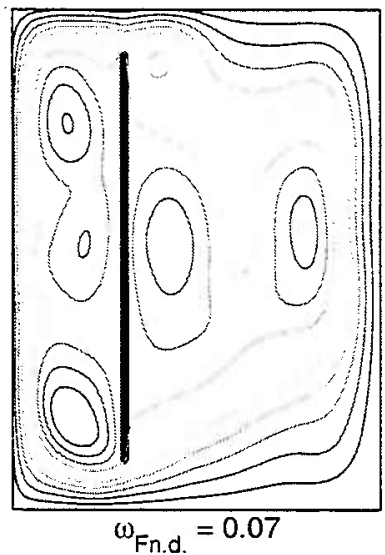

(g)

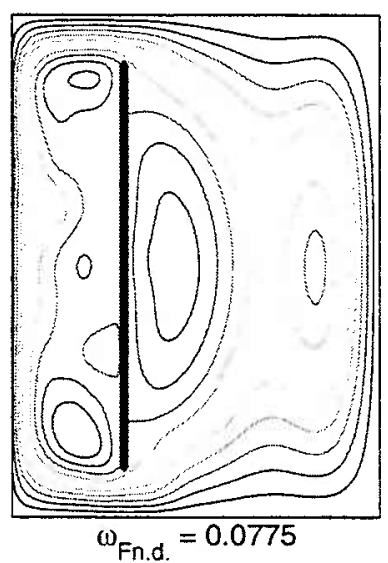

(b)

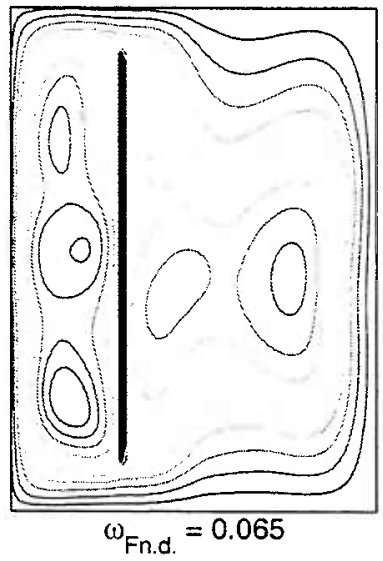

(e)

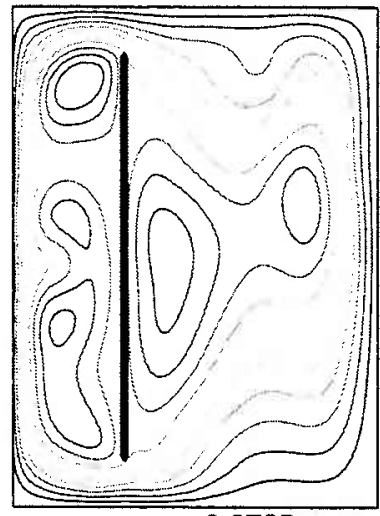

$\omega_{\text {Fn.d. }}=0.0725$

(h)

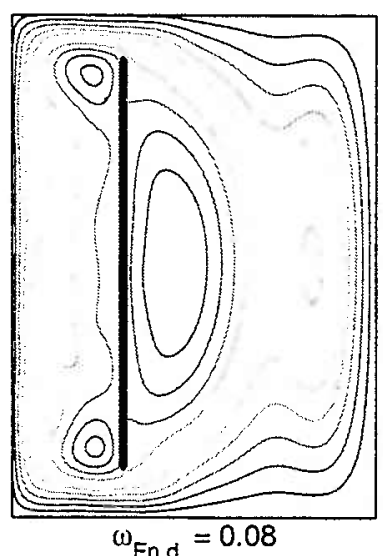

(c)

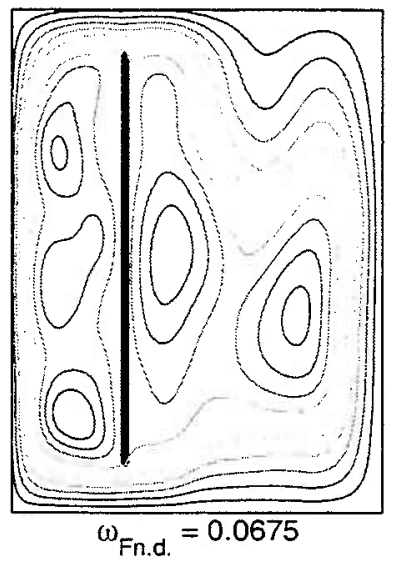

(f)

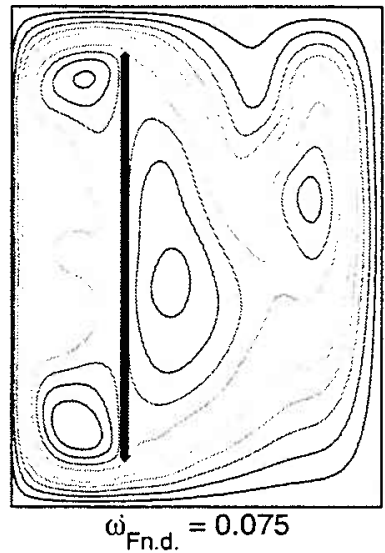

(i)

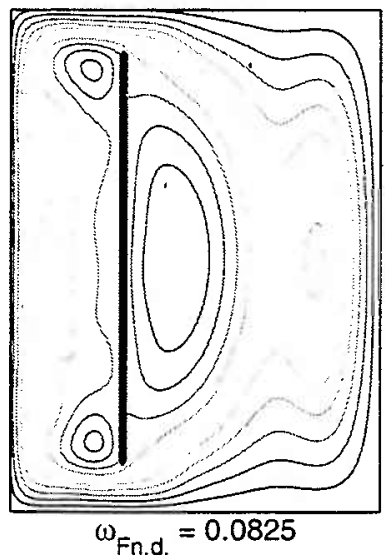

Figure B-26: The contours of the envelope function for all the solutions forced with a strength $\left(\frac{\delta_{I}}{L}\right)_{W N}^{2}=0.01$ and zero initial conditions. Note the slight change in the nonlinearity of the solution in the eastern basin between the frequencies $\omega_{F_{n . d}}=0.075$ and $\omega_{F_{n . d .}}=0.0775$. The solution forced with a frequency of $\omega_{F_{n . d .}}=0.0775$ looks more linear than the the solution forced at $\omega_{F_{n . d .}}=0.075$. 
Contours of $\Psi$ at 2 Day Intervals for $(\delta / L)_{W N}^{2}=0.004$ and $\omega_{F n . d .}=0.0725$

(a)

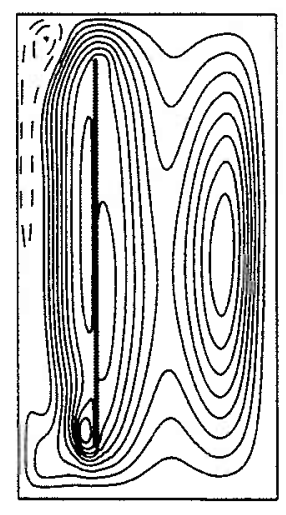

(e)

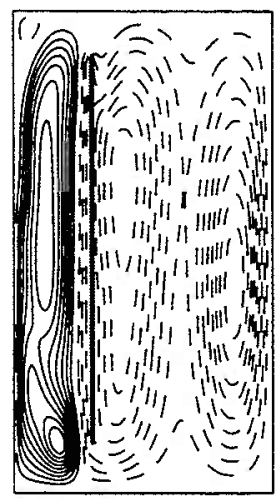

(i)

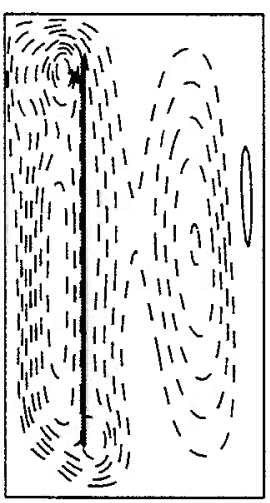

(b)

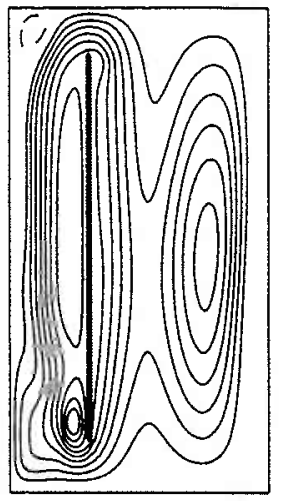

(f)

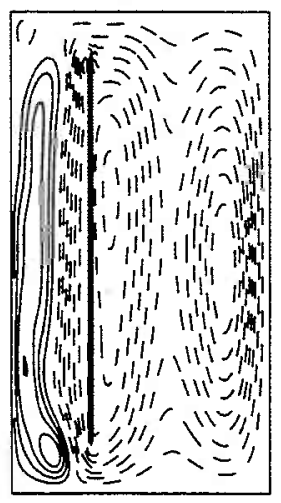

(j)

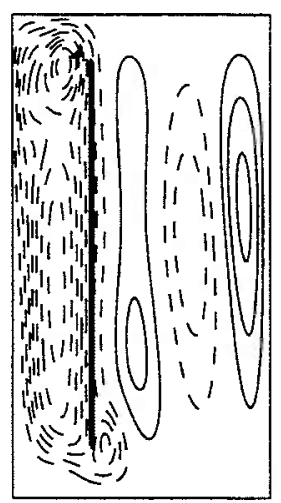

(c)

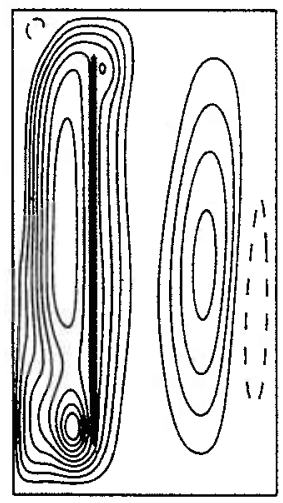

(g)

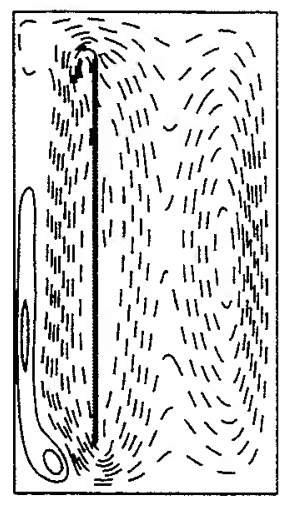

(h)

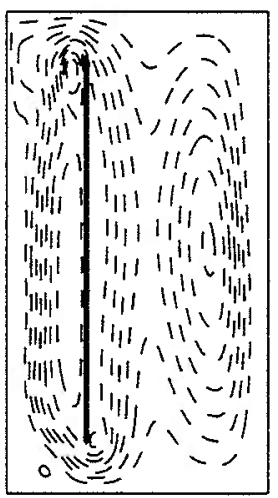

(d)

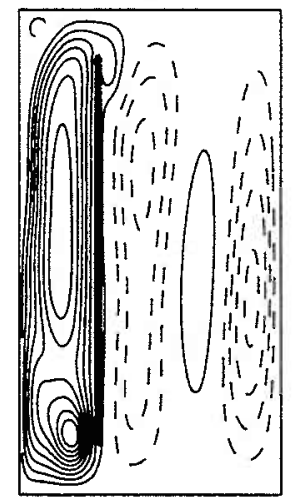

(k)

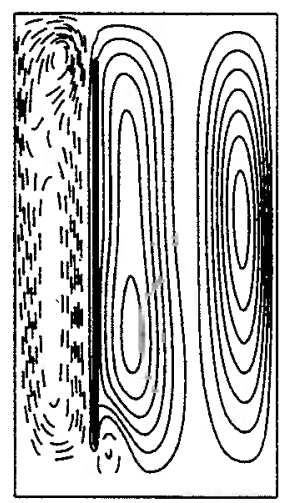

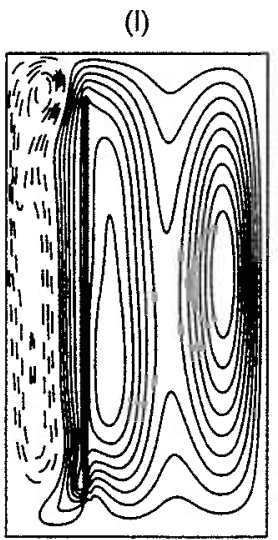

Figure B-27: Contours of the stream function, $\Psi$, at 2 days intervals for the solution $\left(\frac{\delta_{I}}{L}\right)_{W N}^{2}=0.004$ and $\omega_{F_{n . d .}}=0.0725$ in the barrier basin. The contours span 24 days of the 25.08 day forcing period. Solid lines correspond to positive stream lines and dotted lines correspond to negative stream lines. The contours document the westward propagation of the solution during one forcing period. 
Contours of $\psi$ at 2 Day Intervals for $\left(\delta_{1} / L\right)_{W N}^{2}=0.01$ and $\omega_{\text {Fn.d. }}$

(a)

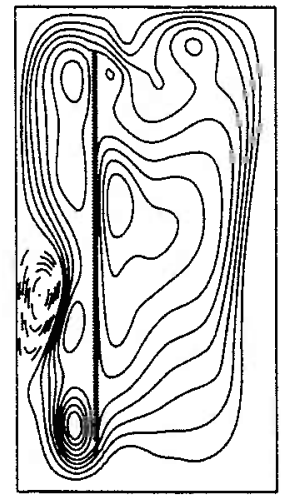

(e)

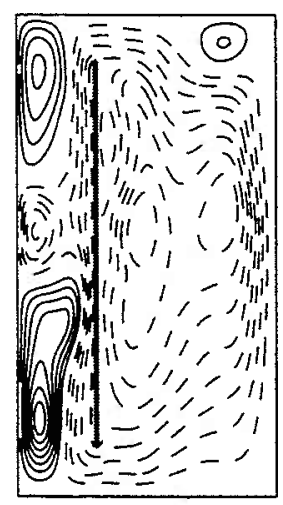

(i)

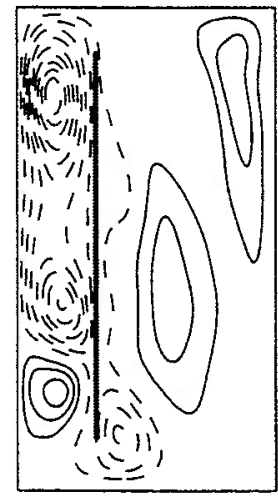

(b)

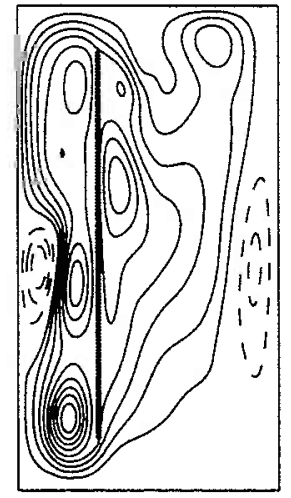

(f)

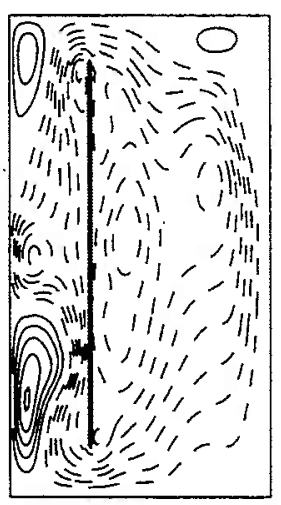

(j)

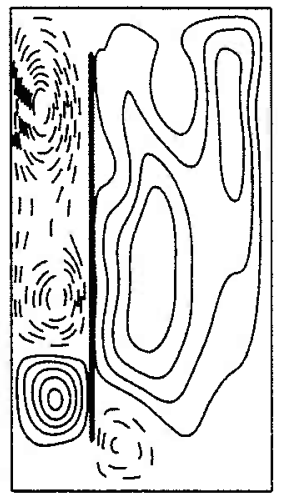

(c)

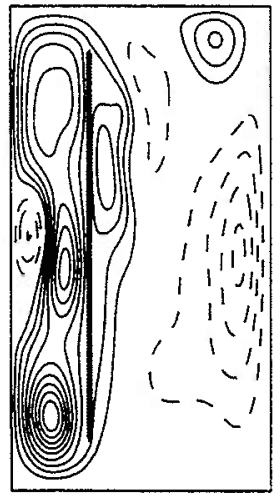

(g)

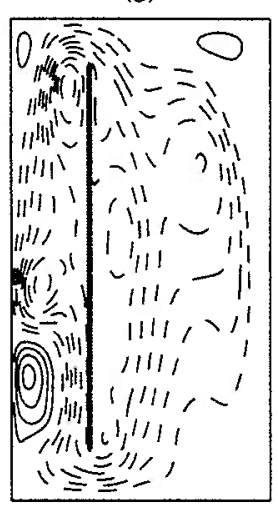

(k)

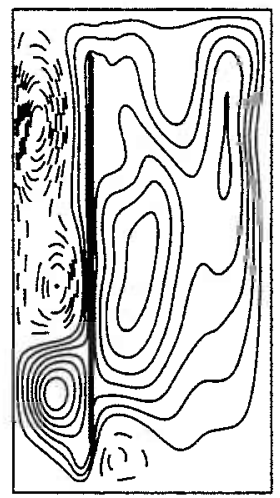

(d)

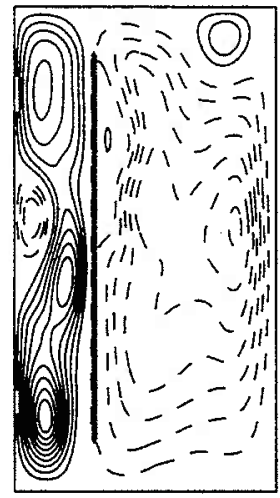

(h)

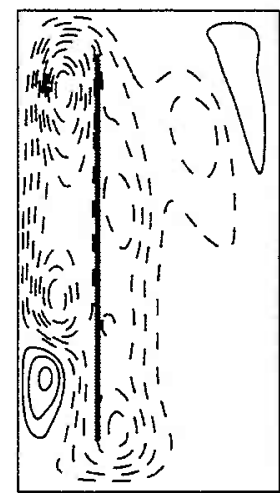

Figure B-28: Contours of the stream function, $\Psi$, at 2 days intervals for the solution $\left(\frac{\delta_{I}}{L}\right)_{W N}^{2}=0.01$ and $\omega_{F_{n . d} .}=0.0725$ in the barrier basin. The contours span 24 days of the 25.08 day forcing period. Solid lines correspond to positive stream lines and dotted lines correspond to negative stream lines. The contours document the westward propagation of the solution during one forcing period. 
Contours of $\Psi$ at 2 Day Intervals for $\left(\delta_{1}^{2} / L^{2}\right)_{W N}=0.01$ and $\omega_{F_{n . d .}}=0.0725$

(a)

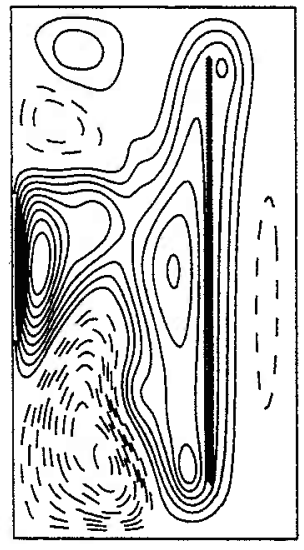

(b)

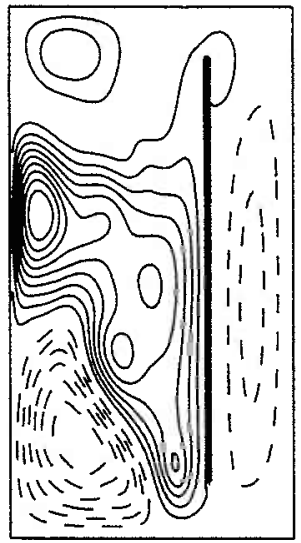

(c)

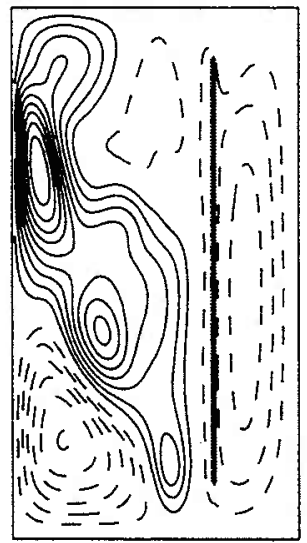

(d)

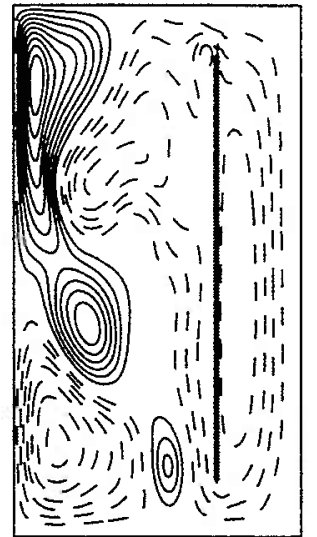

(e)

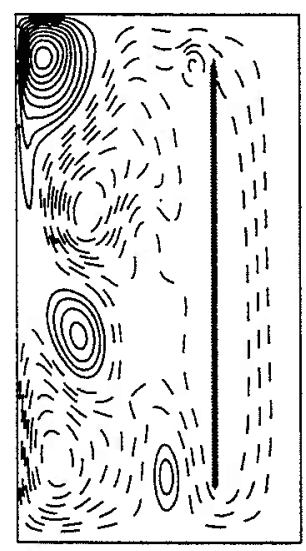

(f)

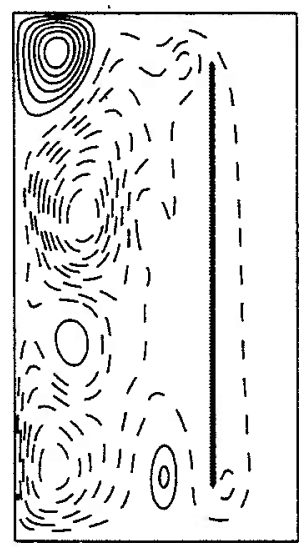

(g)

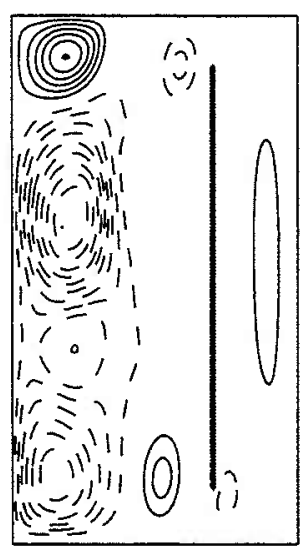

(h)

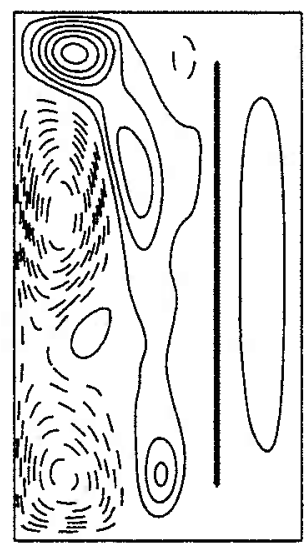

(i)

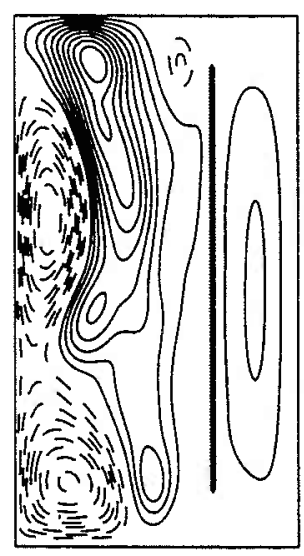

(j)

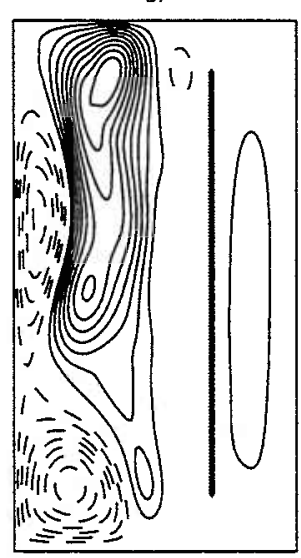

(k)

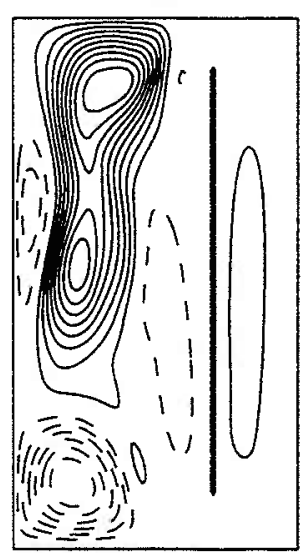

(I)

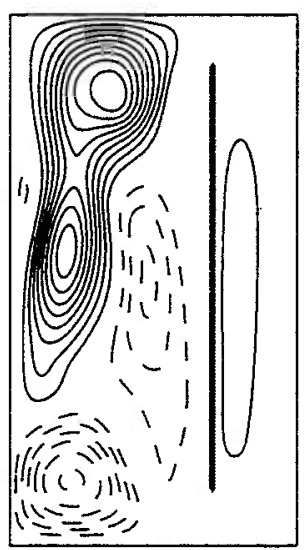

Figure B-29: Contour plots of $\Psi$ at two day intervals for the solution $\left(\frac{\delta_{I}}{L}\right)_{W N}^{2}=0.01$ and $\omega_{F_{n . d .}}=0.0725$. The barrier is placed at $1420 \mathrm{~km}$ to the east and the wind forcing is applied along a meridional strip from $200 \mathrm{~km}$ to $400 \mathrm{~km}$. 


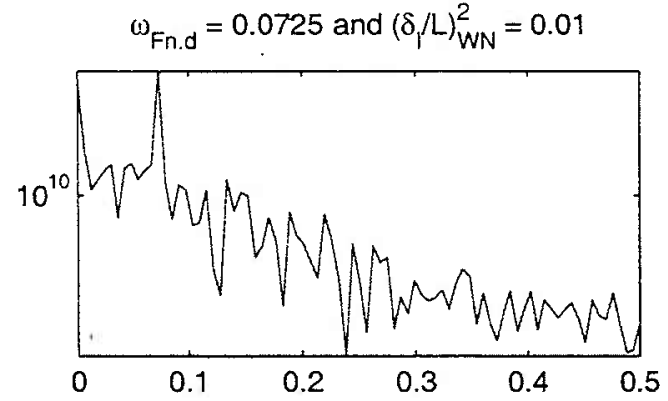

(a) $1000 \mathrm{~km} \mathrm{~N}, 200 \mathrm{~km} \mathrm{E}$

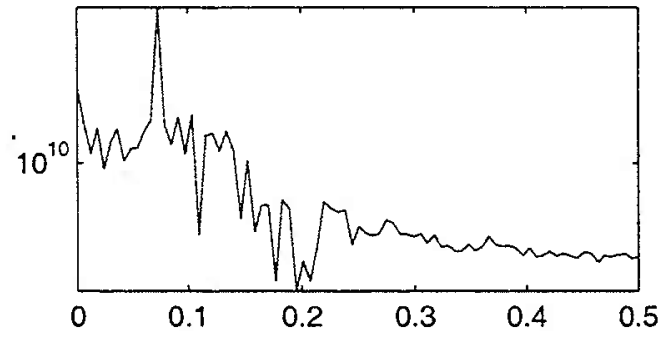

(c) $1000 \mathrm{~km} \mathrm{~N}, 1000 \mathrm{~km} \mathrm{E}$

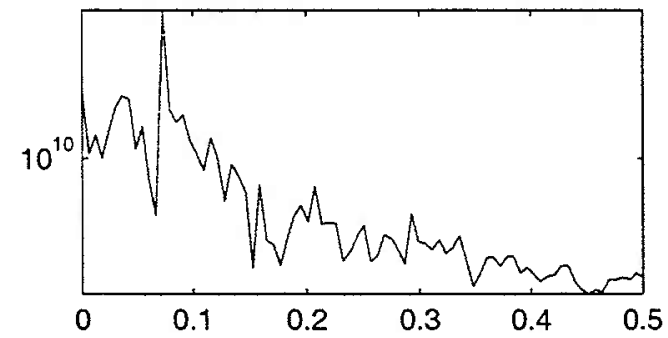

(e) $1000 \mathrm{~km} \mathrm{~N}, 1800 \mathrm{~km} \mathrm{E}$

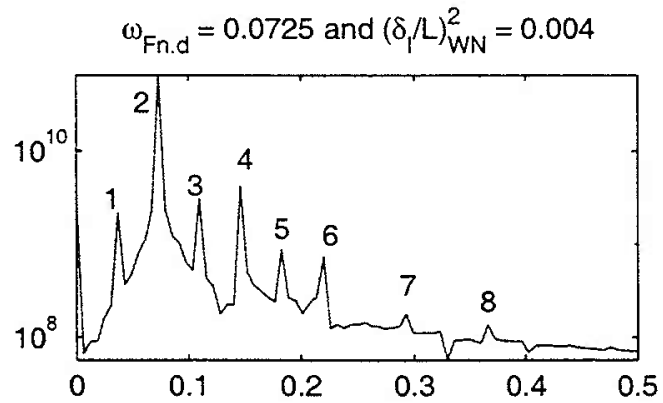

(b) $1000 \mathrm{~km} \mathrm{~N}, 200 \mathrm{~km} \mathrm{E}$

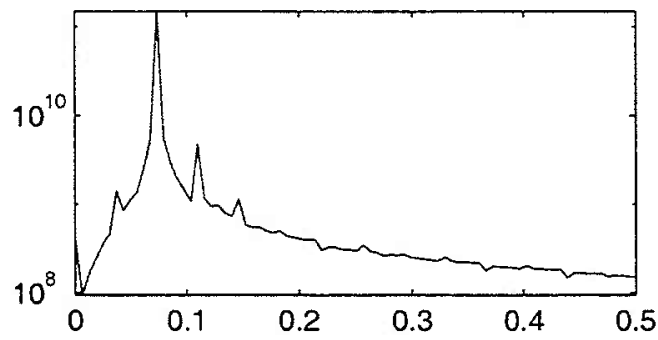

(d) $1000 \mathrm{~km} \mathrm{~N}, 1000 \mathrm{~km} \mathrm{E}$

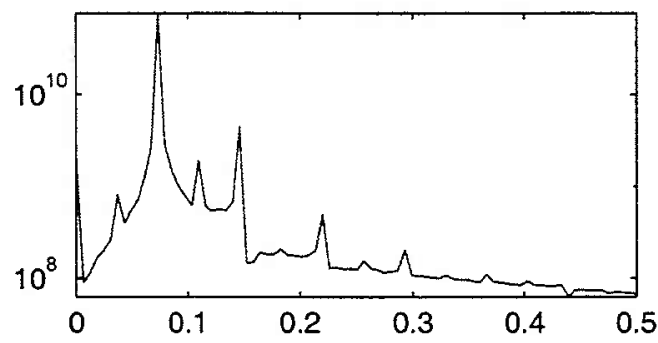

(f) $1000 \mathrm{~km} \mathrm{~N}, 1800 \mathrm{~km} \mathrm{E}$

Figure B-30: Fourier transform of the zonal velocity at points along the mid-latitude. (a), (c), and (e) are the solutions for $\left(\frac{\delta_{I}}{L}\right)_{W N}^{2}=0.01$ and $\omega_{F_{n . d .}}=0.0725$, while (b), (d), and (f) are the solutions for $\left(\frac{\delta_{I}}{L}\right)_{W N}^{2}=0.004$ and $\omega_{F_{n . d .}}=0.0725$. (a) and (b) are the Fourier transforms of velocities in the west of the basin, (c) and (d) are Fourier transforms in the middle, and (e) and (f) are Fourier transforms in the west of the basin. 

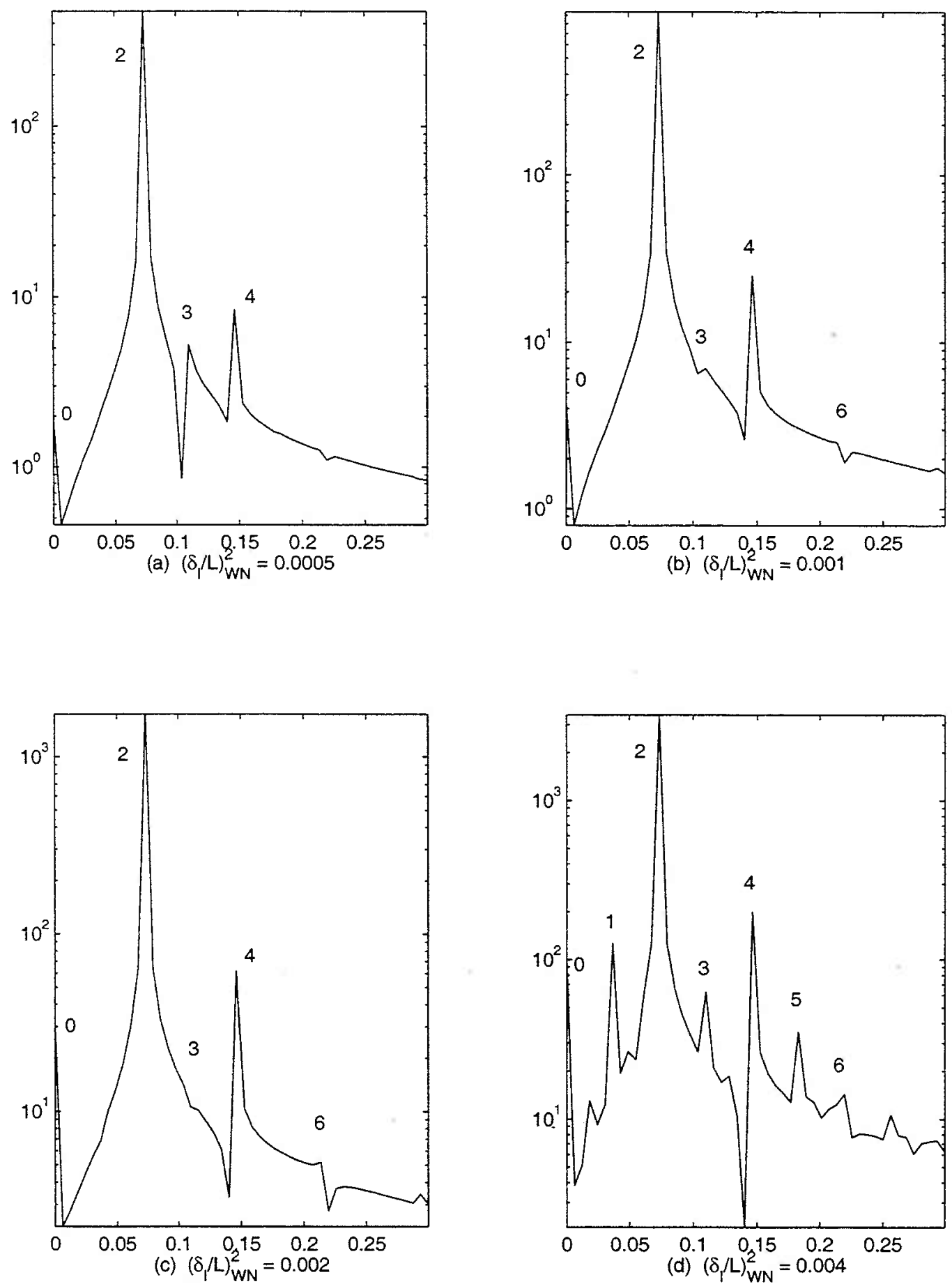

Figure B-31: Fourier transforms of the zonal velocity for solutions forced at 0.0725 and increasing wind strength. Figures (a), (b) and (c) indicate that as the forcing strength increases the first non-integer multiple of the forcing frequency to appear is at about 0.1 which is close to $\frac{3}{2}$ times $\omega_{F_{n . d .}}$ or the natural frequency of the first resonant mode in the barrier basin. 
Envelope Contours of Spatial Coefficients $\left(\delta_{/} / L\right)_{W N}^{2}=0.004$ and $\omega_{F_{n d}}=0.0725$

(a) peak 0

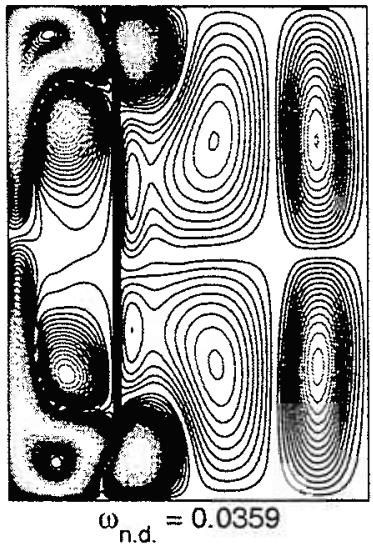

(d) peak 3

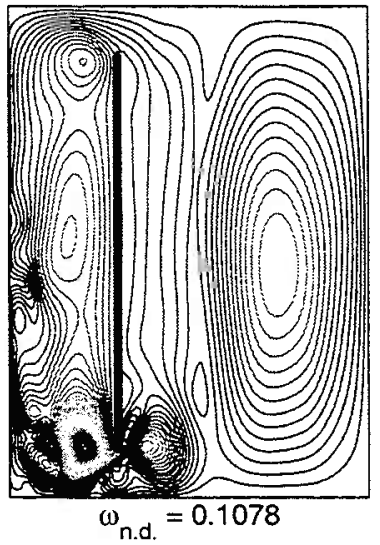

(g) peak 6

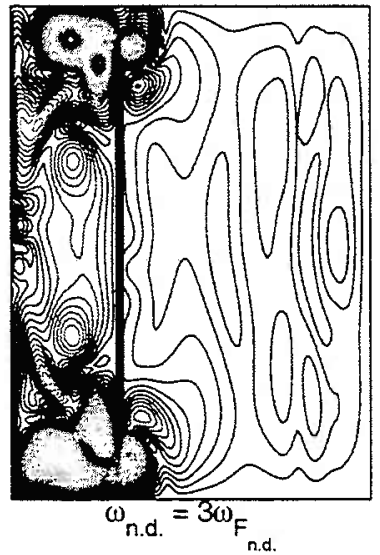

(b) peak 1

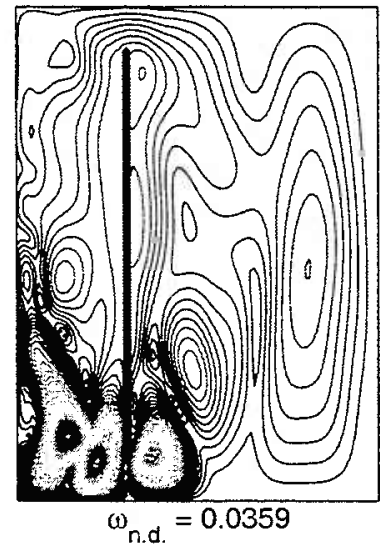

(e) peak 4

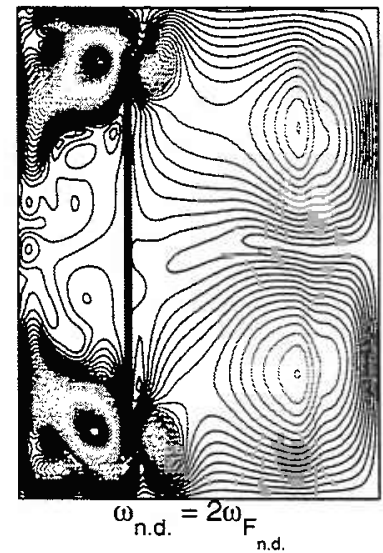

(h) peak 7

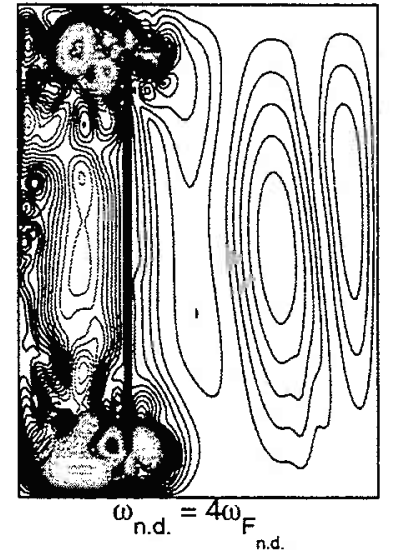

(c) peak 2

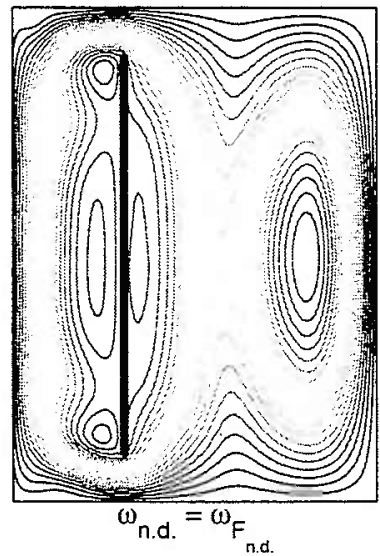

(f) peak 5

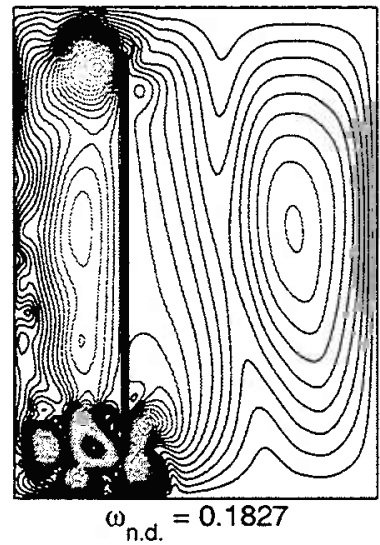

(i) peak 8

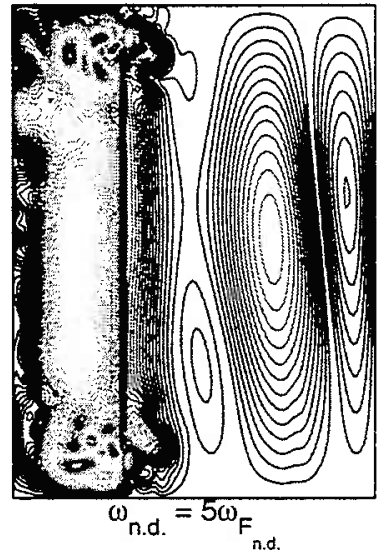

Figure B-32: Envelope plot for the spatial coefficients associated with the peaks labeled $1,2,3,4,5$, and 6 in Figure B-30 (a) corresponding to the solution $\left(\frac{\delta_{I}}{L}\right)_{W N}^{2}=$ 0.004 and $\omega_{F_{n . d .}}=0.0725$. 
Weakly Nonlinear Solution $\left(\delta_{l} / L\right)_{W N}^{2}=0.002$ and $\omega_{F_{\text {n. }}}=0.0725$

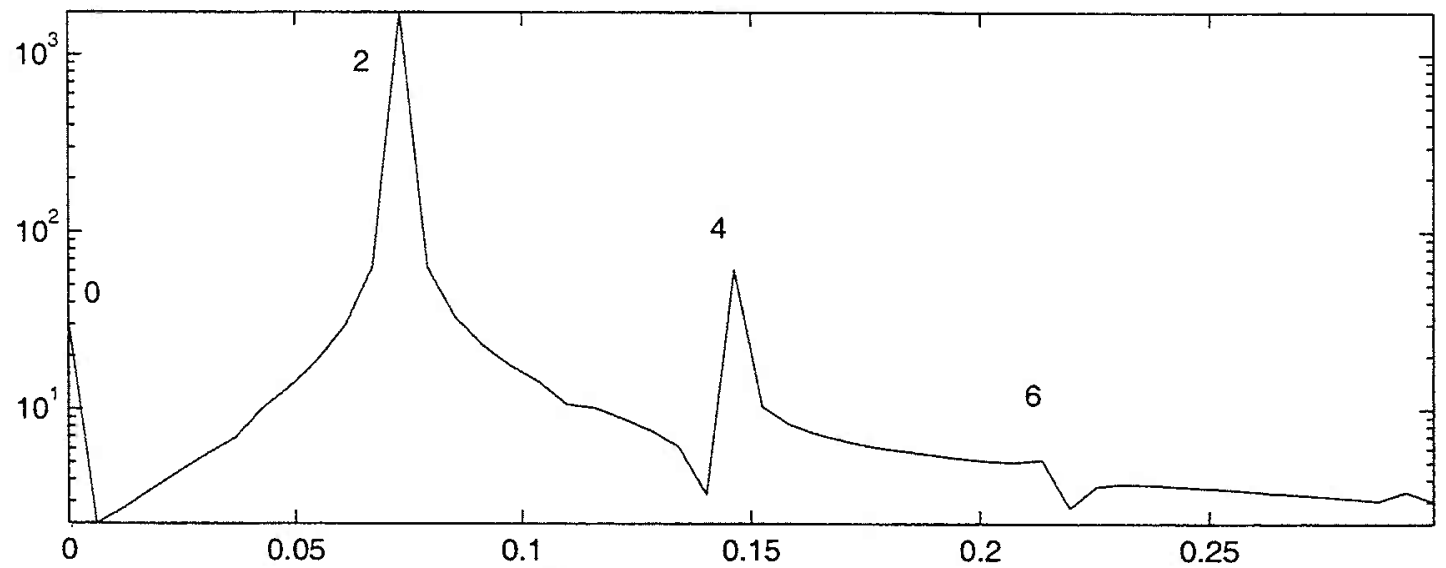

(a) Fourier Transform of Zonal Velocity

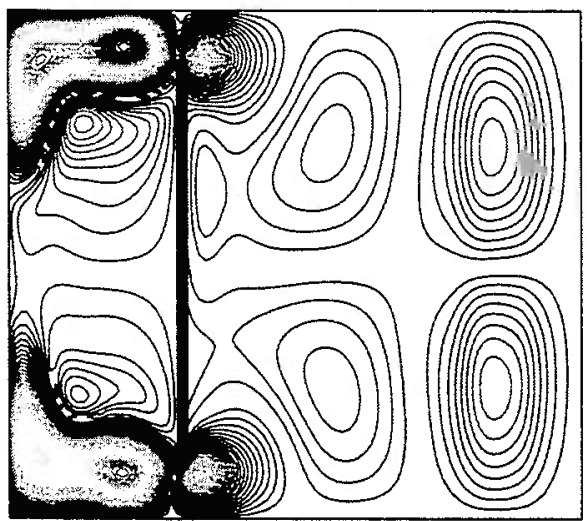

(b) $\omega_{\text {n.d. }}=0-$ peak 0

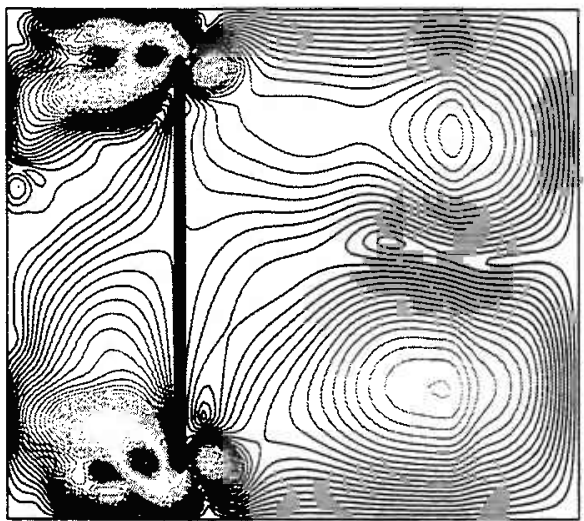

(d) $\omega_{\text {n.d. }}=0.145-$ peak 4

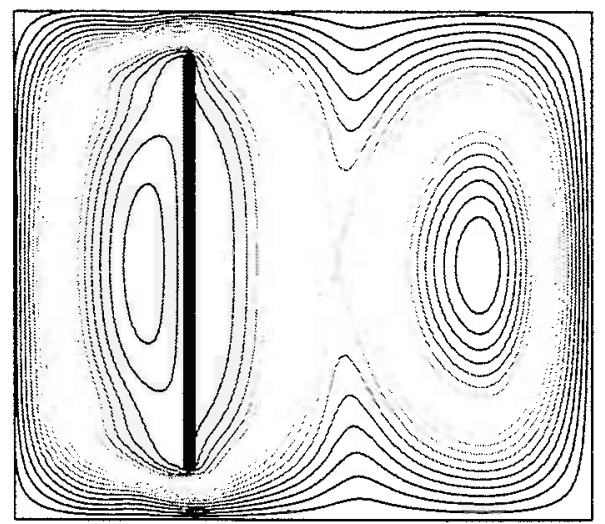

(c) $\omega_{\text {n.d. }}=0.0725$ - peak 2

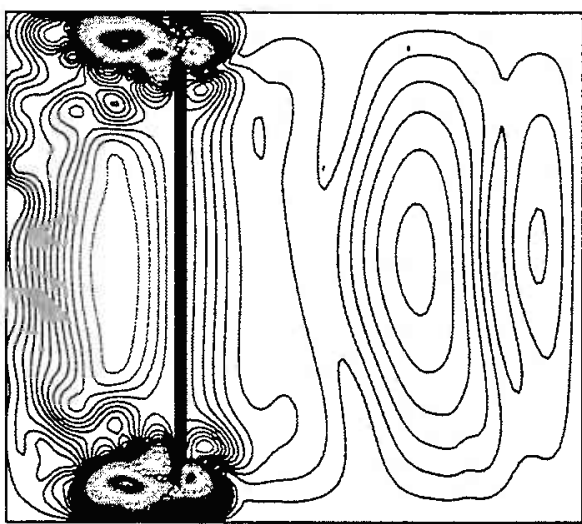

(e) $\omega_{\text {n.d. }}=0.2175-$ peak 6

Figure B-33: (a) plots the Fourier transform of the zonal velocity at a point. The peak labels, 0, 2, 4, and 6, correspond to the labels in Figure B-30 (b). (b), (c), (d), and (e) above are the absolute values of the spatial coefficients corresponding to peaks $0,2,4$, and 6 labeled in (a). 
Weakly Nonlinear Solution $\left(\delta_{\mathrm{l}} / \mathrm{L}\right)_{\mathrm{WN}}^{2}=0.002$ and $\omega_{F_{\text {n.d. }}}$

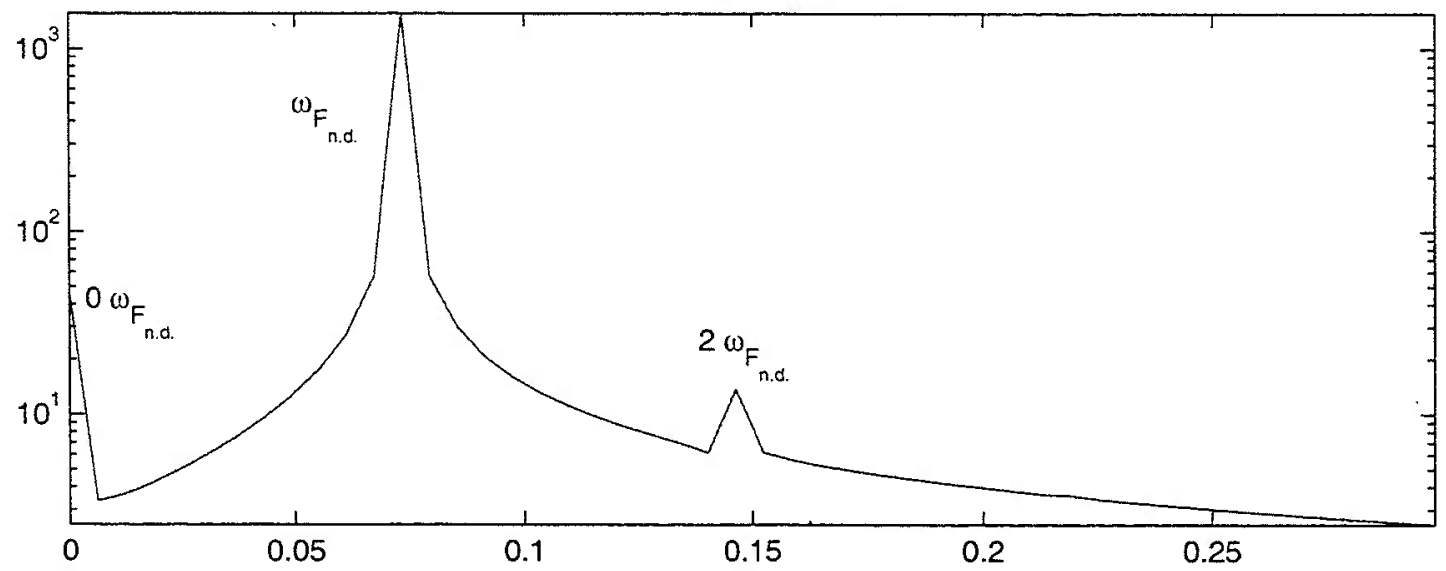

(a) Fourier Transform of Zonal Velocity
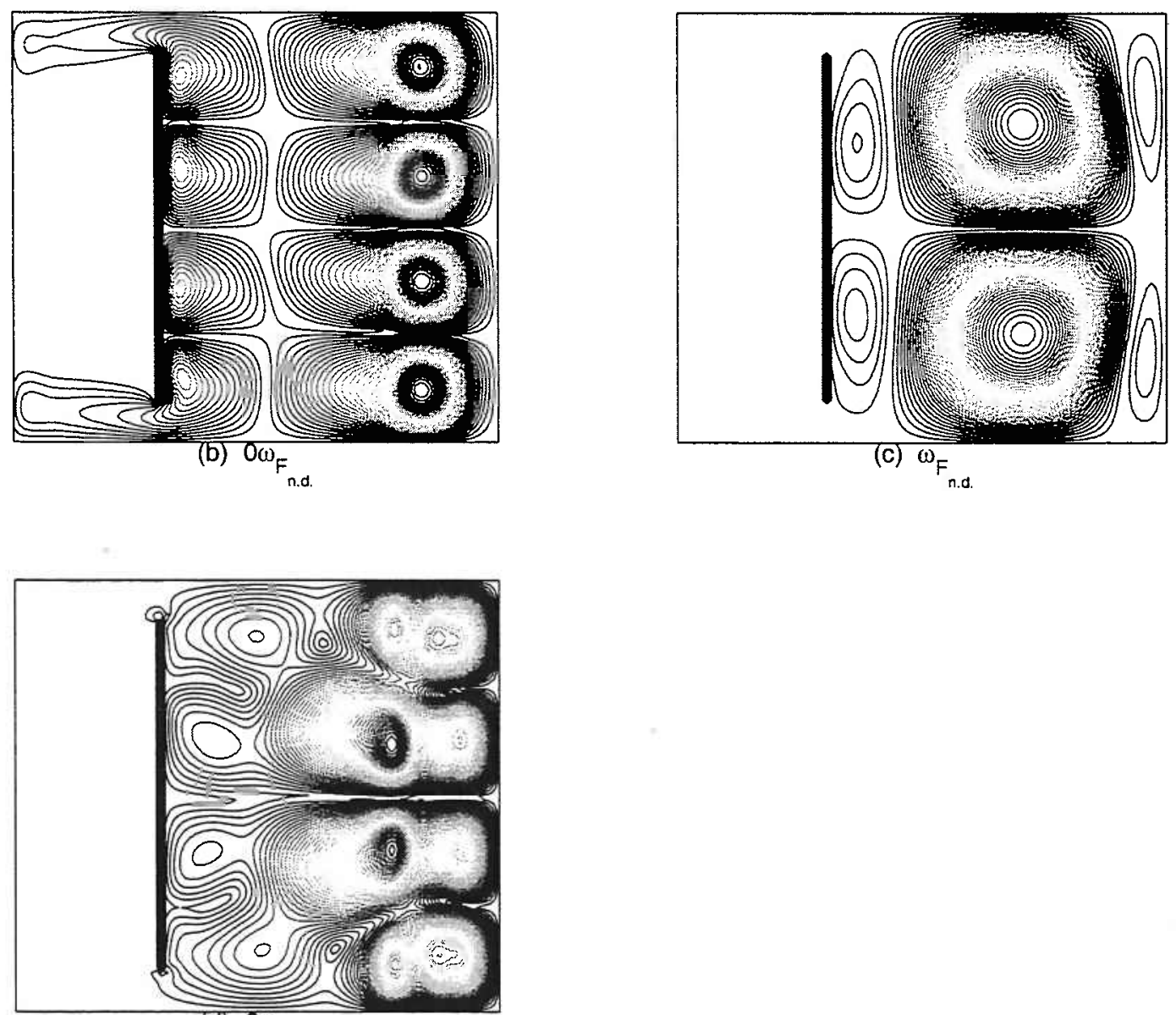

(d) $2 \omega_{F}$ n.d.

Figure B-34: (a) plots the Fourier transform of the zonal velocity at a point. The peak are labeled correspond their relation to the forcing frequency, $\omega_{F_{n . d . *}}$ (b), (c), and (d) above are the absolute values of the spatial coefficients corresponding to peaks labeled in (a). 


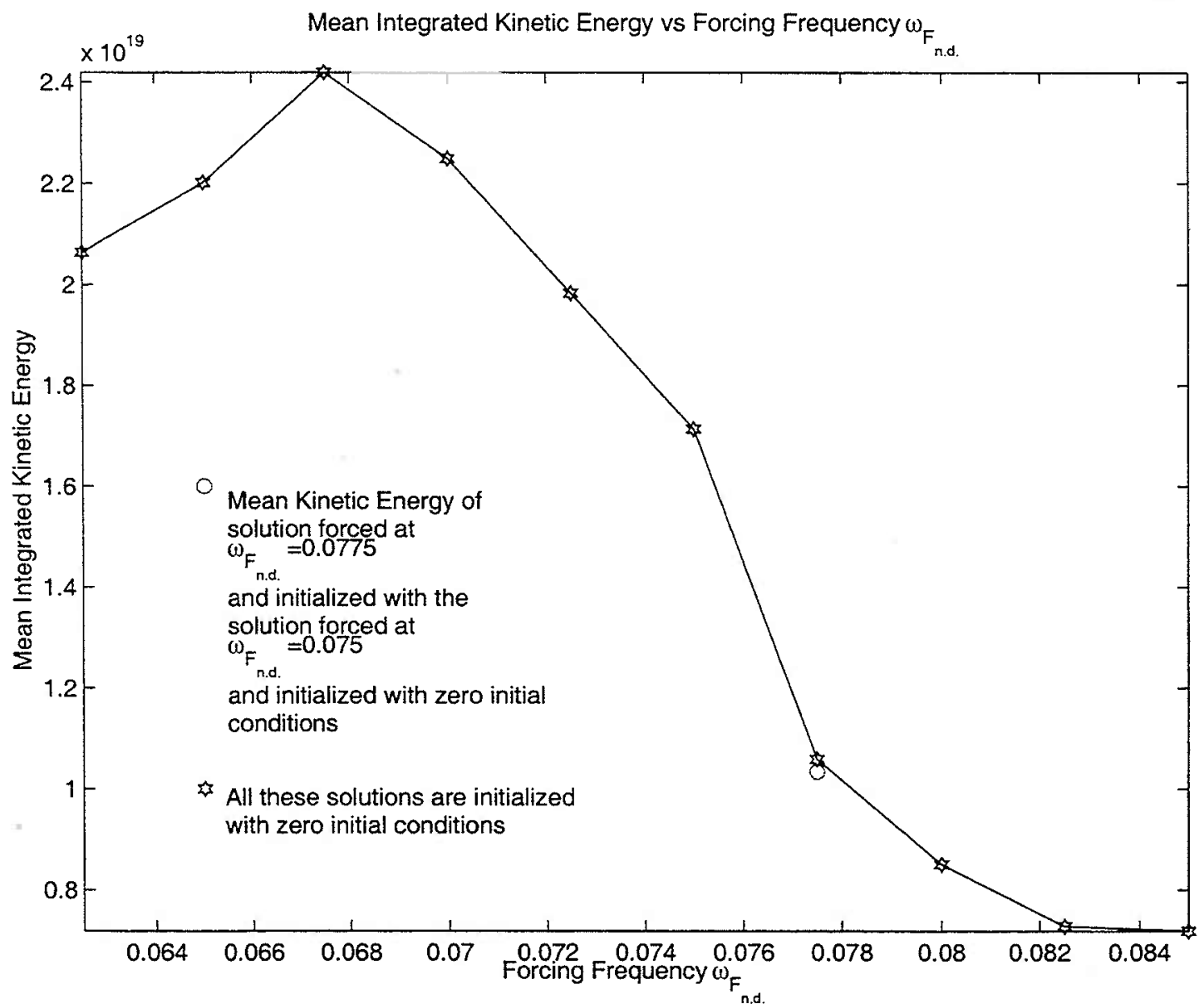

Figure B-35: Mean integrated kinetic energy for the solutions forced with a strength of $\left(\frac{\delta_{I}}{L}\right)_{W N}^{2}=0.01$ in the barrier basin. The six pointed star represents solutions initialized with zero initial conditions and the circle represents the solution forced at $\omega_{F_{n . d .}}=0.0775$ initialized with the solution of $\omega_{F_{n . d .}}=0.075$. 


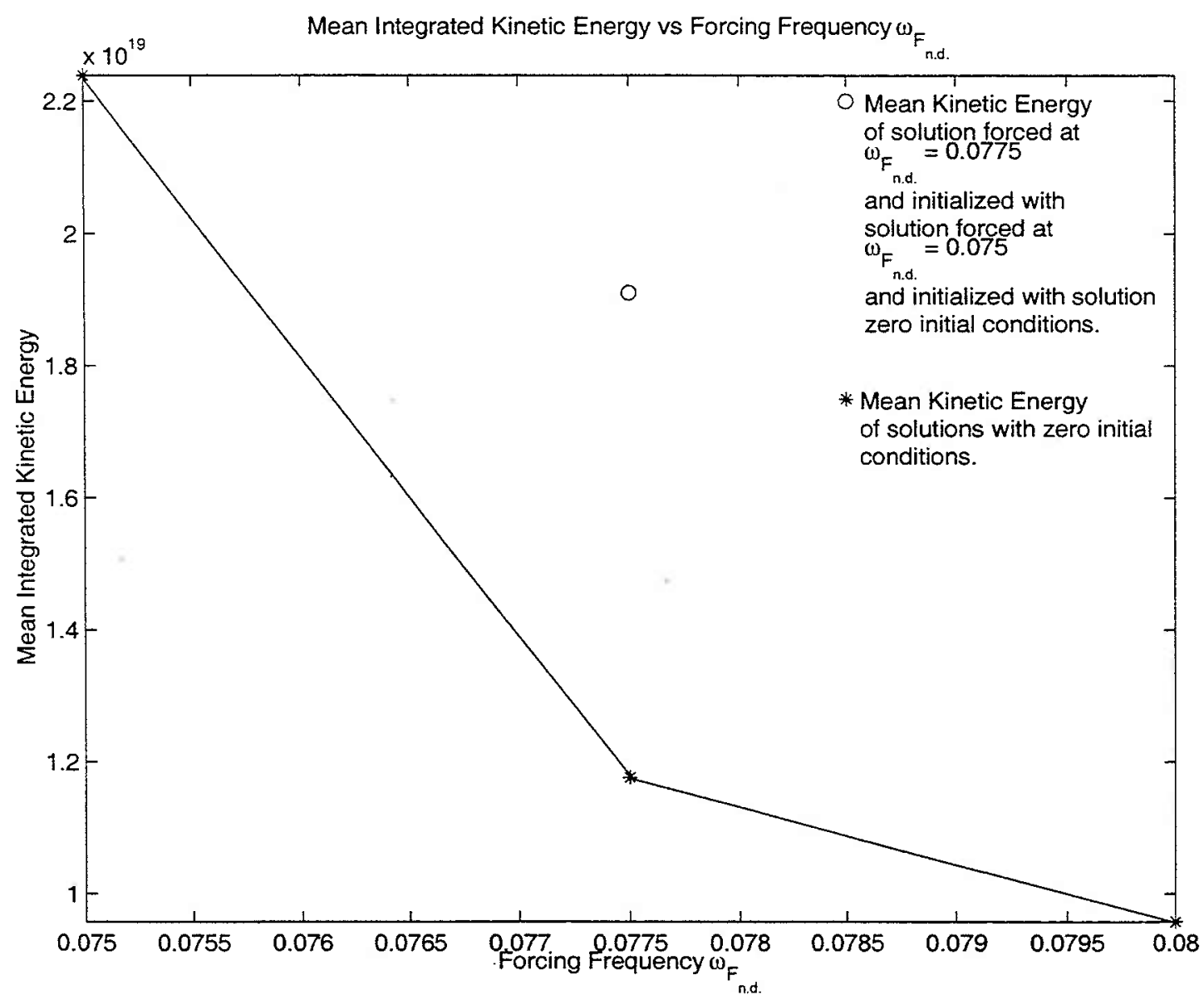

Figure B-36: The asterisks, ${ }^{*}$, represent the mean integrated kinetic energy of the solution initialized with zero initial conditions for a wind forcing of $\left(\frac{\delta_{I}}{L}\right)_{W N}^{2}=0.01$ and bottom friction $\frac{\delta_{S}}{L}=0.001$. The circles, $(0)$, represent the mean integrated kinetic energy of the solution forced with a nondimensional frequency of $\omega_{F_{n . d .}}=0.0775$ and initialized with the solution which was forced at $\omega_{F_{n . d .}}=0.075$. 


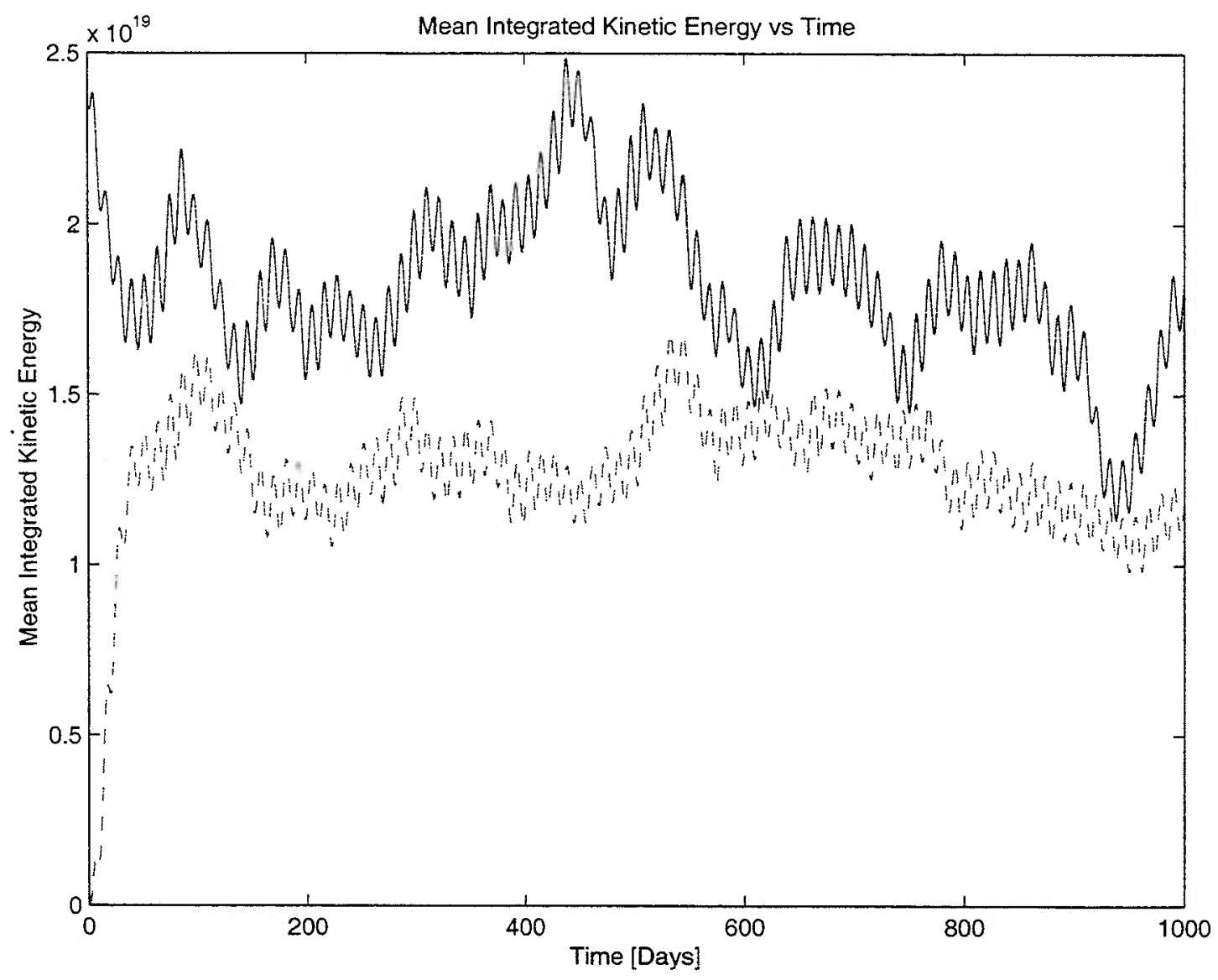

Figure B-37: The dotted line plots the mean integrated kinetic energy vs the time in model days of the run forced with $\omega_{F_{n . d .}}=0.0775,\left(\frac{\delta_{L}}{L}\right)_{W N}^{2}=0.01$ and $\frac{\delta_{S}}{L}=0.001$ and initialized with zero initial conditions. The solid line represents the corresponding run initialized with the solution from the lower forcing frequency $\omega_{F_{n . d .}}=0.075$. 
Envelope Contours of Solutions With $\omega_{F_{n . d .}}=0.0775$ and $\left(\delta_{1}^{2} / L^{2}\right)_{W N}=0.01$

Solution forced with zero initial conditions.

(a)

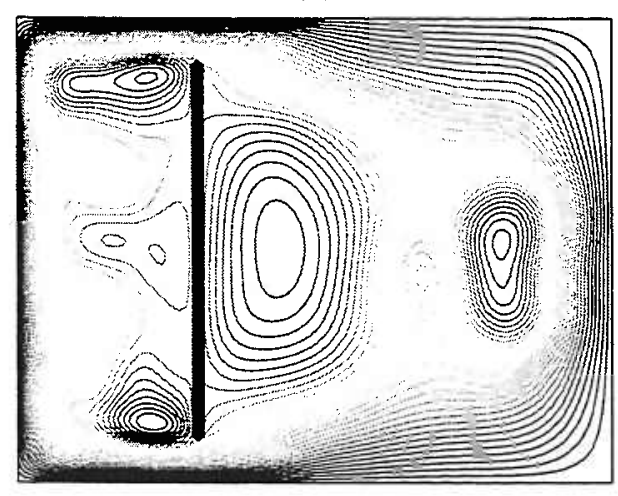

Solution forced using the solution which was forced at

$\omega_{F}$

as initial conditions. (b)

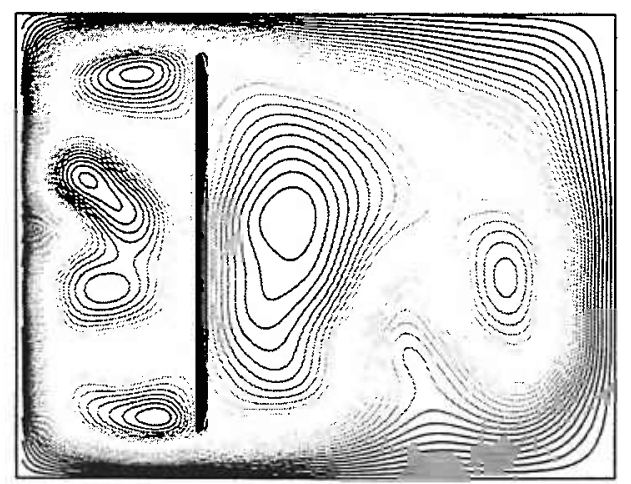

Figure B-38: Contour plots of envelope for solutions forced at $\left(\frac{\delta_{I}}{L}\right)_{W N}^{2}=0.01, \frac{\delta_{S}}{L}=$ 0.001 , and $\omega_{F_{n . d .}}=0.0775$. (a) initialized with zero initial conditions and (b) initialized with the solution forced at $\omega_{F_{n . d .}}=0.075$. 


\section{Appendix C}

\section{Analytical Expression for Variance in the Linear Barrier Basin}

\section{Problem}

The following are the full expressions for the variance for the full basin, the variance for the western sub-basin, and the variance for the eastern sub-basin, where $a$ is the real part of $\alpha_{n}, b$ is the imaginary part of $\alpha_{n}$, and $c=\frac{\gamma}{\left(\omega^{2}+\gamma^{2}\right)}$.

$$
\begin{aligned}
& \mathrm{FV}=\frac{e^{c x_{I}}}{2} \sum_{n=1}^{\infty}\left\{\frac { | E _ { n } | ^ { 2 } } { 4 } \quad \left[e^{-c x_{I}}\left(\frac{e^{2 a x_{I}}}{2 a-c}+\frac{e^{-2 a x_{I}}}{-2 a-c}-\frac{e^{2 i b x_{I}}}{2 i b-c}-\frac{e^{-2 i b x_{I}}}{-2 i b-c}\right)\right.\right. \\
&\left.-\left(\frac{1}{2 a-c}+\frac{1}{-2 a-c}-\frac{1}{2 i b-c}-\frac{1}{-2 i b-c}\right)\right]+ \\
& \frac{\left|C_{n}\right|^{2}}{4} \quad {\left[e^{-c x_{F}}\left(\frac{e^{-2 a\left(x_{I}-x_{F}\right)}}{2 a-c}+\frac{e^{2 a\left(x_{I}-x_{F}\right)}}{-2 a-c}-\frac{e^{-2 i b\left(x_{I}-x_{F}\right)}}{2 i b-c}-\frac{e^{2 i b\left(x_{I}-x_{F}\right)}}{-2 i b-c}\right)\right.} \\
&\left.-e^{-c x_{I}}\left(\frac{1}{2 a-c}+\frac{1}{-2 a-c}-\frac{1}{2 i b-c}-\frac{1}{-2 i b-c}\right)\right]+ \\
& \frac{C_{n}^{*} D_{n}}{4} \quad {\left[e^{-c x_{F}}\left(\frac{e^{-2 a\left(x_{I}-x_{F}\right)}}{2 a-c}+\frac{e^{2 a\left(x_{I}-x_{F}\right)}}{-2 a-c}-\frac{e^{2 i b\left(x_{I}-x_{F}\right)}}{-2 i b-c}-\frac{e^{-2 i b\left(x_{I}-x_{F}\right)}}{2 i b-c}\right)\right.} \\
&\left.-e^{-c x_{I}}\left(\frac{1}{2 a-c}+\frac{1}{-2 a-c}+\frac{1}{-2 i b-c}-\frac{1}{2 i b-c}\right)\right]+ \\
& \frac{C_{n} D_{n}^{*}}{4} \quad\left[e^{-c x_{F}}\left(\frac{e^{-2 a\left(x_{I}-x_{F}\right)}}{2 a-c}+\frac{e^{2 a\left(x_{I}-x_{F}\right)}}{-2 a-c}-\frac{e^{-2 i b\left(x_{I}-x_{F}\right)}}{2 i b-c}-\frac{e^{2 i b\left(x_{I}-x_{F}\right)}}{-2 i b-c}\right)\right. \\
&\left.-e^{-c x_{I}}\left(\frac{1}{2 a-c}+\frac{1}{-2 a-c}+\frac{1}{2 i b-c}-\frac{1}{-2 i b-c}\right)\right]+ \\
& \frac{\left|D_{n}\right|^{2}}{4} \quad {\left[e^{-c x_{F}}\left(\frac{e^{-2 a\left(x_{I}-x_{F}\right)}}{2 a-c}+\frac{e^{2 a\left(x_{I}-x_{F}\right)}}{-2 a-c}+\frac{e^{2 i b\left(x_{I}-x_{F}\right)}}{-2 i b-c}+\frac{e^{-2 i b\left(x_{I}-x_{F}\right)}}{2 i b-c}\right)\right.} \\
&\left.-e^{-c x_{I}}\left(\frac{1}{2 a-c}+\frac{1}{-2 a-c}+\frac{1}{-2 i b-c}-\frac{1}{2 i b-c}\right)\right]+ \\
& \frac{\left|B_{n}\right|^{2}}{4} \quad {\left[-e^{-c x_{F}}\left(\frac{e^{2 a\left(x_{F}-1\right)}}{2 a-c}+\frac{e^{-2 a\left(x_{F}-1\right)}}{-2 a-c}-\frac{e^{2 i b\left(x_{F}-1\right)}}{2 i b-c}-\frac{e^{-2 i b\left(x_{F}-1\right)}}{-2 i b-c}\right)\right.} \\
&\left.\left.+e^{-c}\left(\frac{1}{2 a-c}+\frac{1}{-2 a-c}-\frac{1}{2 i b-c}-\frac{1}{-2 i b-c}\right)\right]\right\}
\end{aligned}
$$




$$
\begin{aligned}
\mathrm{WV}=\frac{e^{c x_{I}}}{2} \sum_{n=1}^{\infty}\left\{\frac{\left|E_{n}\right|^{2}}{4}\right. & {\left[e^{-c x_{I}}\left(\frac{e^{2 a x_{I}}}{2 a-c}+\frac{e^{-2 a x_{I}}}{-2 a-c}-\frac{e^{2 i b x_{I}}}{2 i b-c}-\frac{e^{-2 i b x_{I}}}{-2 i b-c}\right)\right.} \\
& \left.\left.-\left(\frac{1}{2 a-c}+\frac{1}{-2 a-c}-\frac{1}{2 i b-c}-\frac{1}{-2 i b-c}\right)\right]\right\}
\end{aligned}
$$

$$
\begin{aligned}
\mathrm{EV}=\frac{e^{c x_{I}}}{2} \sum_{n=1}^{\infty}\left\{\begin{aligned}
\left.|| C_{n}\right|^{2} \\
4
\end{aligned}\right. & {\left[e^{-c x_{F}}\left(\frac{e^{-2 a\left(x_{I}-x_{F}\right)}}{2 a-c}+\frac{e^{2 a\left(x_{I}-x_{F}\right)}}{-2 a-c}-\frac{e^{-2 i b\left(x_{I}-x_{F}\right)}}{2 i b-c}-\frac{e^{2 i b\left(x_{I}-x_{F}\right)}}{-2 i b-c}\right)\right.} \\
& \left.-e^{-c x_{I}}\left(\frac{1}{2 a-c}+\frac{1}{-2 a-c}-\frac{1}{2 i b-c}-\frac{1}{-2 i b-c}\right)\right]+ \\
\frac{C_{n}^{*} D_{n}}{4} \quad & {\left[e^{-c x_{F}}\left(\frac{e^{-2 a\left(x_{I}-x_{F}\right)}}{2 a-c}+\frac{e^{2 a\left(x_{I}-x_{F}\right)}}{-2 a-c}-\frac{e^{2 i b\left(x_{I}-x_{F}\right)}}{-2 i b-c}-\frac{e^{-2 i b\left(x_{I}-x_{F}\right)}}{2 i b-c}\right)\right.} \\
& \left.-e^{-c x_{I}}\left(\frac{1}{2 a-c}+\frac{1}{-2 a-c}+\frac{1}{-2 i b-c}-\frac{1}{2 i b-c}\right)\right]+ \\
\frac{C_{n} D_{n}^{*}}{4} \quad & {\left[e^{-c x_{F}}\left(\frac{e^{-2 a\left(x_{I}-x_{F}\right)}}{2 a-c}+\frac{e^{2 a\left(x_{I}-x_{F}\right)}}{-2 a-c}-\frac{e^{-2 i b\left(x_{I}-x_{F}\right)}}{2 i b-c}-\frac{e^{2 i b\left(x_{I}-x_{F}\right)}}{-2 i b-c}\right)\right.} \\
& \left.-e^{-c x_{I}}\left(\frac{1}{2 a-c}+\frac{1}{-2 a-c}+\frac{1}{2 i b-c}-\frac{1}{-2 i b-c}\right)\right]+ \\
\frac{\left|D_{n}\right|^{2}}{4} \quad & {\left[e^{-c x_{F}}\left(\frac{e^{-2 a\left(x_{I}-x_{F}\right)}}{2 a-c}+\frac{e^{2 a\left(x_{I}-x_{F}\right)}}{-2 a-c}+\frac{e^{2 i b\left(x_{I}-x_{F}\right)}}{-2 i b-c}+\frac{e^{-2 i b\left(x_{I}-x_{F}\right)}}{2 i b-c}\right)\right.} \\
& \left.-e^{-c x_{I}}\left(\frac{1}{2 a-c}+\frac{1}{-2 a-c}+\frac{1}{-2 i b-c}-\frac{1}{2 i b-c}\right)\right]+ \\
\frac{\left|B_{n}\right|^{2}}{4} \quad & {\left[-e^{-c x_{F}}\left(\frac{e^{2 a\left(x_{F}-1\right)}}{2 a-c}+\frac{e^{-2 a\left(x_{F}-1\right)}}{-2 a-c}-\frac{e^{2 i b\left(x_{F}-1\right)}}{2 i b-c}-\frac{e^{-2 i b\left(x_{F}-1\right)}}{-2 i b-c}\right)\right.} \\
& \left.\left.+e^{-c}\left(\frac{1}{2 a-c}+\frac{1}{-2 a-c}-\frac{1}{2 i b-c}-\frac{1}{-2 i b-c}\right)\right]\right\}
\end{aligned}
$$

Where FV, EV, and WV denotes the full variance, eastern variance, and western variance respectively.

\section{Circulation To The East Of The Barrier}

The following expression is used to calculate the contribution to the circulation of the meridional velocity on the eastern side of the barrier. These calculations are shown on all the contour plots and denoted EC. Note that the time dependence has been omitted since the main interest is how this circulation varies for each spatial mode.

$$
\mathrm{EC}=\int_{d}^{1-d} v_{+}\left(x_{I}\right) d y=\sum_{n=1}^{\infty}\left(-i k \phi_{n}\left(x_{I}\right)+\frac{\partial \phi_{n}\left(x_{I}\right)}{\partial x}\right) \frac{\cos (n \pi d)\left(1-(-1)^{n}\right)}{n \pi}
$$

Due to the $\left(1-(-1)^{n}\right)$ term it is obvious that the modes which are antisymmetric about the mid-latitude and correspond to even Fourier components make a zero contribution to EC. 


\section{Bibliography}

[1] J.K. Atherton. Forced rossby waves in a basin with a meridional barrier and bottom friction. Il Nuovo Cimento, 23, 2000.

[2] J. Pedlosky. A study of the time dependent ocean circulation. Tellus, 22, 1965.

[3] J. Pedlosky. Fluctuating winds and the ocean circulation. Tellus, 19, 1967.

[4] J. Pedlosky and M.A. Spall. Rossby normal modes in basins with barriers. J. Phys. Oceanogr., 29, 1999.

[5] Joseph Pedlosky. Geophysical Fluid Dynamics. Springer-Verlag, 1987.

[6] Joseph Pedlosky. Ocean Circulation Theory. Springer-Verlag, 1996.

[7] J. Pedlosky. The transmission and transformation of baroclinic Rossby waves by topography. J. Phys. Oceanogr., 30, 2000.

[8] J. Pedlosky, L. J. Pratt, M.A. Spall, and K. R. Helfrich. Circulation around islands and ridges. J. Mar. Res., 55, 1997.

[9] J. S. Godfrey. A Sverdrup model of the depth integrated flow for the world ocean allowing for island circulations. Geophys. Astrophys. Fluid Dyn., 45, 1989.

[10] D. B. Chelton, and M.G. Schlax Golbal observations of oceanic Rossby waves. Science, 272, 1996. 